\title{
Engineering English:
}

A multimodal case study of one Engineering and two EAP textbooks

\author{
by
}

Renee Fontenelle

A thesis submitted to the Faculty of Graduate and Postdoctoral Affairs in partial fulfillment of the requirements for the degree of

Master of Arts

in

Applied Linguistics and Discourse Studies

\author{
Carleton University \\ Ottawa, Ontario
}

(C) 2013, Renee Fontenelle 
Library and Archives

Canada

Published Heritage

Branch

395 Wellington Street

Ottawa ON K1A ON4

Canada
Bibliothèque et

Archives Canada

Direction du

Patrimoine de l'édition

395 , rue Wellington

Ottawa ON K1A ON4

Canada
Your file Votre référence

ISBN: 978-0-494-94609-1

Our file Notre référence

ISBN: 978-0-494-94609-1
NOTICE:

The author has granted a nonexclusive license allowing Library and Archives Canada to reproduce, publish, archive, preserve, conserve, communicate to the public by telecommunication or on the Internet, loan, distrbute and sell theses worldwide, for commercial or noncommercial purposes, in microform, paper, electronic and/or any other formats.

The author retains copyright ownership and moral rights in this thesis. Neither the thesis nor substantial extracts from it may be printed or otherwise reproduced without the author's permission.
AVIS:

L'auteur a accordé une licence non exclusive permettant à la Bibliothèque et Archives Canada de reproduire, publier, archiver, sauvegarder, conserver, transmettre au public par télécommunication ou par l'Internet, prêter, distribuer et vendre des thèses partout dans le monde, à des fins commerciales ou autres, sur support microforme, papier, électronique et/ou autres formats.

L'auteur conserve la propriété du droit d'auteur et des droits moraux qui protege cette thèse. $\mathrm{Ni}$ la thèse ni des extraits substantiels de celle-ci ne doivent être imprimés ou autrement reproduits sans son autorisation.
In compliance with the Canadian Privacy Act some supporting forms may have been removed from this thesis.

While these forms may be included in the document page count, their removal does not represent any loss of content from the thesis.
Conformément à la loi canadienne sur la protection de la vie privée, quelques formulaires secondaires ont été enlevés de cette thèse.

Bien que ces formulaires aient inclus dans la pagination, il n'y aura aucun contenu manquant. 


\begin{abstract}
This study is situated within the tradition of systemic functional linguistics, in the field of second language education, and presents a multimodal analysis of three related undergraduatelevel textbooks. The purpose of the research is to determine the veracity of claims made by publishers of English for Academic Purposes (EAP) textbooks that their books enable students to deal effectively with discipline-specific undergraduate textbooks. Two EAP textbooks and one canonical first-year undergraduate textbook, all three for students of Mechanical Engineering, were analyzed in order to provide verbal and visual accounts of meaning-making. Multimodal ensembles, or co-occuring verbal and visual representations, were also analyzed in order to explain how the textbooks generated intersemiotic meanings in their multimodal ensembles. Results show that the undergraduate Engineering textbook employs high levels of technicality, verbally and visually, characterized by complex lexico-grammatical and mathematical choices, and by complex patterns of intersemiotic identification among verbal, visual, and mathematical elements in multimodal ensembles. The EAP textbooks, in contrast, show lexico-grammatical patterns that are consistent with non-technical language, employ simple visual and mathematical structures, and present few multimodal ensembles with few examples of intersemiotic identification. It is suggested that EAP publishers represent the subject matter of Mechanical Engineering in the verbal, visual, mathematical, and intersemiotic patterns that are more consistent with their representation in the discipline's canonical textbooks.
\end{abstract}




\section{Acknowledgements}

I would like to thank both of my supervisors, Dr. Lynne Young and Dr. Graham Smart, for their unique contributions to this thesis-writing process. Lynne, you provided some essential insights and inspirations around which this entire study is framed. Graham, your time and thoughts at every stage of the process contributed as much to my sanity as to this project. I would also like to thank my daughter, Odessa, for her constant patience and for always appreciating another frozen pizza for dinner. 
Chapter One 1

$\begin{array}{ll}\text { Chapter Two } & 6\end{array}$

$\begin{array}{ll}\text { Literature Review } & 6\end{array}$

$\begin{array}{lr}\text { Chapter Three } & 28\end{array}$

$\begin{array}{ll}\text { Theoretical Framework } & 28\end{array}$

$\begin{array}{ll}\text { Methodology } & 36\end{array}$

$\begin{array}{ll}\text { Analytical Framework } & 43\end{array}$

$\begin{array}{ll}\text { Chapter Four } & 53\end{array}$

Verbal Representations of Subject Matter 53

$\begin{array}{ll}\text { Visual Representations of Subject Matter } & 74\end{array}$

$\begin{array}{ll}\text { Intersemiotic Analysis } & 90\end{array}$

$\begin{array}{lr}\text { Chapter Five } & 97\end{array}$

$\begin{array}{ll}\text { Discussion } & 97\end{array}$

$\begin{array}{ll}\text { Implications } & 101\end{array}$

$\begin{array}{ll}\text { References } & 104\end{array}$

$\begin{array}{lr}\text { Appendix A } & 110\end{array}$

$\begin{array}{ll}\text { Appendix B } & 111\end{array}$

$\begin{array}{lr}\text { Appendix C } & 112\end{array}$

$\begin{array}{ll}\text { Appendix D } & 113\end{array}$

$\begin{array}{ll}\text { Appendix E } & 114\end{array}$

$\begin{array}{lr}\text { Appendix F } & 118\end{array}$

$\begin{array}{ll}\text { Appendix G } & 119\end{array}$

$\begin{array}{ll}\text { Appendix H } & 121\end{array}$ 


\section{List of Tables}

Table 1: Oxford process wordings

Table 2: Garnet process wordings

Table 3: Hibbeler: Forces process wordings

Table 4: Hibbeler: Friction process wordings

Table 5: Process summary, by number of clauses per text

Table 6: Nominalization summary, by number of occurrences and wordings

Table 7: Circumstances summary

Table 8: Reference analysis summary

Table 9: Lexical relations summary

Table 10: Oxford representations summary

Table 11: Garnet representation summary

Table 12: Hibbeler: Forces representation summary

Table 13: Hibbeler: Friction representation summary

Table 14: Representation summary, by text

Table 15: Intermodal identification summary, by text 


\section{List of Figures}

Figure 1: Essential components of SFL and relationship to other semiotic theories

Figure 2: A block of uniform weight

Figure 3: Coding orientation summary showing visual scales by text 


\section{List of Abbreviations}

EAP - English for Academic Purposes

ESL - English as a Second Language

ESP - English for Specific Purposes

SFL - systemic functional linguistics 


\section{Chapter One \\ Introduction}

In education, textbooks are almost certainly a part of most people's daily lives. In English as a Second Language education, a materials-driven field, textbooks are ubiquitous and seem to find their way into every aspect of language teaching and learning. Sometimes, we know a textbook is good: it comes highly recommended, students praise it, we know from previous experience that it is an excellent learning resource. Often, though, we may not be so sure. We might turn to the publisher's name, to awards the textbook has won, to the claims the textbook publishers are making when choosing our titles. Sometimes we rely on our intuition in helping us to select a textbook, or on our institution's particular practices. All to say, we are not often in a situation to be able to make an informed, research-driven choice when choosing a textbook. This study presents an investigation into textbooks and identifies factors that may help in making that choice.

The topic of the study presented in this thesis is textbooks, specifically English for Academic Purposes (EAP) textbooks designed for students of Mechanical Engineering. These textbooks are intended to help second-language university or university-bound students develop English skills sufficient to study Mechanical Engineering at the undergraduate level at Englishmedium universities. Publishers of these textbooks make claims that their books will help students access the textbooks and understand the language of Mechanical Engineering. This study is an investigation into those claims.

Hyland and Hamp-Lyons (2002), in the inaugural edition of the Journal of English for Academic Purposes, define EAP "as teaching English with the aim of facilitating learners' study or research in that language" (p. 2). Although this definition is widely quoted, EAP can be de- 
fined, more specifically, as second-language English education designed to help students successfully negotiate the language of higher education. As such, EAP education includes training in study skills, general language skills, and language skills more specific to university settings. EAP programmes are offered through universities, in English-speaking countries and non-Englishspeaking countries, and are normally one year long. Programmes may be either pre-sessional, in which case students complete their EAP study prior to moving into their chosen field of study, or concurrent, studied alongside courses from the student's chosen academic field. For example, here at Carleton, the EAP programme is both pre-sessional and concurrent: it begins as presessional and as students move successfully along, in their final term, they may take a course from their chosen discipline or field of study. Either way, though, if students fail to find success in the EAP programme, entry into the chosen field of study is usually denied. EAP programmes are thus high-stakes endeavours.

In scope, EAP programmes may take a broad approach to content and subject matter or a more focused, narrow approach. In the former, focus is on language that is considered general or common to tertiary education, while in the latter, a programme's focus is directed towards the language of specific fields or disciplines, such as Economics, Health Sciences, or Engineering. This more focused approach is an example of Content-Based Instruction, an approach to language teaching that integrates topics and content from the 'regular' subjects - that is, from Biology, Psychology, Engineering, and so on - within the context of language teaching. It is this approach that is relevant to this current study since the EAP textbooks that I examine are concerned with the subject matter of Mechanical Engineering. Particularly relevant is the issue of how much subject content is enough in language teaching, especially in contexts that find language 
teachers 'teaching' subject content that is well outside their area of expertise. I discuss ContentBased Instruction and the concerns it brings to this study in Chapter Two.

Regarding textbooks, however, few studies exist that look specifically at tertiary-level textbooks, demonstrating Hyland's (2009) observation that "[u]niversity textbooks...are something of a neglected genre" (p. 112). From a materials development perspective, Chapelle (2010), too, recognizes a lack of research, and characterizes the amount of current research as "surprisingly small in view of the impact that materials have in the instructional process" (p. 67). Furthermore, not only are EAP textbooks under-researched, but according to Hyland (2006), "EAP courses still lack a theoretical or research rationale and textbooks too often continue to depend on the writer's experience and intuition rather than on systematic research" (p. 5). Part of the purpose of this study is to fill this gap in research into EAP textbooks.

Publishers of the EAP textbooks examined in this study make claims that their books will help students access the textbooks and language of Mechanical Engineering. Given the highstakes nature of EAP programmes, questions of how much subject content is enough in langauge teaching, and the importance of textbooks in English language education, these publishers' claims need to be investigated as realistic or not. In order to determine how realistic these claims might be, this study will compare the content of two EAP textbooks for Mechanical Engineering with the content of a first-year undergraduate Mechanical Engineering textbook. Since meanings in Mechanical Engineering are expressed visually as well as verbally, this study will analyze both verbal and visual modes of meaning-making. The analysis of these two modes will also lead to an investigation of the relationship between images and language in order to show how these two modes interact to create intersemiotic meanings in multimodal contexts. The study will be 
guided by the over-arching question: How similar is the subject content in two EAP textbooks for Mechanical Engineering students and the subject content of an undergraduate Mechanical Engineering textbook? I have divided this broad question into three smaller questions, more suitable for investigation:

- How is the content in the three textbooks represented verbally?

- How is the content in the textbooks represented visually?

- How do the texts construct intersemiotic meanings in multimodal ensembles?

Systemic functional linguistics (SFL) will provide the theoretical orientation and analytical approach for this study. Briefly, SFL considers the structures of language and their meaningmaking functions as instrinsically connected to the social context in which they are expressed. Furthermore, although SFL itself is concerned with langauge, it also recognizes that language is just one semiotic system among the many that are available in a culture. Thus, SFL has provided the theoretical foundations for the analysis of other non-linguistic semiotic systems, such as the visual mode of expression, as well as for the analysis of multimodality, the co-occurrence of multiple semiotic systems. Therefore, an SFL approach to analysis will allow me to compare and discuss the verbal, visual, and intersemiotic subject content of the textbooks within a coherent framework that will also allow for meaningful comment across those semiotic systems.

Briefly, the results of this study reveal that the two EAP textbooks and the undergraduate Engineering textbook represent the subject matter of Mechanical Engineering very differently. Therefore, this study casts serious doubt on EAP publishers' claims that their textbooks will help students negotiate undergraduate textbooks. This study reveals that the undergraduate Engineering textbook represents Mechanical Engineering as primarily concerned with meanings that con- 
struct the world as inter-connected natural phenomena, events, and doings. Complex verbal, mathematical, and visual expressions realize these meanings. The EAP textbooks construct the world of Mechanical Engineering as concerned with simple phenomena and their definitions, informed by a technical point of view. These representations are realized in simple verbal, mathematical, and visual expressions. An examination of how the texts constructs intersemiotic meanings in multimodal ensembles reveals significant differences in the pedagogic resources that each text mobilizes.

In Chapter Two, I review a range of theories and issues relevant to this study, including discussions of content-based instruction in EAP, principles of visual display and multimodal theory, and multimodal communicative competence in second language education. In Chapter Three, I present the theoretical framework that informs this study, a framework that draws on the inter-related theories of systemic functional linguistics, the social semiotics of visual design, and multimodality. I also describe my methodology before moving on, in Chapter Four, to present my findings which are organized around my three research questions. In Chapter Five, I conclude by reviewing my knowledge claims and then considering the implications of this study, with an emphasis on possible future research directions in teacher education and materials development. 


\section{Chapter Two \\ Literature Review}

In this literature review, I present views from a range of theoretical perspectives of particular relevance to this study. I begin the review by situating this study in its disciplinary context, EAP, then move on to a brief examination of the role of textbooks at the university level and within an EAP context. I then trace the emergence of multimodality as a key issue within the teaching and learning environment, and attempt to broaden the conversation by including relevant theories related to Applied Linguitics. I move on to a closer examination of the role of multimodality in second language teaching and learning before concluding with a discussion of recent theories describing meaning construction in multimodal textual environments.

\section{English for academic purposes: overview and relevant issues}

English for Academic Purposes (EAP) emerged out of English for Specific Purposes (ESP), that field of study overtly concerned with the language of professional or workplace environments. ESP found its beginnings in the 1960 s with a shift in focus from literary English as the object of language study to scientific and technical English (Benesch, 2001). This shift marked an early recognition of two significant reconceptualizations in language education. First, it began the process of re-casting language from a set of grammatical rules that required mastering to a dynamic social resource that changed according to its purpose. Second, it highlighted context, in this case a specific professional context, as a central, extra-linguistic participant in language. While these reconceptualizations would take time to mature, the development of EAP and ESP over the subsequent decades describes the expression of these changes.

Briefly, the beginnings of ESP in the mid 1960 s were primarily expressed through an interest in English for science and technology. The works driving this move were Halliday's newly 
articulated theories on genre and register (Coffin \& Donohue, 2012), theories that demonstrate the "linguistic consequences" of social context (Eggins, 2004, p. 90). The language focus was realized through sentence-level structure and patterns and, at the word-level, through lexical items, and highlighted usage in English scientific texts (Benesch, 2001). Here, then, we see interest in the structural form, not of English generally, but of English situated in a specific context.

By the 1970 s, in an effort to address weaknesses in the work of the 60 s, the earlier grammatical focus was broadened to include rhetorical analysis. Here, explicit connections between linguistic choices and rhetorical purposes became central (Benesch, 2001), connections that exemplified the developing focus of language as dynamic resource concerned with usage as well as form. The 1970s also saw the term English for Academic Purposes come into usage. According to Hyland (2006), English for Academic Purposes was coined in 1974 and first published in 1977 and distinguishes this branch of English language study from the more general English for Specific Purposes. (While much overlapping of terms persists, these distinctions broadly hold true today: ESP is the comprehensive description, EAP the academic, and $E O P$ describes English for Occupational Purposes.)

Trends through the 1980 s again addressed perceived deficiencies from the previous period. This time, interest focused on the development of skills, or specific and desired behaviours that were seen as necessary to success (Dudley-Evans and St. John, 1998; Hyland, 2006). The term skills ended up being interpreted at two levels: "five macro-skills of reading, listening, listening and speaking, speaking, and writing, each consisting of a number of micro-skills" (italics in original, Dudley-Evans and St. John, 1998, p. 75). Micro-skills referred to both linguistic and 
non-linguistic behaviours - for example, learning strategies such as organizing study time or research skills such as referencing journal articles - and might address all macro-skills or just one or two. As Hyland (2006) notes, this focus on skills marked an important shift, a shift away from language towards the learner, "to examining the learners' experiences and to the actual context and situation that they learn in" (p. 18).

Since the late 1980s, EAP has continued to develop although, as Dudley-Evans and St. John (1998) point out, a single approach or movement no longer dominates the field. Instead, a number of influences continue to inform EAP practice today, ranging across practice-based, critical, and analytical perspectives. Two of those influences particularly relevant to this study are discussed below, critical EAP and its focus on the importance of a visual metalanguage and Content-Based Instruction.

Critical EAP is concerned with the socio-political power structures inherent in learning environments, the ways in which academic practices may advantage some and disadvantage others, and, perhaps most importantly, actions in which learners are empowered to recognize, reflect upon, and re-shape aspects of their learning (Benesch, 2001; Hyland, 2006). Researchers recognize that a necessary component of such empowerment is the ability to use a metalanguage with which to articulate thoughts, concerns, and challenges. As Hammond and Macken-Horarik (1999) argue, "systematic discussion of language choices in text construction and the development of meta-language - that is, of functional ways of talking and thinking about languagefacilitates critical analysis" (p. 541). However, as Unsworth (2006) observes, a metalanguage that describes the meaning-making choices of visual constructions and verbal-visual ensembles has not yet been produced. This lack is noticeable, especially given Hyland and Hamp-Lyons' 
(2002) suggestion that EAP learners and teachers are now required to negotiate visual and verbal-visual meaning-making expressions in academic texts as well as the more traditional verbal mode of expression, a point Unsworth (2008) also makes. In fact, Hyland (2009) argues that academic textbooks and articles, with their graphs, photographs, figures, and tables, represent the objective world and reveal how a discipline sees itself and, as a result, a full understanding of written academic discourse can only be achieved by looking at writing and at images. Unsworth (2008) adds to this perspective by drawing attention to the significant support that exists for "the development of a metalanguage that will facilitate metatextual awareness of image/text relations" (p. 385). A verbal-visual metalanguage, then, as well as serving as a tool of critical analysis, can empower learners, reveal how disciplines see the world and themselves, and develop awareness of how images and text work together to create meaning. In recognition of the value of metalanguages, it is hoped that the use of a verbal-visual metalangauge in this study will help to promote the development of a metalanguage appropriate to the discussion of visual and verbal-visual meaning-making.

Belcher (2006) describes Content-Based Instruction (CBI) as "targetlanguage...instruction in subject areas" (p. 138). In other words, CBI is concerned with subject content as presented in second language classrooms, and asserts that content should focus on traditional curriculum subject areas, such as history or biology, rather than on traditional languageoriented topics, such as grammar and vocabulary. The extent to which CBI informs classroom practice can be viewed along a continuum, Lyster and Ballinger (2011) suggest, "varying from 'content-driven programs', such as immersion, to 'language-driven programs', which include language classes either based on thematic units or with frequent use of content for language prac- 
tice" (p. 280). Either way, familiarity with subject matter becomes a central concern for EAP professionals, especially those professionals engaged in programmes geared specifically to introduce second language students to tertiary-level disciplinary study. These programmes prepare students for study in fields as diverse as engineering, economics, or business. However, since most EAP teachers and researchers come from arts and social sciences backgrounds, many lack in-depth knowledge of practices common to the 'hard sciences' (Belcher, 2006) in particular, and to faculties outside of arts and social sciences more generally. Belcher (2006), in addressing the problem of "How much subject knowledge is enough for ESP instructors" (p. 139), discusses several solutions: team-teaching with content area specialists; a re-focusing of the problem that downplays subject matter and highlights problem-solving skills; and the training of EAP profes-

sionals in language study and in a specific content area. (See Lemke, 2002, for his argument that training content area specialists as language specialists is a less time-consuming solution than training language specialists in a scientific content area.) None of these solutions, however, are easy, and questions of how much subject knowledge is enough remain. It is hoped that the results of this study will suggest guidelines to help teachers and researchers determine how much subject content is enough.

\section{The textbook}

A brief review of the historical context of the textbook will deepen understandings of the textbook's purpose. The story of the development of the textbook as instructional genre in the United States, according to Wakefield (1998), arguably begins in the late 1600s with the New England Primer, a textbook designed to develop reading skills through the presentation of religious content. The Primer's overall goal, like other early textbooks, however, was not the devel- 
opment of literacy as an end in itself but to enable access to the Bible (Wakefield, 1998, p. 13). In other words, textbooks such as the New England Primer helped acculturate learners into a specific social community, in this case, a Christian community with its associated Christian beliefs, values, and codes of behaviour. This role of the textbook - to help acculturate into a specific community - has not much changed over the centuries.

One feature of the textbook that has changed over the centuries, though, is the manner in which a textbook accomplishes this goal of acculturation. The early primers described by Wakefield (1998) relied on an already long-established method from, primarily, religious instruction: the catechism style of question and answer (p. 5). Textbooks contained both question and answer, with the answer directly following the question; the role of the learner was to recite the answer correctly upon hearing the teacher read the question. Today, this method of instruction might come across as unduly rote; it does, however, illustrate the textbook's acculturative role. We might understand the significance of this role in light of enduring preferences for the catechism's question and answer format: it remained popular through the next two centuries, with many textbooks written entirely as catechisms (Wakefield, 1998, p. 6). It is still a preferred method today although the format has changed somewhat; today's answers tend to be found in the teacher's book or in the back of the student's book, thus diminishing, perhaps, the sense of indoctrination. By the mid 1800 s, preferences began to shift to textbook formats that worked towards developing student understanding of content material (Wakefield, 1998, p. 11). While catechism formats were not entirely abandoned, other question forms began to emerge. Such questions might require students to refer to personal experience for the answer. Other answers might be found in pieces of expository text or by referring to pictures or diagrams. Regardless of the for- 
mat, echoes of the catechismal style remained: a question was presented in the textbook and textbook users supplied an answer. Since then, time has presented us with our current textbooks, in many ways very much different with full-colour visuals, wide-ranging source material, and all manner of formats, from traditional books to rented digital copies. But in other ways, textbooks remain true to their earlier roots; full of rhetorical questions, they are carefully constructed relayers of knowledge whose main purpose is still to acculturate into a community.

University textbooks. Much like early primers helped enable access to the Bible and the Christian community, undergraduate university textbooks help to enable access into a disciplinary community (Hyland, 2009). In fact, the relationship may sometimes be even closer. Richardson (2004), in his study of undergraduate economics textbooks, observed that "the textbook took on a level of importance and assumed an authority similar to that exercised by devotional texts in the course of religious observance" (p. 510). Given that observation, it is no surprise that Hyland (2009) characterizes textbooks as "indispensable to academic life" (p. 112) and part of "the bread and butter of university life" (p. 96).

Hyland (2009) more specifically describes university textbooks as the systematized recordings of a discipline's significant concepts, accepted theories, current beliefs, and analytical methods. The process of systematizing disciplinary knowledge into textbook form codifies that knowledge and results in, as Brown (1993) first termed it, a "canonizing discourse" (p. 65, see also Hyland, 2009; Richardson, 2004; Swales, 1995). As canonizing discourses, textbooks are powerful: they define reality, realize authority, and are difficult to dispute (Hyland, 2009). While undergraduate students may read that power in a positive light, academic professionals are much less so inclined (Swales, 1995). According to Swales (1995), a range of factors contribute to the 
textbook's lowly status: textbooks compile widely accepted beliefs instead of promoting new research; textbook writing, in offering financial rewards, promotes commercialism over scholarship; textbooks transfer information instead of generating knowledge. Nonetheless, both Hyland (2009) and Swales (1995) see potential in textbooks. Hyland (2009) reminds us that "learning a disciplinary culture and learning its language are inseparable" (p. 121) and that textbooks, as written examples of how a discipline construes itself and one of the primary means of student interaction with the discipline, provide a key opportunity for students to engage with and learn the language of that discipline. Swales (1995) takes another approach and finds potential in textbooks through addressing their perceived weaknesses. Accordingly, textbooks could be improved by incorporating current research, 'owning up' to theoretical or other uncertainties, adopting a more dialogic style, and so on. In this 1995 article, Swales seems to re-evaluate the textbook more positively and apologizes for omitting textbooks in his seminal Genre Analysis.

ESP/EAP textbooks. Swales' concerns about textbooks in 1995 were not his first. As early as 1980, in "ESP: The Textbook Problem", he declared that "ESP textbooks have been in many respects an educational failure" (p. 11). His key argument concerned the value of locallyproduced materials over commercially-produced textbooks. Swales, perhaps surprisingly given the article's title, generally comes out of this article in favour of textbooks, albeit with provisos, and determines that commercial textbooks have a specific role in addressing more universal aspects of language education. Locally-produced materials, on the other hand, find their role by filling in culture-specific gaps. This long-standing argument continues to speak to core issues in the field, especially given the field's materials-driven approach. As recently as 2005, Harwood asked "What Do We Want EAP Teaching Materials For?"and identifies similar positions as 
Swales did in 1980 . The strong anti-textbook position identified by Harwood holds that no commercially produced materials should be used while the weak argument holds that although commercially produced materials are often unsatisfactory, they need to be improved upon rather than disposed of. Little argument, however, exists in the approach to textbook improvement. All agree that research is the key (Harwood, 2005; Hyland, 2006; Swales, 1980), and Swales has been saying so since 1980, at least. Swales (1980) also claims that challenging textbook content is important since such challenges initiate textbook revision and change. It is in this context that this study, at least partly, finds its rationale.

\section{Emergence and role of multimodality in communication and learning}

As early as the mid 1990 s, the expanding role of visuals in education and other areas of communication made it apparent that traditional linguistic frameworks of understanding and analysis were no longer sufficient. In 1994, O'Toole became the first researcher within Applied Linguistics to theorize the visual mode of expression with his publication The Language of Displayed Art. This work, derived from Michael Halliday's systemic functional linguistics (which I review in my discussion of theory, below), describes painting, sculpture, and architecture within a social semiotic framework that sees meaning-making and social context as completely connected. Two years later, Kress and van Leeuwen (1996) followed suit with the publication of their highly influential Reading Images: The Grammar of Visual Design (with a reworked second edition published in 2006), also based on Halliday's linguistics and within a social semiotic framework. However, while these visual theories gave rise to a metalanguage with which to discuss the visual mode, analytical and theoretical approaches remained largely monomodal. Kress and van Leeuwen (2001) themselves, in commenting on Reading Images, admit to being "spe- 
cialists' of the image" and briefly document the Western world's "distinct preference for monomodality" (p. 1). The 2001 book from which this quote is taken, Multimodal Discourse: The Modes and Media of Contemporary Communication, marks Kress and van Leeuwen's transition away from the monomodal and into the multimodal, as it explores and theorizes multimodal communication within a social semiotic framework, and explains the array of meaning-making modes now part of commercial, social, professional, and educational spheres of life.

An important effect of this movement towards visualization and multimodality in education can be seen in calls for new definitions of literacy (see, for example, Bezemer and Kress, 2008, 2009; Hyland, 2006; Jewitt, 2008; Kress \& van Leeuwen, 2001; New London Group, 1996; Unsworth, 2008). By 2000, Kress was arguing that "It is now impossible to make sense of texts, even of their linguistic parts alone, without having a clear idea of what these other features might be contributing to the meaning of a text" (p. 337). From a perspective informed by education, the New London Group in 1996 was already recognizing the necessity of understanding these 'other features', and thus introduced the term 'multiliteracies' to acknowledge the role that non-linguistic features played in meaning-making. The term is part of an expansive model of literacy and learning that, among other things, calls for a re-definition of literacy to include "forms that are becoming increasingly significant in the overall communications environment, such as visual images and their relationship to the written word" (New London Group, 1996, p. 61). By the early 2000 s, many of applied linguistics' leading scholars were acknowledging the critical role of understanding visual and multimodal resources in the negotiation of academic texts (Hyland, 2009; Kress, 2000; Kress \& Burn, 2005; Lemke, 2002, van Leeuwen, 2005). Lemke's (2002) quote sums up overall views effectively: "The narrowest definition of literacy would fo- 
cus solely on verbal literacy with written media, but this definition is intellectually untenable today" (p. 22). Jewitt (2008) clarifies an important dimension of this argument by pointing out that the way knowledge is represented - the modal form it takes, whether written, spoken, visualized, or presented in some other semiotic system - plays a crucial role in knowledge construction. The form of knowledge representation is thus foregrounded because it is integral to meaning. Jewitt (2008) argues that explorations of the modes of representation that are used in classrooms is essential to an understanding of modern teaching and learning.

One of the consequences of increases in non-verbal meanings within education translates into changed responsibilities for teachers. Within education generally, practitioners are "increasingly required to understand and translate the progressively more complex interactions between verbal and non-verbal features of academic texts" (Hyland \& Hamp-Lyon, 2002, p. 8). Hyland and Hamp-Lyon (2002) go on to observe that these new complexities require further research in order to gauge their impact and to fully understand their role within EAP. One of the difficulties inherent in incorporating visual and multimodal meanings into EAP is EAP's identity as language-oriented. This identification is, naturally, strong, giving Kress (2000) cause to observe that English language professionals act as if "language fully represented the meanings they wish to encode and communicate" (p. 337). Overall, then, the movement towards increased multimodality in communication seems to be more fully articulated theoretically than understood in terms of practice, particularly within EAP, which understandably has such close ties to language. A number of studies from outside Applied Linguistics are also investigating the use of multimodal resources and their effects on learning. Results from a range of fields are remarkably 
similar, and I present them here to provide insights into broader educational practices that may inform EAP, especially given its current struggles with the boundaries of language.

Mayer $(2005,2008)$, a leading scholar in psychology, has conducted numerous studies on college-age students that investigate the role of multimedia in their learning ('multimedia' is Mayer's term, more or less equivalent to the term 'multimodal' that I use in this study). Of particular interest are experiments that ask students to read expository messages presented in multimodal learning materials - such as written text with corresponding diagrams presented on a following page, or written text with diagrams integrated into the corresponding writing - and then test the students on their learning of the content thus presented. Over 15 years of studies like these have produced results consistent enough that Mayer has been able to develop a set of principles to explicate multimedia message design that enhances learning (Mayer, 2008). Of the ten principles he enumerates, the following five relate to written/visual messages:

- Coherence principle: omit unnecessary words or pictures

- Signaling principle: highlight essential information

- Spatial contiguity principle: place related words and pictures closely together

- Segmenting principle: break information into small parts

- $\quad$ Retraining principle: explain operation of parts first

Mayer's 'Spatial contiguity principle', emphasizing the importance of placing related words and images together, is based on the rationale that learners build mental connections between words and images. If they must search for corresponding images, or words, cognitive capacity is wasted during the search thus reducing learners' ability to construct mental connections. Tufte (2006), a prominent researcher in political science and statistics who is noted for his writ- 
ings on information design, also formulates a set of principles of design. Although Tufte's principles focus on analytical data rather than multimodal design, one principle that is strongly consistent with Mayer's spatial contiguity principle, and asserts the "Integration of Evidence" (p. 122). This principle explains that words, numbers, images, and diagrams should be completely integrated with one another. According to Tufte (2006), the purpose of this integration and "the essential test of text/image relations is how well they assist understanding of the content" which is accomplished through an understanding of "what the words mean in their relation to the images, and what the images mean in relation to the words" (p. 88, italics in original). Both Mayer's and Tufte's integration principles are consistent with multimodal theorizing that resources from all modes inter-relate to produce meanings.

Atkinson (2005) adds another dimension to the importance of modal inter-relation with reports on studies investigating the integration of language and mathematics in both static (print) and dynamic (spoken or moving image) deliveries. Relevant to this current study is research concerned with printed mathematical graphics and written text. Students were presented with one of two geometrical mathematics problems: either problems in which written language, embedded with mathematical expressions, was integrated with diagrammatic examples worked out to show the solution and how it was achieved, or problems in which worked-out diagrammatic examples were not integrated with the language and mathematics. Consistently, students receiving the integrated problems were able to solve problems more accurately and quickly than students who received the non-integrated problems. While these studies are limited in that all worked exclusively with geometry problems, the implications are consistent with Tufte and Mayer. That is, the studies described above - mathematics studies, studies from a visual perspective, and studies 
with expository material - all show that integrated formats of textual instruction foster learning and improve performance. These implications are also significant from a multimodal perspective, in the sense that they could provide guidelines in determining verbal - visual balances, particularly in EAP, where struggles with these meanings and balances are ongoing.

A different perspective on the visual and multimodal is brought forward by Olive and Passerault (2012), from the field of cognitive psychology. Citing a range of studies and theoretical articles, the authors suggest that reading and writing should be considered visuospatial activities, activities guided by the eye and graphic traces on the page. Writers and readers use a text's visuospatial representations to find and retrieve information. This visuospatial dimension is also engaged in the construction of mental representations of text during the reading and writing processes. Olive and Passerault (2012) cite additional studies that suggest these mental representations may be "multidimensional" (p. 334), integrating semantic, verbal, and visuospatial content within an episodic memory format. Furthermore, concrete and abstract content may be processed differently. Experimental evidence, although limited, seems to suggest that the processing of concrete content and abstract or figurative content is associated with different parts of working memory and thus processed differently. These claims are relevant to my present study in two ways. First, since visuospatial representations seem to play a role in how readers and writers locate and retrieve written verbal information, visuospatial representations would ideally be developed similarly in the EAP textbooks and in the undergraduate textbook. Visuospatial representations are of particular consequence in this present study since the books in this study are textbooks, in other words, books to which students turn specifically to find and retrieve information. Second, studies also suggest that concrete and abstract information are processed differently. 
Given that, it might be important that information in the EAP textbooks is presented with similar levels of abstraction and representation as information presented in discipline-specific textbooks. These points will be addressed again in the Chapter 5 .

Multimodality and second language learning. A common observation holds that visual and other non-linguistic meanings aid in the comprehension of a second language. Lemke (2002), in addressing this observation, contends that the value of visual and non-linguistic elements in aiding comprehension is determined by two factors. First, visuals, mathematical symbols, and other meaning systems can only aid understanding of a second language if the meanings represented in the non-linguistic systems are already universal in their interpretation. In other words, mathematical symbols will only help in understanding English text, for instance, if the mathematical symbols mean the same across all languages (or, at least, across English and the native language of the English learner reading that text). Second, even when non-linguistic meanings are universal, as they are in much of mathematics, for example, language users still must know how semiotic systems are integrated to produce a unified text. English is just beginning to discover and articulate the logic and conventions that prescribe how meanings from different semiotic systems are woven together. From a language learner's perspective, a knowledge of how semiotic systems are integrated is crucial to comprehension, particularly given the complex nature of multimodal literacy (Royce, 2007).

In comments directed towards language learning and science, Lemke (2002) observes that science relies on a number of semiotic systems in making meaning, including language, mathematics, graphs, diagrams and so on. He points out that language, or any one semiotic system that science uses, cannot be separated out of a text with the result that meanings remain in- 
tact. Attempts to focus exclusively on language as the means to understand scientific concepts cannot work since science means with a multiplicity of semiotic resources. Each semiotic system needs to be comprehended, as does the integration of those systems in the production of scientific meaning. It is reasonable to assume that Engineering, similar to science in its use of multiple semiotic systems to communicate meaning, must also be understood in similar terms.

Royce $(2002,2007)$ puts forward the concept of 'multimodal communicative competence' for second language learners in recognition of the increasing role that non-linguistic semiotic systems play in communication. Communicative competence, a defining concept in the field of second language learning, was first put forward by Hymes in 1972 and, in brief, relates to the language learner's need to know how language is used in social life in order to communicate effectively in a second language. This concept is generally seen to contrast with grammatical approaches to language learning in which students are encouraged to learn and memorize grammar rules. Royce extends notions of the communicative concept because "it is not enough, to meet students' needs, to focus only on language; teachers should begin to focus on and develop students' abilities in visual literacy" (p. 366). The approach Royce envisions starts with a recognition that images are 'culturally bound', and the meanings expressed by an image in one culture may not be the same meanings as those expressed by the same image in another culture (p. 366). Like Lemke (2002), Royce also acknowledges that the inter-relation of modes, how language and image work together, for instance, is critical to understanding and that this inter-relationship may also be culturally sensitive. Royce sees approaches to second language education that incorporate these multimodal meanings as providing entrance to the target culture, since the ways that people in the target culture combine modes to produce meaning would offer insight into that culture's 
practices and ideologies. Since Royce's comments are in context of second language learning, it would be safe to assume that she refers to language cultures in her discussion. Yet much in the same way that a linguistic culture, English speakers, for example, might be understood in terms of how it communicates, a professional or disciplinary culture might be understood in the same way.

\section{Intersemiosis: meaning construction through text-image relationships}

Intersemiosis is a multimodal concept and refers to the semantic relationships developed between language and image that "show how the visual and verbal modes interact to construct the integrated meanings of multimodal texts" (Unsworth \& Cléirigh, 2009, p. 151). While intersemiosis has generated some interest in different research traditions, including Mayer's psychology studies, discussed above in context of his multimedia principles, and Roth, Pozzer-Ardenghi, and Han's (2005) study of Brazilian school science textbooks, these studies fall outside of a semiotic framework so will not be reviewed here. Instead, I focus on those studies informed by social semiotic approaches to meaning, which derive from Halliday's work in SFL.

Royce $(2002,2007)$ developed the framework of intersemiotic complementarity around concepts of lexical cohesion, a lexical resource part of the SFL framework of analysis. Lexical cohesion describes relationships among a text's content words, and Royce applies this concept to describe links between language and image. Relationships or links of repetition, synonymy, antonymy, meronymy (relationships of part/whole, for example, page/book), hyponymy (relationships of class/sub-class, for example, book/textbook), and collocation describe how visual elements and language elements, which are co-present in a text, are connected. In her studies of a high school textbook (2002) and an article in the Economist (2007), Royce found that most 
image-text relationships were built around relationships of repetition, meronymy, or collocation. The meronymy and collocation relationships demonstrate that visual-verbal meaning can be, according to Royce (2007), "'synergistic' in nature, a concept which describes the ability of elements, in the act of combining, to produce a total effect that is greater than the sum of the individual elements" (p. 103).

Martinec and Salway (2005) introduced another way of looking at image-text relations, also based on the systemic functional work of Halliday, and took the concept that describes the types of relationships between clauses and applied it to the relationships between language and image. This concept has two components, a structural component and a semantic one. Structurally, relationships between clauses are either equal and co-ordinate, or unequal, in which case one clause is independent and the other is dependent. Semantically, relationships between clauses can be one of three types. First, in elaboration, the meaning of one clause is restated in the other; in extension, the meaning of one clause is extended or added to the other; and in enhancement, the meaning of one clause is qualified in the other clause. Martinec and Salway (2005) extend these structural and semantic components to describe relationships between language and images. The structural component describes the relationship between image and language as equal or as unequal, and, if unequal, the image as dependent on the language, or the language as dependent on the image. The semantic component describes the image-text relationship in terms of elaboration, extension, or enhancement. As Unsworth and Clérigh (2009) observe, though, a number of problems exist with this system. The most problematic area addresses the value of determining equal or unequal status between language and image, and prompts Unsworth and Clérigh to ask "How does it further our understanding of how language and image collaborate in the 
creation of a unified, coherent text?" (p. 153). Further, Unsworth and Cléirigh (2009) argue that this system does not account for the reciprocity, or the flow of meaning from text to image and from image to text, that exists between image-text relations. This reciprocity can be seen, for example, in a caption that explains its accompanying image and in the image when it provides additional information or detail not explicated in its caption.

Kress $(2000,2010)$ and Kress and van Leeuwen (2001) approach the issue of intersemiosis by considering the affordances inherent to each mode (a topic which I explore further in my discussion of multimodal theory in Chapter 3). As Kress explains (2000):

The semiotic modes of writing and of image are distinct in what they permit, that is, in their affordances. Image is founded on the logic of display in space; writing (and speech even more so) is founded on the logic of succession in time. Image is spatial and nonsequential; writing and speech are temporal and sequential. That is a profound difference... one of its effects is a functional specialization of speech, writing, and image in which each is used to do that for which it is best suited. (italics in original, p. 339)

Lemke (1998) describes a similar distinction between the kinds of meanings that language is better suited to make and meanings that images are better suited to make, and characterizes linguistic meanings as typologically oriented and visual meanings as topologically oriented. Bezemer and Kress (2008), in their diachronic study of high school textbooks, develop the idea of modal affordances into epistemological commitments, or the unavoidable affordances associated with using a particular mode. One of the epistemological commitments recognized by 
Bezemer and Kress in the textbooks they examined was the gain in generality allowed for by writing and, conversely, the gain in specificity that images permit.

Unsworth and Cléirigh (2009) focus on the idea that a visual and a verbal representation of the same thing or phenomenon simultaneously identifies and differentiates that phenomenon. More simply, when a visual and a verbal representation refer to the same object or phenomenon, different aspects of that object are foregrounded, in accordance with the affordances offered by the mode. Following this thinking, they apply the grammatical concept of relational identification, from systemic functional linguistics, to explain image-text relations. While I explain this approach to visual-verbal analysis more thoroughly in Chapter 3, as it is the analysis I use in the present study, I outline the basic concepts here. Briefly, certain verbs describe relationships of mutual identification. These verbs, or in systemic functional terms relational identifying processes, are most commonly be or have, and a typical example might be She is the leader. The point to note is that both participants in the clause, she and the leader, refer to the same entity. Each participant also has a functional role: she is the Token or the holder of the identifying Value, which, in this case, is the leader. While each participant refers to the same entity, there is no redundancy of meaning. In much the same way, Unsworth and Cléirigh (2009) see the relationship between images and text as one of mutual identification: the images identify the text, and the text identifies the images, with each mode highlighting or foregrounding different meaning or information. Unsworth and Cléirigh see this theory, referred to as intermodal identification, as addressing inconsistencies or problems with the previous theorizing of image-text relations. That is, intermodal identification does not rest on determining which representation, verbal or visual, is independent and which subordinate. Rather, intermodal identification accounts for 
the differing affordances each mode offers. Unsworth and Cléirigh (2009) also consider pedagogic functions of intermodal identification. They point out that, in a text, if language is present without an accompanying image, the reader must supply an image from his/her prior experience of the language, and conversely, if an unfamiliar image is presented without a verbal gloss, the reader must supply the wordings from his/her prior experience of the image. However, if the reader has limited prior experience of the language or the image, the language cannot be visually encoded nor can the image be verbally glossed. This observation is important in context of second language learners, particularly those learning the meanings of a highly visual discipline such as engineering. As Unsworth and Cleirigh go on to point out "[t]he more exotic/technical/abstract the field, the more important the intermodal identification pedagogically" (p. 160).

\section{Literature review summary}

In this literature review, I have brought together views and influences from perspectives that are relevant to the present study. Of the current influences in EAP, I discussed the importance of developing a visual metalanguage in order to negotiate visualized texts, to further understand how a discipline sees itself, and to recognize how images and texts together create meaning. I also highlighted content-based instruction issues that ask how much subject content in a language classroom is enough. It is my hope that this present study will provide useful insights to help determine appropriate subject content levels. In my discussion about textbooks, I brought forward calls for research to influence the content and production of EAP textbooks, a call that this study addresses. The longer discussion on multimodality in education and second language learning highlighted a number of key issues. First, the movement from literacy to multiliteracies necessitates an understanding and exploration of the multiple modes of representation in educa- 
tion. I then briefly provided understandings developed in cognitive science that emphasize the importance of integrating verbal, visual, and mathematical resources in learning materials. I also presented a study that suggests the manner in which information is presented in its print form is crucially important in developing and using visuospatial representations that help with finding and retrieving information. Both of these concepts I will return to in my Discussion section, to see whether integration and visuospatial differences exist in the EAP and engineering textbooks that I analyze. My discussion of multimodality and second language learning underlined the importance of integrating verbal, visual, and mathematical meanings and highlighted the necessity for second language learners to understand how multimodal integration works. This present study aims to explicate how verbal and visual meanings are integrated in the two EAP textbooks and in the engineering textbook. I then referred to Royce's $(2002,2007)$ concept of multimodal communicative competence and her view that this competence helps provide entrance into the target culture, a view I extend to include entrance into a disciplinary culture, and which, by inference, I suggest will help learners enter engineering, especially given engineering's visual practices. I concluded the literature review by tracing recent thought on the image-text relations of intersemiosis and paid particular attention to the theorizing of Unsworth and Cléirigh (2009) since I will be applying their system of analysis to the intersemiotic analysis in this present study. 


\section{Chapter Three \\ Theoretical and Methodological Framework}

This chapter is divided into three sections: the first section presents the theoretical background that orients this study, the second section describes the methodology, while the third section presents the analytical framework and provides details of each of the analyses I employed.

\section{Theoretical Framework}

In order to understand how the three textbooks - two EAP textbooks and one first-year Engineering textbook - represent their subject content and create multimodal ensembles, I draw on three inter-related theories: systemic functional linguistics to describe linguistic representations, social semiotic visual theory to describe visual representations, and multimodal theory to bring together the linguistic and visual. I discuss how each theory is rooted in systemic functional linguistics and provide a rationale for selecting inter-related theories for this analysis.

\section{Systemic functional linguistics}

Systemic functional linguistics (SFL) is both a theory of language and a framework of analysis developed by the influential British linguist Michael Halliday (1985/2004). This theory sees language as one of many meaning-making resources all of which are intrinsically embedded in the social environment. In brief, socially situated language users exchange meanings, spoken and written, as part of the process of accomplishing and carrying out daily life. As a meaningmaking resource, language is a semiotic system; as a system that is expressed and interpreted within a sociocultural context, that semiotic is inherently social since meanings are exchanged in social contexts. Hence, the SFL perspective that sees language as a social semiotic takes as fundamental these two core concepts. 
The theory of SFL explains that language is organized as a system of choices. In other words, using language is not a matter of choosing a word or a series of words from the pool of all words. SFL asserts that language is available to users through complex systems of choice. These systems limit the available choices such that in a given context a language user is no longer selecting from the entire resource and instead selects from a particular range of choices. Choices are made from within a set of options that have, themselves, been specified by choices made previously. For example, the selection of language feature $a$ means that the next choice will be either $x$ or $y$. At each point of choice, a system that defines possible alternatives becomes available so that the language user is making a continual series of increasingly delicate or detailed choices throughout the communication process.

SFL is functional and describes its functionality in two ways. First, according to Halliday (1985/2004), functional grammar "is designed to account for how language is used" (bold in original, p. xii). Every use of language occurs in a particular context, to meet particular purposes and needs of the person using the language. Functional grammar accounts for context and explains language in terms of the purposes and needs of each context. Second, since language is essentially a meaning-making resource, the meaning that language carries is described in functional terms. SFL theory calls these meaning components 'metafunctions' and identifies three general purposes and meanings that account for all uses of language. The first metafunction, the ideational, expresses content and experience of the real world and is thus the component of the most interest in this study. The second metafunction, the interpersonal, expresses inter-relational meaning between language users. The third metafunction, the textual, expresses the organizational meanings that structure ideational and interpersonal content. Importantly, all of the meta- 
functions are realized simultaneously; that is, a language user constructs ideational, interpersonal, and textual meanings simultaneously with each utterance. Each metafunction is also organized around one or more of the previously-described systems. For example, within the ideational metafunction, the system of transitivity describes the processes, or the actions and doings, of the real world. Processes are realized at the lexico-grammatical level, the level of wordings plus structures. In this study, linguistic analysis occurs at the lexico-grammatical level.

One of Halliday's (1985/2004) purposes in constructing systemic functional grammar was to "make it possible to say sensible and useful things about any text" (p. xv). Since this purpose more or less describes the aim of my study in that I want to say sensible and useful things in the comparison of the two sets of texts I analyze here, SFL becomes a natural analytic framework. A systemic functional approach examines the functions to which language use is directed and thus permits a focus on experiential meanings, those meanings that encode the comings and goings of people, places, and things in the real world. These functions are realized through the lexico-grammar, the analysis of which furnishes a linguistic description of the structures and wordings that realize the experiential meanings. This functional level of analysis means that comparisons among texts occurs along meaning-making dimensions, and the expression of these meanings through the lexico-grammar permits patterns of difference and similarity to be noted and explained. Furthermore, the systemic use of language emphasized in SFL theory, the networks of choice set up by previous choices, allows for straightforward comparison of texts. It is plain to see which texts establish which systems and which choices are selected within each system. 


\section{Social semiotic visual theory}

According to Iedema (2003), four significant insights distinguish SFL and provide space for social semiotic theory to flourish. These four insights, already touched on above, are as follows: first, the conceptualization of meaning as functional components, namely, the ideational, the interpersonal, and the textual; second, the formulation of meaning-making at phonological or orthographical, lexico-grammatical, and semantic levels; third, the notion that language expresses context, specifically, the topic at hand, the type of relationship between the language users, and the role of language in the interaction (more formally referred to as field, tenor, and mode); and fourth, a movement from a focus on the sentence to a focus on text as a mode of social action. These insights enabled a shift in analysis that, according to Iedema (2003) "did justice to texts' socially meaningful role" (p. 31). By the early 1990s, Martin had devised methods of SFL text analysis that linked language features to ideology and worldview. This call was taken up by Fairclough in the late 1980s and mid 1990s with his Language and Power (1989/2002) and Critical Discourse Analysis: The Critical Study of Language (1995/2010), both of which looked at the ways social and political power are expressed in text. Up to this point, however, most analytical work was still language centered. But the mid and late 1990 s saw movement into semiotic realms far removed from language, including O'Toole's application of SFL to painting and visual art in 1994, van Leeuwen's use of SFL in the analysis of sound and music in 1999, and Kress and van Leeuwen's application of SFL to visual images in 1996. See Figure 1, below, for a visualization of the relationship of SFL to the other semiotic theories drawn from it. 

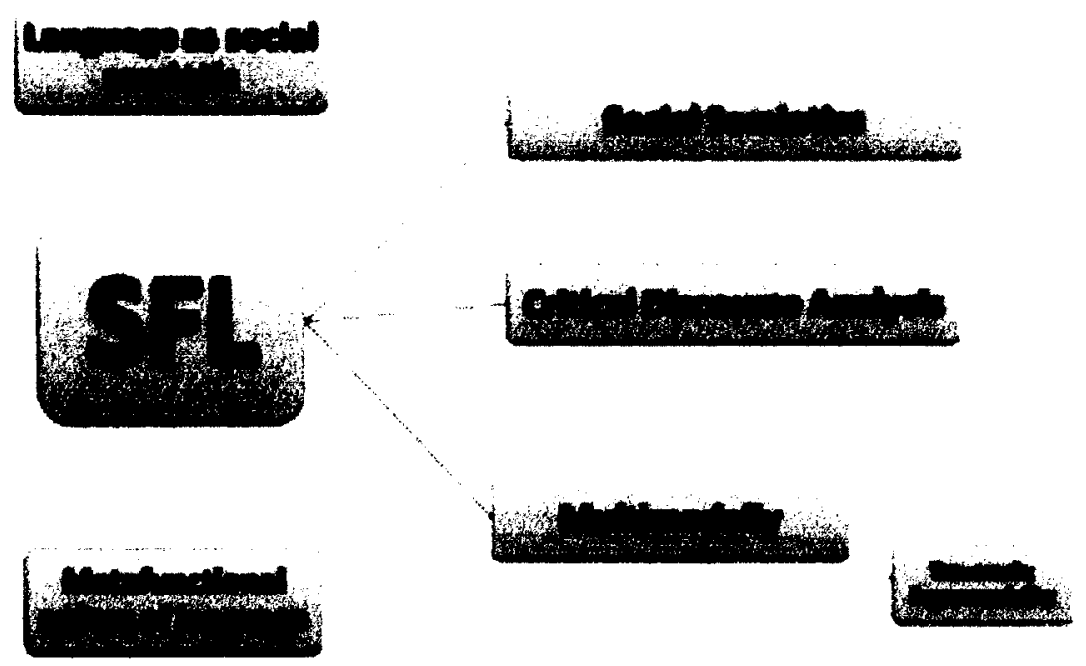

Figure 1: Essential components of SFL and relationship to other semiotic theories. Adapted from L. Young.

Kress and van Leeuwen's (1996) landmark Reading Images: The Grammar of Visual Design explicates a visual grammar based on the social semiotic theory outlined by Halliday in his description of language. The theory proposes that visuals, in much the same way as language, constitute a meaning-making resource. The meaning expressed through any visual entity, like the meaning in a word or phrase, is socially negotiated; meanings are understood in a sociocultural context. A shape, for example a circle, carries a particular meaning because people using that shape in a specified context tacitly and repeatedly agree to its meaning.

This theory also articulates networks of systems and the three over-arching metafunctions that describe meaning. Kress and van Leeuwen re-name the metafunctions - as the representational, the interactive, and the organizational - to reflect the modified purpose of each in the visual mode, but the core meanings remain the same. The representational metafunction relates a visual experience or interpretation of the world while the interactive metafunction describes relationships between the producer of the visual and its viewer and between the represented entities. 
The organizational metafunction describes compositional resources that show how parts work together to form a whole. As in SFL theory, each of the metafunctions is expressed through systems which offer the user a set of choices at various points in the communication process. So, for example, the representational metafunction, the metafuntion of concern in this study, offers an initial choice between representing an aspect of reality as a narrative, or active, process or as a conceptual, or being, process. As meaning is further articulated, additional sets of choices are enacted, with each choice in the process expressing meaning in all three functional terms simultaneously.

One of the obvious advantages in selecting this visual theory as an approach to the analysis of visual elements is its complementarity with SFL. Since both theories share fundamental concepts and are articulated in similar manners, they provide analyses that can be interpreted using similar understandings and terminology. For example, linguistic patterns of transitivity that express actions and participants in the real world can be discerned in connection with visual patterns of representation that express visual participants and actions. In this way, comparisons across modes can be made. Such comparisons can be helpful in revealing patterns of representation that are consistent across semiotic modes rather than explaining the texts as multiple monomodal constructions. Since each of the analyzed texts is meant to be perceived as a unified multimodal whole, it makes sense to acknowledge that unity in the analysis process, when possible.

\section{Multimodal theory}

While social semiotic theory was being taken in a more politicized critical direction by Fairclough in the mid 1990 s, it was also moving in more varied directions, to describe discrete meaning-making resource systems, such as painting, theatre, and visual design. It was out of 
Kress and van Leeuwen's work around this time, according to Iedema (2003), that the term 'multimodality' emerged. While Jewitt (2008) identifies 1996 as the year of multimodality's inception, she points to its emergence as a "response to the changing social and semiotic landscape" (p. 246). The changes to which she refers are what Iedema (2003) calls " $[t]$ he increased ubiquity of sound, image, film, through TV, the computer, and the internet" (p. 33). Although our western culture has been dominated by monomodal meanings, namely meanings made through language, these recent changes in technology have foregrounded other meaning-making modes such that a monomodal perspective on meaning-making is no longer possible. The term multimodality, then, highlights the importance of taking into account multiple semiotic systems, in recognition that meaning is made through the use of many resources, not just one. In fact, the multimodal approach asserts that all meaning-making is multimodal. For example, speech is multimodal in that in relies on at least two modes to communicate meaning: the verbal mode and the aural mode. Other modes may provide additional meanings, such as meanings supplied by gesture, music, images, and so on.

This appreciation of multimodal meaning-making has also meant a movement towards views that de-centre language as the preferred meaning-making resource (Iedema, 2003; Jewitt, 2008; Kress, Charalampos, Jewitt, \& Ogborn, 2001; Kress \& van Leeuwen, 2001). Language, instead of taking centre stage in the communication process, is seen as one mode among many that are used to make meaning. Sometimes, the verbal mode is foregrounded, but often other modes are as significant, or more so, in many meaning-making processes. Importantly, however, is the view that all modes used in communication create meaning. 
Kress and van Leeuwen (2001), in their outline of multimodal theory in Multimodal Discourse: The Modes and Media of Contemporary Communication, put forth two key concepts, both of which inform this present study. First, the notion of modal 'affordances' or 'epistemological commitments' (more thoroughly discussed in Kress, 2010) describes the unavoidable decisions that must be made when using a semiotic resource. For example, a drawing must show spatial relationships; it is not possible to visually represent an object without showing some sort of spatial relatedness among the object's parts. In a second example, language makes a commitment to linear, temporal order; sentences unfold sequentially, and it matters in which order they unfold. A closely related concept holds that meanings that can be communicated in one mode cannot all be communicated in another mode (Saint-Martin, 1994; Lemke, 1998; Kress, 2000; Unsworth, 2006). For example, Saint-Martin (1994) notes that the visual mode "is formed by dense and continuous elements... oriented in all directions in a multidimensional context, reversible and superimposable" while verbal language, in contrast, "is linear, unidimensional, discontinuous, sequential... based on discrete elements (words) separated by voids" (p. 387). Lemke (1998) characterizes these two types of meaning as the typological meanings inherent to language and the topological meanings of visual representation, and notes that science utilizes the visual mode to express topological meanings that are difficult to communicate linguistically. These examples lead into the second key concept informing this study: in multimodal ensembles, each mode adds meaning to the ensemble, and additional modes of expression always add meaning. As Jewitt (2008) observes, "all modes... contribute to the construction of meaning in different ways. Therefore, no one mode stands alone in the process of making meaning; rather, each plays a discrete role in the whole" (p. 247). Lemke (2002) rather smartly explains that semiotic 
resources are only separable analytically, that all meaning is produced in the integration of semiotic resources. These concepts are integral to this study as it is my contention that in a discipline as visually oriented as engineering, an account of only, or primarily, the verbal meanings does not explain how engineering means.

\section{Methodology}

In this section, I describe the methodology of the study. The description includes the textbook selection process and rationale, the rationale behind the selection of data from each textbook, and the approach taken to analyze the data.

This study is a qualitative discourse analysis of three undergraduate textbooks that I undertook in order to investigate claims made by publishers of English for Academic Purposes textbooks designed specifically for engineering students that their books prepare students to access discipline-specific textbooks and language. I chose to focus on EAP textbooks for engineering students for three reasons. First, I was interested in exploring the relationship between written language and visual images in language teaching materials. Since engineering is a discipline that relies strongly on visual practices, and many of its textbooks reflect that concern, the examination of this discipline seemed relevant. Furthermore, since engineering is also attracting increasingly large numbers of non-English speakers and, consequently, the teaching of engineering-bound students is becoming a greater part of the activity of English language instructors, an informed comprehension of the significant role that a textbook plays in an undergraduate's life seemed a meaningful pursuit. Third, as a Faculty of Arts and Social Sciences (FASS) graduate, I had so little experience of engineering that I was unable to ascertain the value of an EAP publisher's claim. Since most English language teachers are similarly educated, I 
thought it a benefit to learn more about a discipline within which we are so likely to encounter our students and our teaching.

\section{Textbook selection}

I began by choosing the EAP textbooks, looking specifically for those textbooks concerned with engineering; I found two, one from Oxford University Press and the other from Garnet Publishing. Since the Oxford textbook focused on electrical and mechanical engineering but the Garnet book covered only Mechanical Engineering, I decided to focus on Mechanical Engineering. I then conducted research to find the names of important undergraduate textbooks in Mechanical Engineering. In particular, I wanted to find a textbook that was well-used across a range of university engineering programmes, a textbook that most Engineering students would encounter during their studies. To that end, I read on-line course descriptions of first-year Engineering courses offered by major North American universities, looking specifically for titles of course textbooks; I searched for textbook titles on university bookstore websites; and I read blogs and message board posts about Engineering textbooks. I narrowed my search to Engineering Mechanics: Statics and Dynamics, by R. C. Hibbeler, and published by Pearson Prentice Hall.

Oxford. The Oxford textbook, Oxford English for Electrical and Mechanical Engineering, is from one of the leading publishers in English language education textbooks. It is a 190 page text comprising 30 units organized around various engineering topics. The back cover of the textbook explains that it has been designed specifically for tertiary level studies and aims to develop students' reading, writing, speaking, and listening skills. The chapter readings are described as authentic, meaning that they were written for real-world communication purposes as 
opposed to being created specifically as language pedagogy devices. This text is careful not to make outright predictions of student behaviour based on the course and its aims, and, instead, infers that it prepares students for discipline-specific English. The Oxford website page that describes this book observes that the book has been "[d]esigned for easy use by the non-specialist teacher" ("Oxford English for Electrical and Mechanical Engineering", 2013).

Garnet. The second EAP textbook is entitled English for Mechanical Engineering in Higher Education Studies and is published by the British publishing house Garnet. It is 130 pages long and includes 12 units, each organized around a topic in engineering. In its introduction, the text writers state that the aim of the book "is to teach students to cope with input texts, i.e., listening and reading, in the discipline" (p. 3). This textbook is part of a series that won the Duke of Edinburgh English-Speaking Union English Language Award for 2009.

Hibbeler. Engineering Mechanics: Statics and Dynamics, written by R. C. Hibbeler, is 718 pages long and divided into 22 chapters. The first 11 chapters are concerned with 'statics' while the remaining chapters focus on 'dynamics'. This is a canonical undergraduate engineering textbook, now in its 13th edition (although I analyzed the little-changed 200711 th edition due to accessibility issues), and a core course component at a number of universities, including Carleton. In fact, here at Carleton, the engineering department offers a first-year course built around this particular textbook. The course is required, and all engineering students, regardless of specialization, must pass it in order to proceed in the discipline.

\section{Data selection}

I considered carefully the data I should analyze. I decided to focus my attention on expository language, or the explanatory language that textbooks use, and omit analysis of instruc- 
tional language, the sets of instructions that precede learning tasks, in order to focus on each textbook's representation of Engineering subject matter since my overall purpose was to investigate claims that the EAP textbooks prepared students for discipline-specific language. In practice, this meant eliminating much more language from the EAP texts since both rely heavily on instructions. Using the expository text and chapter topics in the EAP textbooks as a starting point, I searched through the Statics and Dynamics textbook for matching content. I selected that content from Statics and Dynamics which most closely corresponded to the EAP content and was able to find appropriate matches for all of the EAP material.

In selecting the visual data, I decided to include all the visuals in each EAP chapter since the overall number of visual elements was low. 1 chose the visuals from Statics and Dynamics that were associated with the topic at hand. As much as possible, I also tried to select data of consistent length, with similar numbers of sentences, paragraphs, and visual elements so that, during analysis, I could discern patterns of similarity or difference more accurately. Below, I describe the data specific to each text.

Oxford. I selected the five-page chapter "Forces in Engineering" for analysis because the topic and content of this chapter matched topics and content from Statics and Dynamics. This chapter contains five expository paragraphs: four of the paragraphs comprise the whole of one reading and one paragraph stands alone. I selected for the verbal analysis the four connected paragraphs in order to keep the data size manageable. This chapter also includes eight visual elements, all of which were analyzed.

Garnet. All data were selected from the eight-page "Friction" chapter because topic and subject matter from this chapter matched topics and content in Statics and Dynamics. The chap- 
ter contains two expository readings; one made up of three short paragraphs and the other seven paragraphs long. I selected four paragraphs for analysis, two from the three-paragraph piece, and two paragraphs from the longer reading in order to maintain a reasonably consistent amount of verbal data among textbooks. The chapter includes five visual elements, all of which were analyzed.

Hibbeler. Two sets of data were compiled from this text, the first set to match the Oxford "Forces in Engineering" chapter and the second set to correspond to Garnet's "Friction". The first set, which for the sake of simplicity I will refer to as Hibbeler: Forces, I selected to correspond to the Oxford reading "Forces in Engineering". The Oxford reading discusses buoyancy, elasticity and springs, mass and weight, and friction, and so, in selecting text from Hibbeler, I was looking for those topics specifically. I found corresponding content in two different chapters. Two stand-alone paragraphs are from the 15-page introductory chapter and their titles explain their content: "Mass" and "Force". I selected a third stand-alone paragraph, entitled "Springs", from the 33-page chapter three. Six visuals from chapter three, one associated directly with the verbal text and five spatially close to the verbal text and associated with the 'forces' topic, provide the visual data. Although the most important criteria in choosing data for analysis was to maintain consistent subject content across the data-sets created from each textbook, I also tried to create similarly sized data-sets. The purpose in creating data-sets with similar numbers of clauses and visuals was to avoid analytical results skewed by size differences in data-sets.

The second set of data, which I will refer to as Hibbeler: Friction for reasons of consistency, I selected to correspond with the Garnet readings. The first Garnet reading is concerned with the co-efficient of friction and the second reading is about friction more generally; there- 
fore, in Hibbeler, I was looking for a general discussion of friction and content about the coefficient of friction. I found both in chapter eight, entitled "Friction", and from its 61 pages selected two paragraphs and part of a third from the opening pages of the chapter. The first paragraph I selected is part of a larger two-paragraph segment that discusses friction in a general way, while the second paragraph is a stand-alone paragraph that continues the general discussion of friction. The third paragraph I selected is about the co-efficient of friction, and I chose half of that paragraph for analysis in order to correspond as closely as possible to the Garnet co-efficient content. Six visuals are directly associated with the verbal text, and all were selected for analysis.

\section{Data analysis}

I selected a multimodal approach to data analysis in order to have a consistent analytical perspective towards the verbal and visual meaning-making modes I encountered in my data. The multimodal approach enables examination of any meaning-making mode, or semiotic system, along similar semantic dimensions, and thus recognizes that visuals and other non-linguistic semiotic systems make meanings as important as linguistic meanings in many academic genres (Hyland, 2009). With this approach, I incorporated inter-related frameworks to analyze the verbal and visual modes: the SFL framework for linguistic analysis of the verbal mode and a visual framework informed by Kress and van Leeuwen's theories of visual design. The multimodal approach also allows for investigation into the ways that modes work together, in multimodal ensembles, to create meaning, meanings that are often greater than the sum of their parts (Lemke, 2002, p. 303). This type of analysis I refer to as an intersemiotic analysis. While theories that explain the construction of multimodal ensembles are still being worked out within the discipline, I 
decided to draw from recent theorizing by Unsworth and Cléirigh (2009) because their theory is the most recent to be put forward and thus addresses the deficiencies of earlier theories.

Regarding the mathematical mode, I did not analyze it as a separate meaning-making mode for two reasons. First, the EAP textbooks contain so little mathematics that such an examination would have been futile. Furthermore, as Shaw (2006) contends, the role of the discourse analyst is not to investigate the mathematics itself but to understand the role it plays in the larger textual environment. Therefore, in recognizing that across all four data-sets the mathematics is consistently integrated into either the verbal text or the visual images, I decided, following Shaw, to include the mathematics as part of the linguistic and visual analyses. For example, when a mathematical item or expression was integrated into verbal text, I analyzed it as a linguistic item, and, conversely, when it appeared as part of a visual element, I analyzed it as a visual item. In reporting the analyses, however, I distinguish the mathematical items by analytical unit and present the mathematical findings separately. This approach to the mathematical analysis thus re'veals the roles mathematics plays in how each text represents subject content. I also felt that this flexibility in analysis - the ability to isolate mathematical items as part of the linguistic and visual contexts within which they express their meaning - was an advantage of selecting theoretically related multimodal approaches to analysis.

Since my goal was to discover how each text represents its subject content, one of my first decisions was to define subject content and decide how to investigate it. Subject content, in lay terms, is simply 'what something is about' or, in a phrase common to SFL, "who is doing what to whom, when, where, why, and how" (Eggins, 2004, p. 110). In SFL, the meanings that represent these experiences in language are realized through processes (or verbs), participants (or 
people, place, things), and circumstances (prepositions and adverbs that express time, quality, manner, and so on). Therefore, I selected those analyses that would best reveal verbal processes, participants, and circumstances. In all, I selected the three verbal analyses of transitivity, reference, and lexical relations. For the visual analysis, experiential meanings can be captured in a representational analysis, which I accordingly selected, along with a coding orientation analysis to gain a more general indication of how each text interprets experience.

I started the analysis by examining the verbal mode first, completing each analysis textby-text (rather than doing all three linguistic analyses for each text) before moving on to the next analysis. The reason for approaching the analysis this way was consistent with my overall goals to track similarities and differences in representations across the texts. Completing all the analyses for one text before moving on to complete all the analyses for the next text would have obscured emerging similarities or differences. I completed the visual analysis in a similar manner before the final multimodal analysis. Finally, I compiled findings into tables in order to highlight similarities and differences among the texts and to reveal larger patterns.

\section{Analytical Framework}

In this section, I present the analytical framework that I employed to analyze my data and develop my findings. This framework is presented in three parts. The first part describes each of the three verbal analyses I used: the transitivity analysis, the reference anlaysis, and the analysis of lexical relations. The second part describes the two visual analyses that I used: the representation analysis and the coding orientation analysis. Finally, I present the intersemiotic system of intermodal identification that I employed in order to understand the construction of verbal-visual ensembles. 


\section{Verbal analyses}

Transitivity. The three functional meanings that SLF sees language as expressing are the ideational, the interpersonal, and the textual. The ideational metafunction includes two components: experiential meanings, meanings that represent reality, and logical meanings, those which are concerned with how meanings are developed between clauses. While a logical analysis would have been informative, it is beyond the scope of this study. Since my interest is in determining how the EAP textbooks and the Engineering textbook represent their subject matter, I chose to analyze experiential meanings, the meanings that represent reality and show who is doing what to whom. This analysis allows me to capture the people, places, things and the actions and events that comprise the texts' subject matter. Experiential meanings are expressed through the system of transitivity, a system which describes the verbal processes used to represent reality. Following Halliday (1985/2004), processes are broadly classed into three groups, each group further divided into two types, as follows. First, processes of doing describe concrete actions and events and are generally classified as material processes. However, if such a process describes a physiological action of a conscious being, it is a behavioural process. The second classification involves processes of sensing, divided into mental processes that encode meanings of thinking, feeling, or perceiving and verbal processes that encode meanings of saying or verbalizing. The third classification, processes of being, encode the meaning that something is. The two groups here are existential processes, encodings that simply observe that something is, and relational processes, processes that relate one thing to another. Each process type is also semantically associated with particular participants, or people, places, and things, and may be associated with particular circumstances. Processes are revealed through an analysis of verbal groups, the partici- 
pants that each process thus represents are realized through nominal groups, while the circumstances each brings to the topic are typically realized as adverbial groups or prepositional phrases (Eggins, 2004). Each component of the transitivity analysis will develop the texts' representation of the reality they are encoding. Processes will reveal tendencies to the construal of experience as doing, sensing, or being in the world. Each process's participants will be constructed accordingly: material processes construct Actors and Goals; mental processes construct Sensers and Phenomenon; relational attributive processes construct Carriers and Attributes, while relational identifying processes construct Token and Value participants. As well, circumstantial information regarding extent, spatial and temporal location, manner, and cause will reveal topic details and further specify areas of concern.

Reference analysis. The next two verbal analyses I carried out, a reference analysis and an analysis of lexical relations, are normally analyses of cohesion, and thus conceptualized as textual analyses. These analyses describe how textual cohesion develops among groups of semantically related words across lengths of text. However, for this study, I focus on the semantic relations derived from the use of these cohesive resources and what these relationships reveal about the experiential content of the text. The reference analysis investigates the grammatical resource of reference which refers to how a writer introduces participants and follows them through the text. For example, a force might be introduced and then might show up again as it, it, this force, and so on. In this way, a reference analysis, called a 'reference chain', tracks participants, determines major participants according to chain length, and thus reveals which participants are of most concern to the topic at hand. Reference chains also show how participants may be modified through the course of the text, for example a force, it, it, this applied force. As well, 
this analysis shows how the text refers to its participants, in two different ways. First, references to participants are identified as one of four grammatical types: references may be anaphoric or backward-looking (the participant is named and, later in the text, referred to such that the reader needs to look back in the text to retrieve the first mention), forward-looking or cataphoric, esphoric or immediately following the initial mention, or they may be whole text references that refer to a sequence of events rather than to a single participant. Second, participants are also identified by their grammatical reference item, the pronouns and articles used to refer to the participant, such as $a$ in $a$ force. The most common reference items are definite articles and pronouns. The reference analysis, then, will allow me to identify major participants and state how participants are grammatically tracked through the text.

Lexical relations analysis. The lexical relations analysis investigates lexical resources as opposed to the grammatical resources of the reference analysis. Lexical relations refers to the related groups of content words (nouns, verbs, adjective, adverbs) that build semantic ties across the text and connect the text to its field or ideational domain. This analysis describes how content words group together in one of two ways to build lexical strings. First, taxonomic strings are built around either classification groupings or composition groupings. Classification groupings include class/sub-class relationships, such as book/textbook, and relationships of contrast, synonymy, and repetition. Compostion groupings are part/whole relationships, such as book/page. Second, expectancy strings, or collocations, refer to the tendency of two or more words to associate or share a similar place in the text. For example, if we come across the word mass in an instructional text, we would not be surprised to see weight show up a few words later. Technical words often fall into collocation strings (Halliday, 1985/2004). A text's lexical strings reveal how 
the writer represents an area of experience and how that ideational context is developed (Eggins, 2004). Major strings thus identify areas of central focus while the length of a string indicates the degree of focus on the topic, with longer strings normally evidencing stronger focus. The length of an item in a string, whether one word, or two or more, also points to patterns in topic development. Texts can also be understood in terms of the type of resources each selects to build its semantic ties. For example, scientific and technical texts often represent reality in taxonomies with detailed classifications (Eggins, 2004; Halliday \& Martin, 1993; Martin \& Veel, 1998).

\section{Visual analyses}

Representation analysis. The visual mode expresses the three functional meanings, similar to the three metafunctional meanings expressed verbally, but adapted to the visual context. Representational meanings are similar to the experiential meanings of language and visually encode real world actions and objects. Interactional meanings correspond to language's interpersonal meanings and visually encode the relationships between image producers and image viewers. Compositional meanings are similar to the textual meanings of language and visually connect representational and interactional meanings to one another (Kress \& van Leeuwen, 2006). However, since my larger concern is to determine how subject content is represented visually, my focus will be on analyzing representational meanings in order to reveal how the visuals in the analyzed texts represent their subject matter.

Representational meanings are broadly classed as narrative processes or as conceptual processes; narrative processes encode the concrete actions and events of the experienced world while conceptual processes are concerned with visual representations of concepts and ideas. The primary visual distinction between these two major process types is the presence or absence of 
vector lines. Vectors are linear representations that signify action, processes, events, or some other dynamic meaning. Vectors may be readily discernible, such as arrows on a diagram, or may be inferred, such as a line that might be drawn from a person's gaze towards the object of the gaze. Once the presence of a vector has been established, the nature of the action can be further discerned by noting the number of participants in the action and their relationship to the action and to one another. Thus, narrative action is either non-transactional and involves just one participant, an Actor, or is transactional and includes at least two participants, an Actor and a Goal. With conceptual processes vectors are absent and representations are concerned with generalized forms and meanings, broadly grouped as classification or analytical processes. Classification processes are realized as simple or complex taxonomies while analytical processes are realized as part/whole relationships. Analytical processes are further defined as exhaustive or inclusive, representing an entire participant or just a portion, and as topographical or topological, representing spatial arrangements accurately or showing how participants are logically connected. Participants in conceptual analytical processes are Carriers and Attributes. Visual processes thus reveal tendencies to the construal of experience as doing or being in the world and also clarify the dimensions of that experience. Participant roles identify the texts' objects of interest.

Coding orientation analysis. The second visual analysis I conducted, to determine coding orientations, is an extension of the interactional metafunction which encodes relationships between image producer and viewer. This analysis is normally used as an indicator of credibility in how visual elements encode reality (and thus draws relationships of believability between producer and viewer) but here I use it to gain insight into how each text represents itself. The underlying concept in this analysis rests on the observation that some messages are seen as more 
credible or more reliable than others. Without going into a discussion of truth values in messages, a fascinating area but beyond the scope of this study, certain configurations of visual features are utilized as modality markers, markers of credibility. These modality markers align viewers with one of four approaches or orientations to credibility: a naturalistic approach, a technical or scientific approach, a sensory approach, and an abstract approach. (Sensory and abstract approaches are not discussed here since neither orientation was found in the data.) For example, a visual that conveys reality naturally, such as a realistic photograph of a device or tool, finds its credibility in the naturalistic presentation of the represented object: the colours are true-to-life, as are the context and the detailing. The photograph, of course, may be digitally modified or adjusted, but as long as it maintains the modality markers of naturalistic representation, it encodes a naturalistic orientation and thus the credibility associated with the natural world. Should the producer of the visual alter the modality markers enough, for example omit colour, context, and detail, the overall orientation changes. In this example, the reduction of colour, context, and detail changes the orientation from naturalistic to technical or scientific since the technical orientation gains its credibility from the authority ascribed to models, templates, and blueprints. The final credibility of the photograph may not have changed, in that a viewer still believes the photograph to be representing a truthful likeness, but the credibility now comes from the world of science and technology, not from the natural world. I selected this analysis in order to uncover the different coding orientations that each text chooses in its efforts to create credibility. The chosen orientation will reveal the experiential world - naturalistic or technical - that each text selects to encode credibility, thus highlighting that category of experience as significant enough to convey credibility. 


\section{Intersemiotic analytical system}

The framework of analysis that I employed here is drawn from recent theorizing put forward by Unsworth and Cléirigh (2009). I briefly outlined their system of intermodal identification in the literature review above, but here I will provide a fuller description. This theory offers an explanation of how the verbal and visual modes interact to jointly construct experiential meaning in multimodal ensembles. This interaction is modelled on the grammatical concept of relational identification as described by systemic functional linguistics. This concept is most commonly seen in sentences with 'to be' or 'to have' verbs, sentences that identify one entity with some other entity, in the sense of She is the leader, for example, where she is identified with the leader. In recognition of this meaning distinction, SFL terms the participants in clauses like this one as 'Token', the holder of a meaning or value, and 'Value', the content or expression of that value. In this example, she is the Token and the leader is the Value. Unsworth and Cleirigh extend this identifying concept beyond language in order to describe interactions among words and images. Thus, in multimodal ensembles, visual images function as the Token while the written language functions as the Value, with each mode identifying the other. For the viewer, either mode may, in fact, identify the other, depending on which representation is most familiar (although producers will normally assume that one is more familiar than the other). When the visual is the familiar element, it is said to visualize the language; when the language is the familiar element, it glosses the visual. Images can identify language in two ways: an image may identify a language element or an image may identify a language configuration. A language element is normally a nominal participant such as a person, place or thing, while a language configuration is normally a clausal-like semantic arrangement including a process (verbs), participants (nouns), 
and circumstances (adjectives and adverbs). Images can identify language elements in three ways: first, in intensive identifications, the image visualizes non-verbalized qualities of shape, colour, texture, and so on. In Figure 2, for example, the image, which is identified by the caption A block of uniform weight, visualizes the colours of the block as grey and white and the shape of the block as rectangular. In the second type of identification, possessive identification, the image visualizes additional, non-verbalized parts of the verbally identified participants. In Figure 2 , the image visualizes the block's two parts. In the third type of visual identification, circumstantial, the image visualizes the non-verbalized location of the participant's parts. Again referring to Figure 2, the diagram visualizes the location of the block's two parts as an upper half and a lower half. Each of these three types of intermodal identification is consistent with notions of visual affordances (Kress \& van Leeuwen, 1996 \& 2006) and epistemological commitments (Kress \& Bezemer, 2008, Kress, 2010), described earlier.

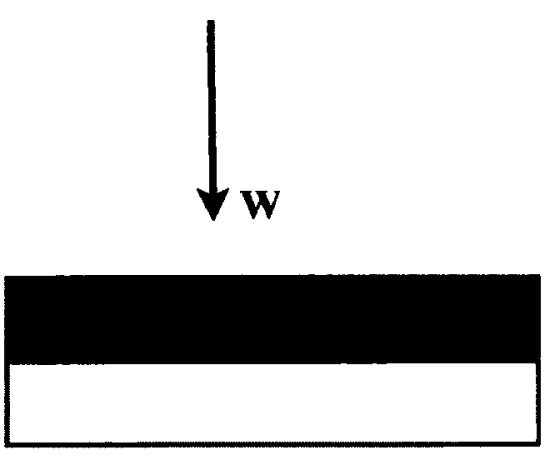

Figure 2: A block of uniform weight

Images also identify or visualize language configurations in three ways: first, in manner identifications, the way that participants are located in the visual can show a non-verbalized aspect of manner, or how one participant is related to another. For example, in Figure 2, if the caption were changed to read Two blocks of uniform weight, the image would be visualizing the manner in which the two blocks are related: the grey block as resting on top of the lighter one. 
The second type of configuration, causal: result or consequence, shows how the visual represents the result or consequence of the language configuration. For example, if the caption in Figure 2 were changed to read Glue the white box to the darker box, the visual would be showing the result of that language, namely that the grey box has been glued on top of the white box. Thirdly, in causal: reason or condition identifications, the way that visual participants are located can show non-verbalized reasons or conditions of the language configuration. To refer again to Figure 2, the visual participants, the block and the downward arrow, show a reason for the language configuration a block of uniform weight. The downward arrow and the $W$ in the diagram indicate that the reason for this uniform weight is the downward force of gravity. As Unsworth and Cléirigh observe, meanings developed in this type of interplay between two modes are consistent with Lemke's ideas of multiplying meaning (Lemke, 1998). That is, intermodal identification of language configurations may realize meanings that are not constructed by either the visual or verbal mode alone. In the causal: result example above, the diagram alone does not indicate a representation of two boxes glued together, while the language alone does not indicate a representation of the white box on top of the dark box. Applying the Unsworth and Cléirigh (2009) model to my analysis of multimodal ensembles enables an identification of the type of intermodal identification that each of the texts constructs. Aside from being able to compare how texts make their intersemiotic meanings, the meanings thus derived are important from a language-learning perspective. As Unsworth and Cleirigh (2009) observe, the more unfamiliar and technical the topic, the more important the intermodal identification. 


\section{Chapter Four \\ Analysis}

This chapter is organized into three main sections, each addressing one research question. The first section reports the findings of the three verbal analyses, and thus establishes the main verbal subject matter of each text. The second section reports the findings of the two visual analyses, thus establishing visual subject matter, while section three discusses how each text constructs meaning in multimodal ensembles. Each analysis closes with a brief summary to synthesize findings. Samples of the analyses can be found in the Appendix, along with the verbal data from each textbook.

\section{Verbal Representations of Subject Matter}

\section{Transitivity analysis}

In a general sense, experiential meaning refers to how words in a sentence or clause represent reality or, in a favourite SFL phrase 'who is doing what to whom'. Experiential meanings are expressed through transitivity, the process or verbal system, and through circumstances. The focal point of the transitivity analysis is the process, or verb, and the various participants that are associated with it. English grammar categorizes processes functionally and structurally such that each process type also delineates participant roles. In this way, a transitivity analysis provides detailed information about the expression of reality as 'doing', 'sensing', or 'being' content in the text and about the participant associated with each process type. Circumstantial elements provide additional experiential content and may be added to any of the process types. The use of nominalization, the process by which verbs are turned into nouns, in the expression of participants and circumstances is also analyzed since this usage adds experiential content to a text. 
Oxford. Of the 27 processes realized in this text (analysis presented in Appendix $\mathrm{E}$ and summarized in Table 1), almost equally half are material and half are relational. Material processes are about 'doing' and express ideas of one entity doing something, with the 'doing' often extending to another entity. Participant roles associated with material processes, then, are Actor, the doing entity, and Goal, the 'extended to' entity. Relational processes are concerned with 'being' and, here, are almost equally split between attributive relationals and identifying relationals. Attributive processes assign an attribute; thus, participants are Carrier, the entity that carries the attribute, and Attribute, the attribute itself. Identifying processes assign an identity; participants involved are Token, the holder of the identity, and Value, the assigned identity.

Table 1

Oxford process wordings

\begin{tabular}{|c|c|c|}
\hline \multicolumn{2}{|c|}{ Process } & Wording \\
\hline \multicolumn{2}{|l|}{ Material } & act $\times 2$, pulls $\times 2$, measured $\times 2$, exert, stretched, calibrated, give, oppose \\
\hline \multicolumn{2}{|l|}{ Mental } & solve, get, look \\
\hline \multirow[t]{2}{*}{ Relational } & Attributive & is $\times 3$, be, provides, has \\
\hline & Identifying & is $x 5$, equals, being \\
\hline
\end{tabular}

Circumstances are optional clausal elements and provide additional information. This text shows 12 circumstances, of which seven express spatial location and four express manner. Location circumstances are primarily concerned with definite location such as on the ship and in an object or with spatial motion such as upwards and downwards. All three manner circumstances are to do with measurement as means: in kilograms, in newtons, and in newtons, the unit of force. The text shows limited use of nominalization with only two nominalized words, weight and equilibrium. Both, however, are repeated: weight six times and equilibrium twice. 
Garnet. This text shows 33 processes (see Table 2) with relational and material processes dominating at 14 and ten occurrences respectively. Mental processes are also somewhat important here with six occurrences. The text, then, construes reality primarily in terms of being with a secondary focus on doings. Mental processes add a smaller thinking dimension. The relational processes of being are almost equally divided between attributive processes and identifying processes thus specifying these participants as Carriers of an Attribute and Tokens or holders of a Value.

Table 2

Garnet process wordings

\begin{tabular}{|c|c|c|}
\hline \multicolumn{2}{|c|}{ Process } & Wording \\
\hline \multicolumn{2}{|c|}{ Material } & $\begin{array}{l}\text { moved, created, resists, occurs, opposes, move, initiate, overcome, maintain, } \\
\text { reduced }\end{array}$ \\
\hline \multicolumn{2}{|c|}{ Mental } & considered, required, known, bear in mind, needed, required \\
\hline \multicolumn{2}{|c|}{ Verbal } & called \\
\hline \multicolumn{2}{|c|}{ Existential } & are, is \\
\hline \multirow{2}{*}{$\begin{array}{l}\text { Rela- } \\
\text { tional }\end{array}$} & Attributive & is $\times 3$, are $\times 2$, be, (ellipsis of 2 verbal groups) \\
\hline & Identifying & is $x 3$, depends, are, given \\
\hline
\end{tabular}

Circumstances are realized 13 times, with eight expressions of spatial location, two of temporal extent, and two of manner. Absolute and relative spatial locations are emphasized, with five realizations, and include in all bodies, in all machines and relative to one another. Circumstances of spatial motion are expressed twice, from a standstill and past the other, as are circumstances of temporal extent, with times and when. The text shows some use of nominalization, with five examples: functioning, speed, lubrication, design, and the thrice-repeated movement. 
Hibbeler: Forces. Of the 28 processes in this text (analysis presented in Appendix $\mathrm{E}$ and summarized in Table 3), the most common is the material process with 12 occurrences, followed by eight relational and five mental processes. The relational processes are mostly attributive with six participant Carriers holding Attributes. This text, then, constructs reality primarily as a matter of action or doings, with some emphasis on attributes of being and a smaller focus on thinking.

Table 3

Hibbeler: Forces process wordings

\begin{tabular}{|c|c|c|}
\hline \multicolumn{2}{|c|}{ Process } & Wording \\
\hline \multicolumn{2}{|c|}{ Material } & $\begin{array}{l}\text { provides, exerted, occur, pushing, occur, separated, pulls, push, stretch, use, } \\
\text { change, deformed, measured }\end{array}$ \\
\hline \multicolumn{2}{|c|}{ Mental } & compare, considered, needed, determined \\
\hline \multicolumn{2}{|c|}{ Existential } & is \\
\hline \multicolumn{2}{|c|}{ Causative } & manifests \\
\hline \multirow{2}{*}{$\begin{array}{l}\text { Rela- } \\
\text { tional }\end{array}$} & Attributive & is, is, is, has, is, has, characterized \\
\hline & Identifying & include, is \\
\hline
\end{tabular}

The text shows 14 circumstantial elements in an almost equal expression of spatial location and manner. Location circumstances are almost all concerned with expressing definite location as on a wall, on another, and between the bodies. Manner circumstances here express means and quality. Circumstantial means typically describe how a process takes place and, in this text, include these complex examples: in direct proportion to the force acting on it and from the difference in the spring 's deformed length l and its undeformed length lo. Circumstantial quality descriptions further characterize processes with this text providing one simple and one complex example: physically and by its magnitude, direction, and point of application. There are 11 ex- 
amples of nominalization in this text, which occur in three transitivity processes and in circumstance roles.

Hibbeler: Friction. With 21 of its 37 processes material, this text shows a focus on actions or doings. A further eight mental processes and four relational attributive processes construct experience in terms of thinking and attributes of being (see Table 4).

Table 4

Hibbeler: Friction Process Wordings

\begin{tabular}{|c|c|c|}
\hline \multicolumn{2}{|c|}{ Process } & Wording \\
\hline \multicolumn{2}{|c|}{ Material } & $\begin{array}{l}\text { act } \times 5 \text {, develop } \times 2 \text {, prevents or retards, directed, oppose, caused, resting, ex- } \\
\text { erts, balance, prevent, moving, exist, contributes, sliding, combine, create }\end{array}$ \\
\hline \multicolumn{2}{|c|}{ Mental } & defined, explained, shown, considering, considered, reveals, determined, seen \\
\hline \multicolumn{2}{|l|}{ Verbal } & called $\times 2$, expressed \\
\hline \multirow{2}{*}{$\begin{array}{l}\text { Rela- } \\
\text { tional }\end{array}$} & Attributive & is $x 2$, develop, necessary, be \\
\hline & Identifying & - \\
\hline
\end{tabular}

Circumstances are realized 20 times with circumstances of spatial location and manner dominating. Spatial circumstances express definite locations seven times with examples such as at points of contact with other bodies, on the free-body diagram, and at each of the protuberances. Circumstances of spatial motion include to the left, to the right, and upward while relative and absolute circumstances include relative to a second body or surface and at all points of contact. Five manner circumstances are concerned with means, or how something is done, and include by pulling horizontally, close examination, and mathematically. Circumstances here may also be roughly categorized according to head noun. In this way, nine circumstances locate a surface or point of contact in space and time, revealing an attention to edges and points not other- 
wise seen. This text also relies on nominalization with nine examples occurring almost entirely as material participants and circumstances.

Summary. Table 5 below summarizes the transitivity analysis by presenting the number of clauses of each process type for each text. As the table shows, the two EAP texts and the two Hibbeler texts demonstrate different patterns in their representations of reality. The Hibbeler texts represent reality mostly as a matter of 'doing', while the Oxford and Garnet texts construct reality in terms of 'being' and 'doing'. In the Hibbeler texts, the high proportion of material processes reveals that the material world of actions, events, and participants is of central focus. The Oxford and Garnet texts use material and relational processes almost equally, showing a split concern for the material world and the world of being. Of the relational processes, proportions of attributes and identifying types also differ across the four texts: Oxford and Garnet show almost equal proportions of attributive and identifying processes while, in the Hibbeler texts, attributive processes clearly dominate with no or almost no identifying processes. This difference in distribution of relational processes reveals a further difference in focus: Oxford and Garnet are interested in identifying and describing participants, while the Hibbeler texts are almost completely concerned with description. This difference marks one feature that characterizes technical language; technical language favours the attributive process and its descriptive capacity while everyday language tends to favour the identifying relational (Eggins, p. 108). 
Table 5

Process summary by number of clauses per text

\begin{tabular}{|c|c|c|c|c|c|}
\hline \multicolumn{2}{|c|}{ Process } & Oxford & Garnet & Hibbeler: "Forces" & $\begin{array}{l}\text { Hibbeler: "Fric- } \\
\text { tion" }\end{array}$ \\
\hline \multicolumn{2}{|l|}{ Material } & 11 & 10 & 13 & 21 \\
\hline \multicolumn{2}{|l|}{ Mental } & 3 & 6 & 4 & 8 \\
\hline \multicolumn{2}{|l|}{ Verbal } & 0 & 1 & 0 & 3 \\
\hline \multirow[t]{2}{*}{ Relational } & Attributive & 6 & 8 & 7 & 5 \\
\hline & Identifying & 7 & 6 & 2 & 0 \\
\hline \multicolumn{2}{|l|}{ Existential } & 0 & 2 & 1 & 0 \\
\hline \multicolumn{2}{|l|}{ Causative } & 0 & 0 & 1 & 0 \\
\hline \multicolumn{2}{|l|}{ TOTAL } & 27 & 33 & 28 & 37 \\
\hline
\end{tabular}

Another feature of technical and scientific language is nominalization (Eggins, 2004; Halliday, 1985/2004; Halliday \& Martin, 1993), the linguistic process that converts verbs into nouns. Once words are nouns, they can be specified and classified in ways that verbs cannot (for example, resistance as a noun, rather than as the verb resist, allows for different types of resistance to be specified - normal resistance, frictional resistance, and so on - and then classified). Table 6 names all occurrences of nominalized words in each text and reveals differences in how the EAP texts and the Hibbeler texts use nominalization. As the table shows, the Hibbeler texts contain significantly more instances of nominalization than the EAP texts, especially once the repeated items in Oxford and Garnet are accounted for. The presence of nominalized elements in Hibbeler demonstrates a focus on constructing participants that can be specified. For example, $a$ distribution in Hibbeler: Friction permits specifications such as a distribution of...normal force 
and $a$ distribution ... along the contacting surface. The use of nominalization helps characterize the language in the Hibbeler texts as technical language.

Table 6 Nominalization summary, by number of occurrences and wordings

\begin{tabular}{|c|c|l|}
\hline Text & Number & \multicolumn{1}{|c|}{ Wordings } \\
\hline Oxford & 8 & weight x 6, equilibrium x 2 \\
\hline Garnet & 7 & movement x 3, speed, functioning, lubrication, design \\
\hline $\begin{array}{c}\text { Hibbeler: } \\
\text { "Forces" }\end{array}$ & 11 & $\begin{array}{l}\text { measure, resistance, interaction, action, a "push", a "pull", contact, attraction, } \\
\text { application, position, difference }\end{array}$ \\
\hline $\begin{array}{c}\text { Hibbeler: } \\
\text { "Fric- } \\
\text { tion" }\end{array}$ & 9 & $\begin{array}{l}\text { resistance, slipping, motion, distribution, irregularities, resultant, understand- } \\
\text { ing, equilibrium, examination }\end{array}$ \\
\hline
\end{tabular}

All four texts show spatial location circumstances as dominant, as shown in Table 7's summary of circumstances. However, the Hibbeler texts show a greater number of circumstances in proportion to their length, signifying a greater concern for locating processes in space and determining how processes occur. In both Hibbeler texts, the dominant location circumstances are those of definite location, indicating a concern with specific positions that locate processes and their participants in space. The prevalence of manner circumstances indicates specificity in the way processes are executed.

Overall, the transitivity analysis reveals that the Hibbeler texts are concerned with the action, events, and participants of the material world and with specifying that world through nominalization and circumstantial detail. The Oxford and Garnet texts are concerned with both the material world and the world of being, and attend to the broader identification of participants in language that is more consistent with the everyday (Eggins, 2004). 
Table 7

Circumstances summary

\begin{tabular}{|l|l|l|l|l|l|}
\hline \multicolumn{2}{|c|}{ Circumstance } & \multicolumn{1}{c|}{ Oxford } & \multicolumn{1}{c|}{ Garnet } & Hibbeler: Forces & Hibbeler: Friction \\
\hline \multirow{3}{*}{$\begin{array}{l}\text { Location: } \\
\text { Spatial }\end{array}$} & definite/indefinite & $3 / 0$ & $0 / 1$ & $5 / 0$ & $7 / 0$ \\
\cline { 2 - 7 } & absolute/relative & - & $3 / 2$ & - & $1 / 2$ \\
\cline { 2 - 7 } & rest/motion & $0 / 4$ & $0 / 2$ & $1 / 0$ & $0 / 3$ \\
\hline \multirow{3}{*}{$\begin{array}{l}\text { Location: } \\
\text { Extent }\end{array}$} & spatial & - & - & 1 & 1 \\
\cline { 2 - 7 } & temporal & - & 2 & - & - \\
\hline \multirow{3}{*}{ Manner } & means & 3 & 1 & 2 & 5 \\
\cline { 2 - 7 } & quality & 1 & - & 1 & - \\
\cline { 2 - 7 } & comparative & - & 1 & 1 & 1 \\
\cline { 2 - 7 } & role & - & - & 1 & 1 \\
\hline Cause & 1 & 1 & 1 & 20 \\
\hline TOTAL & 12 & 13 & 13 & 37 \\
\hline Number of Ranking Clauses & 27 & 33 & 28 & \\
\hline
\end{tabular}

\section{Reference analysis}

A reference analysis tracks a text's participants and grammatically establishes how participants are referenced throughout the text. An initial participant, normally a nominal item, is identified and all ensuing references to that participant are tabulated into a reference chain. Resulting chains thus identify major participants by chain length. At the same time, types of reference are shown as anaphoric, cataphoric, esphoric, or whole text. In this analysis, since the Hibbeler samples are not all drawn from consecutively connected text, all reference chains are established by paragraph.

Oxford. The analysis is presented in Appendix F while Table 8, below, summarizes the main findings. Reference chains construct force as the major participant, and the following chain 
is a typical example: the gravity force, the buoyancy force, $B$, the resultant force. Two other chains include a mathematical reference to force, including this example, the longest chain in the text: another very important force, the one, force, force, this upward force, $F I$. While this chain includes an unusual four-item reference, another very important force, the more complex reference is the mathematical $F 1$. Other important participants include weight, springs, and friction with reference chains as follows (headword added for clarity):

Weight: a weight, the weight, that weight, the weight of the block, W.

Springs: a spring, springs, they, the spring, the spring.

Friction: friction, friction, it, the friction force, $F$.

By paragraph, reference chains of major participants tend to be five to six items long, with each item in the chain typically composed of one to three words. Mathematical items occur in three chains and are usually a single alphabetic symbol.

Turning to the number of references per clause, most clauses contain one reference item, several contain two references, and only a few contain three references. No clause establishes more than three reference ties. Reference ties tend to be anaphoric: a presuming reference refers back to the initial referent. The presuming reference words are most often the definite article 'the'; paragraph one uses only this type of reference tie while the remaining paragraphs also include pronouns and simple repetition.

Garnet. The major participant in the Garnet samples, again not surprisingly, is friction, as summarized in Table 8. Friction is referenced five times in paragraph four and four times in paragraph three:

friction, some friction, friction, the friction, friction. 
friction, friction, it, friction.

Other important participants, coefficient of friction and objects, construct the following chains. Note that the coefficient of friction chain is the most complex chain in the Garnet analysis as it includes an unusual 4-item reference and a mathematical symbol (headwords added for clarity):

Coefficient of friction: the coefficient of friction, $\mu$, it, a high coefficient, a low one.

Objects: two objects, one object, the other, the objects.

Major participant chains are typically four to five items long. Items in each chain are usually composed of one to three words or one mathematical symbol. Clauses typically contain one reference item, several contain two references, and only three clauses contain three items. No clause establishes more than three reference ties. Reference ties tend to be anaphoric: a presuming reference refers back to the initial referent. Presuming reference words include definite and indefinite articles and pronouns.

Hibbeler: Forces. The analysis, summarized in Table 8, below, and presented in Appendix F, shows that the long "Springs" paragraph develops at least three major participants: spring, length, and distance. Spring is the most complexly developed of all participants with this 12 -item chain specifying its name, position, and length:

a linearly elastic spring, the length of a spring, it, the 'elasticity' of a spring, a linearly elastic spring, its unloaded position, the spring's deformed length, its undeformed length, the spring, it, the spring, it.

Other major participants, length and force, build complex lexical and mathematical chains nine items and seven items long, respectively (headwords added for clarity): 
Length: the length, the spring's deformed length, 1 , its undeformed length, lo, $\mathrm{s}=1-10$, undeformed length, lo $=0.4 \mathrm{~m}, 1=0.6 \mathrm{~m}$.

Force: the force, the magnitude of force, $F=k s, F, F, a$ force, $F=k s=(500 \mathrm{~N} / \mathrm{m})$ $(0.6 \mathrm{~m}-0.4)=100 \mathrm{~N}$.

Chains tracking major participants vary in length from three to four items long to the 12item spring chain. Items in chains are typically three to four words long while mathematical items are typically complex in that they are composed of one or more symbols or terms. Clauses most usually contain one reference tie, but two clauses show two and three ties and three clauses have four ties. While anaphoric referencing is most common, all reference types, backwardlooking, forward-looking, and inferencing, are realized here. All presuming reference items are used, as well: definite and indefinite articles and pronouns.

Hibbeler: Friction. As summarized in Table 8, below, the analysis shows that force, body, and block are constructed as the main participants in these samples, with references to force occurring across all three paragraphs. The thee chains identify six sub-types of force, realize force in highly specified descriptions, such as the limiting static frictional force $F_{s}$, and usually add a mathematical dimension to force:

Paragraph 1: a force of resistance, this force.

Paragraph 2: normal force $\Delta \mathrm{Nn}$, frictional force $\Delta \mathrm{Fn}$, the normal forces, the frictional forces, the applied force $\mathrm{P}$, these frictional and normal forces, reactive forces $\Delta R n$, these forces, each reactive force, a frictional component $\Delta F n$, a normal component $\Delta \mathrm{Nn}$. 
Paragraph 3: the limiting static frictional force Fs, the resultant normal force $\mathrm{N}$, the normal force N, frictional force Fs, N.

The other major participants, body and block, are not as complexly expressed and are presented in lexical terms only in smaller six-item and five-item chains. Nevertheless, block is specified in three phrases describing its weight, section, and depiction (headwords added for clarity):

Block: a block of uniform weight, the upper portion of the block, the free-body diagram of the block, the block, block.

Body: a body, the body, a second body, it, other bodies, the body.

Aside from the one 11-item force chain, major participant chains tend to be composed of five or six items. Most participant items in a chain are three- to five-word phrases; typical mathematical items are made up of two to three symbols. Most clauses have one reference tie, nine clauses have two ties, and six clauses have between three and five ties. Reference ties are most often anaphoric but all reference types are realized as are all presuming reference items.

Summary. Findings of the reference analysis are summarized in Table 8, below. While the analysis shows that the texts share some participants and grammatical features in how reference chains are constructed, more significant are the differences that exist between the EAP texts and the Hibbeler data-sets. Concerning similarities, the four texts all demonstrate preferences for anaphoric references and all use the full range of pronouns and definite and indefinite articles as presuming reference items. As well, the two 'forces' data-sets, Oxford and Hibbeler: Forces, construct force and spring as major participants. Similarities end here though as the texts show differences in their construction of major participants. The other two major participants in Oxford 
are weight and friction, that along with force, demonstrate a concern with generic participants.

Hibbeler: Forces reveals a concern with specified and detailed participants such as a linearly elastic spring. The length of the Hibbeler: Forces reference chains also shows a clear focus of attention on specifics of the spring, most particularly its length. A similar pattern is seen in the 'friction' data-sets. Garnet's major participants are friction, coefficient of friction, and objects. While the coefficient construction shows attention to specifics, the other two participants, friction and objects, are generic, and neither are further specified or developed. Hibbeler: Friction, on the other hand, constructs force as a major participant, specifying it in six sub-types with verbal and mathematical references for each. The 11 items in the force chain demonstrate a clear focus on that topic, and show special attention to mathematical symbols or names of forces, both simple, such as $P$ for applied force, and complex, such as $\Delta R n$ for reactive forces. Block is also developed as a major participant and, although not as specified as force, is described along three different dimensions. Overall, both Hibbeler texts construct mathematical items as major participants with 11 mathematical participants in major chains in Hibbeler: Friction and nine in Hibbeler: Forces. In comparison, Oxford shows four mathematical participants in major chains, and Garnet shows three. In brief, the Hibbeler texts show highly specified participants, numerous mathematical symbols and expressions as participants, and an overall complexity in the expression of mathematical items. 
Table 8

Reference analysis summary

\begin{tabular}{|l|l|l|l|l|}
\hline \multicolumn{1}{|c|}{ Chain Feature } & \multicolumn{1}{c|}{ Oxford } & \multicolumn{1}{c|}{ Garnet } & Hibbeler: Forces & Hibbeler: Friction \\
\hline $\begin{array}{l}\text { Head items of longest } \\
\text { chains (no. of items in } \\
\text { chain) }\end{array}$ & $\begin{array}{l}\text { force (6) } \\
\text { weight (5) } \\
\text { spring (5) } \\
\text { friction (5) }\end{array}$ & $\begin{array}{l}\text { friction }(5,4) \\
\text { coefficient of } \\
\text { friction (5, 2) } \\
\text { objects }(4,4,2)\end{array}$ & $\begin{array}{l}\text { spring (12) } \\
\text { length (9) } \\
\text { force (3, 7) } \\
\text { distance (5) }\end{array}$ & $\begin{array}{l}\text { force }(2,11,5) \\
\text { body (6) } \\
\text { block (7) }\end{array}$ \\
\hline $\begin{array}{l}\text { Typical word count } \\
\text { per chain item }\end{array}$ & $1-3$ & $1-3$ & $3-4$ & $3-5$ \\
\hline $\begin{array}{l}\text { Typical math symbol } \\
\text { count per chain item }\end{array}$ & 1 & 1 & $\begin{array}{l}\text { single symbols \& } \\
\text { expressions }\end{array}$ & $2-3$ \\
\hline $\begin{array}{l}\text { Total math participants } \\
\text { in major chains }\end{array}$ & 4 & 3 & 9 & 11 \\
\hline
\end{tabular}

\section{Lexical relations analysis}

The lexical relations analysis describes how words in a text are connected to one another and how they cluster together to build lexical strings. Since this analysis examines semantic ties across content words (nouns, verbs, adjectives, and adverbs), the obtained strings reveal the text's experiential content or topic and permit identification of major focuses in content. The length of the string shows the degree of focus on that topic. This analysis also establishes the nature of the lexical relations as one of three types: as classification relationships of hyponymy, contrast, synonymy, or repetition, or as composition relationships of meronymy, or as collocation relationships.

Oxford. The lexical relations analysis is summarized in Table 9, below, and shows that lexical strings are most often built around the key textual participants force, weight, and springs, establishing these three focuses as central topics of the text. These topics are usually presented in classification relationships of synonymy and repetition or in collocation relationships marking technical language. The most common pattern of synonymy ties together a mathematical symbol 
and a lexical item as equivalent. For example, $B$ is synonymous with buoyancy force, $F 1$ with upward force, and $W$ with weight. Occasionally, one of the topic words is represented with a lexical synonym, as in weight: gravity force. Lexical strings are also commonly built around repetition. For example, force is repeated 14 times across three paragraphs and weight seven times across two paragraphs. Collocation relationships that bring together technical words or mathematical symbols are also employed to express the main topics. Hence, spring balance, calibrated, and newtons can be seen as technical collocations of spring while buoyancy, elasticity, gravity, and friction form a quasi-technical grouping of forces. The collocation measured, mass, kilograms is another example of quasi-technical language. The mathematical collocation includes $W, B, F 1, R$, and $F$.

Strings are composed of five items or fewer, but most typically have two to three items. Items are, typically, one or two words long, with the longest item the three-word unit of force. Mathematical items are all single symbols composed of an alphabetical letter, aside from $F 1$, the only letter-number combination. Overall, force and weight are expressed as simple lexical repetitions and as the simple mathematical synonyms $W$ or F1. Force, weight, and spring are also realized in small collocation groupings of simple, quasi-technical terms.

Garnet. The lexical string analysis, presented in Appendix G and summarized in Table 9, below, reveals this text's area of focus as friction and force. These topics are usually developed through the classification relationships of repetition and hyponymy and through relationships of collocation. Repetition strings are most common: friction is repeated 14 times across the four paragraphs while force is repeated nine times across three paragraphs. In a smaller role, five relationships of hyponymy, or class/sub-class relationships, are developed; all are shallow taxono- 
mies such as the following: scalar value: coefficient of friction and force: friction and materials: rubber tyre, road, piston, cylinder. One tie of meronymy, or a whole-part relationship, describes friction and offers the most complex example of topic development. The coefficient of friction is designated with its parts normal force $N$ and force of resistance $R$, the parts expressed lexically and mathematically. Technical collocations are limited and show the three terms coefficient of friction, scalar value, and static friction force while the mathematical collocation includes four items: $f s=\mu s N, \mu, N, R$. Other collocations are non-technical, such as tyre: road or speed: motion.

Strings range from two items in length up to the one 14-item repetition string; most nonrepetition strings, however, tend to be composed of two or three items. Lexical items are typically one to three words long while mathematical items are normally expressed by a single graphical item, aside from the one complex, multi-term mathematical formula. Overall, friction and force are realized as lexical repetitions and presented in shallow taxonomies and small collocation groupings that include mostly simple mathematical terms.

Hibbeler: Forces. The lexcial relations analysis is summarized in Table 9, below, and reveals that lexical strings are most often built around the topics mass, forces, and springs. These topics are typically presented in classification relationships of repetition, hyponymy, and meronymy and through collocation relationships. Repetition strings are most common. Spring, for example, is repeated eight times in one paragraph. Although strings are usually shorter, each paragraph shows at least three repetition strings, even the short, two-sentence "Mass" paragraph. In a somewhat smaller role, six hyponymy relationships develop the main topics. One, a multilevel taxonomy, develops forces this way: 
forces: contact forces, distance forces

distance forces: gravitational, electrical, magnetic.

Other taxonomies show, more typically, shallow topic development, such as measure:

mass, velocity. At least four meronymy relationships also develop topics. The spring is expressed in terms of its length (deformed or undeformed), its stiffness, and its position. Force is characterized by the complex, three-item magnitude, direction, and point of application. Other meronymy relationships are less complex, composed of just two lexical items. The most significant technical collocation is built around linearly elastic springs, itself a technical term, and includes spring constant, stiffness $k$, deformed length, undeformed length, and unloaded position. Interestingly, when non-technical terms, such as elasticity, are used to describe springs, the non-technical term is enclosed in distancing quotes: "elasticity". Distancing quotes are used this way three times in this paragraph. Simple and complex mathematical terms are also collocated: $k, s, l, F$, and the formula $F=k s$. The formula is also worked out in an example, $(500 \mathrm{~N} / \mathrm{m})(0.6 \mathrm{~m}-0.4 \mathrm{~m})=100 \mathrm{~N}$, which could be said to collocate with the other mathematical terms. This formula and its working-out could reasonably be considered a hyponymy relationship, with the formula representing the superordinate term.

On average, strings tend to be composed of two to five items, although one collocation and two repetition strings are longer. Items in a string are typically one to three words long. Mathematical items range from single alphabetic symbols to complex expressions and workings. The topics mass, forces, and springs are expressed through extensive repetition, shallow and deep taxonomies, relationships of hyponymy, and in extensive technical collocations. 
Hibbeler: Friction. Lexical strings are most often built around the topics force and friction, as shown in the lexical relations analysis, presented in Appendix G and summarized in Table 9 , below. The topics are most usually presented in classification relationships of synonymy and repetition and in collocation relationships. Friction, for example, is repeated nine times across two paragraphs while force is repeated 15 times in the same paragraphs. Synonymy constructs at least 12 bonds across the three paragraphs, with the most common pattern adding a mathematical meaning dimension to a lexical item. Typical examples include: frictional force and $\Delta F n$; limiting static frictional force and $F s$; and uniform weight and $W$. The mathematical item may be a single symbol, such as $W$, or a compound term such as $\Delta F n$. Collocation relationships bringing together technical words or mathematical symbols also express the main topics. A good example is limiting static frictional force, constant of proportionality, coefficient of static friction, and angle of static friction. The mathematical string is made up of $F s=\mu s N, \mu s, F s, N$, $R$.

Lexical strings range up to 13 items long in the case of repetition, but most are made up of four items or fewer. Items are, typically, one to three words long although several are as long as four words. Mathematical items range from simple alphabetic symbols to complex expressions, including a formula. The topics force and friction are presented and expressed in extensive repetition strings, in simple and complex multimodal synonymy groupings, and through extensive technical collocations.

Summary. The 'forces' texts, Oxford and Hibbeler: Forces, and the 'friction' texts, Garnet and Hibbeler: Friction, generally share the same main topics but significant differences in topic complexity are demonstrated (see the lexical relations summary, Table 9). The 'forces' texts 
are both concerned with force and springs while the Oxford text also shows weight as a major topic and Hibbeler shows mass. The 'friction' texts share the topics friction and force. All texts reveal repetition as a major relationship among words. Since these words are frequently the main topic words, repetition can be said to be an important method of topic presentation. Similarities, however, seem to end here with further analysis revealing important differences. Both Hibbeler samples show highly specified topics, extensive technical collocations, and include complex mathematical expressions. For example, Hibbeler: Forces describes forces in a two-level taxonomy: forces: contact forces, distance forces - distance forces: gravitational, electrical, magnetic. In contrast, the most complex descriptions of force in Oxford are two-word phrases with a simple mathematical symbol: for example, buoyancy force, $B$. The most complex rendering of springs in Oxford is the three-term technical group about measurement: spring balance, calibrated, newtons. In Hibbeler, springs are highly specified across four technical parameters: linearly elastic spring, spring constant, stiffness $k$, deformed and undeformed length, unloaded position. Mathematical symbols in Oxford are most often single alphabetic symbols, such as $B$ for buoyancy force. The single multiple term, $F 1$, representing upward force, simply describes another type of force. Hibbeler uses a math formula to determine the magnitude of force needed to stretch a spring: $F=k s$ and works out that formula as a complex mathematical example embedded in a written paragraph. 
Table 9

Lexical relations summary

\begin{tabular}{|c|c|c|c|c|}
\hline Feature & Oxford & Garnet & Hibbeler: "Forces" & $\begin{array}{l}\text { Hibbeler: } \\
\text { "Friction" }\end{array}$ \\
\hline $\begin{array}{l}\text { Head items of longest } \\
\text { strings (no. of words in } \\
\text { strings) }\end{array}$ & $\begin{array}{l}\text { force }(5,4) \\
\text { weight }(4,3) \\
\text { springs }(5)\end{array}$ & $\begin{array}{l}\text { friction (6) } \\
\text { force (4) }\end{array}$ & $\begin{array}{l}\text { force }(5,7) \\
\text { springs }(8) \\
\text { length }(4)\end{array}$ & $\begin{array}{l}\text { force }(9,4) \\
\text { friction }(6) \\
\text { block }(6)\end{array}$ \\
\hline $\begin{array}{l}\text { Predominant type of } \\
\text { lexical relation (no. of } \\
\text { strings) }\end{array}$ & $\begin{array}{l}\text { synonymy (11) } \\
\text { repetition (10) }\end{array}$ & $\begin{array}{l}\text { repetition (11) } \\
\text { shallow taxono- } \\
\text { mies }(5)\end{array}$ & $\begin{array}{l}\text { repetition }(10) \\
\text { deep \& shallow } \\
\text { taxonomies (6) } \\
\text { meronymy (4) }\end{array}$ & $\begin{array}{l}\text { synonymy (12) } \\
\text { repetition (11) }\end{array}$ \\
\hline $\begin{array}{l}\text { Typical string length, } \\
\text { by item (excluding } \\
\text { repetitions) }\end{array}$ & $2-3$ & $2 \cdot 3$ & $2-5$ & $2-4$ \\
\hline $\begin{array}{l}\text { Typical word count per } \\
\text { item }\end{array}$ & $1-2$ & $1-2$ & $1-3$ & $1-3$ \\
\hline Typical math items & single symbols & $\begin{array}{l}\text { single symbols \& } \\
\text { one expression }\end{array}$ & $\begin{array}{l}\text { multiple symbols } \\
\text { \& expressions }\end{array}$ & $\begin{array}{l}\text { multiple sym- } \\
\text { bols \& expres- } \\
\text { sions }\end{array}$ \\
\hline Technical collocations & $\begin{array}{l}\text { mostly quasi- } \\
\text { technical }\end{array}$ & limited & extensive & extensive \\
\hline
\end{tabular}

The longest strings in the two 'friction' texts, Hibbeler: Friction and Garnet, reveal fric-

tion and force as major topics. In Garnet, these topics are developed primarily through repetition: four repetition strings present friction, the longest containing six repetitions, while three present force, the longest with four repetitions. Friction and force are also part of three shallow, twoword taxonomies, expressed simply as force: friction. The technical collocation coefficient of friction, scalar value, and static friction force plus four mathematical items, three simple and one complex, further represent friction and force. In contrast, Hibbeler: Friction develops force in a nine-item repetition chain, plus two smaller repetition strings, and in six synonymy pairings matching names of forces to complex mathematical symbols, such as reactive force and $\Delta R n$. Ten mathematical items, including five complex symbols and a complex formula, plus the complex 
technical collocation limiting static frictional force, constant of proportionality, coefficient of static friction, and angle of static friction also represent the main topics.

The complex expression of topic in both Hibbeler samples is evidenced in the overall tendency to longer strings and in the longer word count per string item as well as in longer technical collocation strings and math items composed of multiple symbols and full expressions. Also, the head items of the longest strings in the EAP texts and in the Hibbeler texts contrast noticeably. For example, of the two 'forces' texts, Oxford's head words, force, weight, springs, express two general concepts, while Hibbeler's head words, force, springs, and length, show a focusing of attention to concrete characteristics. A similar pattern is observed in the 'friction' texts. Furthermore, while all texts evidence lexical relationships of repetition, relationships of synonymy, meronymy, and taxonomy also work to develop topic complexity in the Hibbeler samples. Extensive use of synonymy connects lexical terms to corresponding mathematical terms, meronymies distinguish topics from their parts, and taxonomies build classification schemes. This development of taxonomies in topic description is typical of technical language (Eggins, p. 109).

\section{Visual Representations of Subject Matter}

\section{Representation analysis}

The visual representation analysis is the visual expression of the verbal transitivity analysis and is thus concerned with representations of experiential reality. Like transitivity, the focal point of this analysis is the process, or the represented activities, and the participants associated with each process type. Processes are either narrative or conceptual in structure. Conceptual structures expresss reality in terms of 'being' and construct Carriers and Attributes as participants. Narrative structures express reality as a matter of 'doing' and construct their partici- 
pants as Actors, or the doers of actions, and Goals, or the recipients of the action. Circumstantial elements add experiential detail in terms of manner, quality, and temporal space and time.

Oxford. The eight visual elements analyzed here include seven diagrams and one drawing. The analysis is summarized by structure, process, participant, and circumstance in Table 10 below. Of the eight visuals, four are structurally narrative and four are conceptual. In other words, half the visuals represent reality in terms of 'doing' and the other half in terms of 'being'. The narrative structures are all non-transactional actions, actions that are not 'done to' another entity since all depict action as an arrow emanating from a body but not directed towards another body. In all four cases, the presence of the arrows establishes both processes of action and participants. In the case of the boat diagram, for example, two arrows show directionality, and thus action, with the arrowheads pointing away from the boat; they show participant status because they are labelled B and W, or buoyancy and weight, respectively. In total, the participants in the non-transactional actions number 15 Actors across the four diagrams. The four conceptual structures, three diagrams and one drawing, are exhaustive analytical processes which means that they show no action but represent their participants by depicting some aspect of their being. In the case of the three diagrams, all are shown with topological accuracy which establishes logical spatial relationships (but not spatially accurate relationships). For example, in the spring balance diagram, the block is shown as hanging from a hook at the bottom of the balance, establishing the spatial connection between the two participant Carriers, the balance and the block. The other two conceptual diagrams also establish two Carriers each as participants, although the relationships between the participant Carriers seem so obvious as to be overlooked. For example, the ship diagram is composed of two visual elements: the ship and a wavy line representing water. 
The most straightforward reading of this diagram is to interpret the ship as a Carrier and the water as a Circumstance of Setting. This is the analysis that I first proposed for this diagram but a problem developed. The problem is that the ship is so highly abstracted that it possesses no attributes; this reading would pointlessly establish the ship as a Carrier of no Attributes. In searching for another possible interpretation, I looked at the accompanying verbal question that directs readers to the ship visual: Why doesn't the ship sink? The wavy water line is clearly establishing the ship as not sinking; therefore, the process this diagram is asserting must be the un-sinking relationship between the ship and the water. This process is the topological process that shows spatial relationships between two participants, in this case, the ship and the water. This visual relationship as represented, though, is so elementary as to seem out of place in a tertiary-level textbook. In the narrative diagram of the same ship in water, the water is represented as the more straightforward Circumstance of Setting.

The drawing of a wrench is shown with topographical accuracy, establishing accurate physical spatial relationships in the participant. The wrench is related in its entirety in a partwhole structure showing two lengths, D1 and D2, and two points on the lengths, F1 and F2. The wrench, then, is the Carrier and the two lengths and two points are Possessive Attributes. Overall, of the 26 participants established in the eight visuals, 12 are expressed mathematically: seven as single mathematical symbols such as B or $\mathbf{W}$, and five as more complex symbols composed of two items such as D1 or D2. Collectively, all eight visuals establish three circumstantial elements. All three are abstracted settings such as the wavy line that represents water in the narrative boat diagram. 
Table 10

Oxford representation summary

\begin{tabular}{|c|c|c|c|c|}
\hline Visual & Structure & Process & Participant & Circumstance \\
\hline Diagram: boat & Conceptual & $\begin{array}{l}\text { exhaustive ana- } \\
\text { lytical, topologi- } \\
\text { cal accuracy }\end{array}$ & $\begin{array}{l}\text { - } 2 \text { participants: } \\
\text {. } 2 \text { Carriers: ship, water }\end{array}$ & none \\
\hline $\begin{array}{l}\text { Diagram: } \\
\text { spring balance }\end{array}$ & Conceptual & $\begin{array}{l}\text { exhaustive ana- } \\
\text { lytical, topologi- } \\
\text { cal accuracy }\end{array}$ & $\begin{array}{l}\text { - } 2 \text { participants: } \\
\text { - } 2 \text { Carriers: spring bal- } \\
\text { ance, block }\end{array}$ & none \\
\hline Diagram: box & Conceptual & $\begin{array}{l}\text { exhaustive ana- } \\
\text { lytical, topologi- } \\
\text { cal accuracy }\end{array}$ & $\begin{array}{l}2 \text { participants: } \\
\quad \text { - } 2 \text { Carriers: box, slope }\end{array}$ & none \\
\hline Diagram: boat & Narrative & $\begin{array}{l}\text { non-transactional } \\
\text { action }\end{array}$ & $\begin{array}{l}\text { - } 3 \text { participants: } \\
\text {. } 3 \text { Actors: B, W, boat }\end{array}$ & $\begin{array}{l}\text { abstracted water: } \\
\text { wavy line }\end{array}$ \\
\hline $\begin{array}{l}\text { Diagram: } \\
\text { spring balance }\end{array}$ & Narrative & $\begin{array}{l}\text { non-transactional } \\
\text { action }\end{array}$ & $\begin{array}{l}\text { - } 3 \text { participants: } \\
\text { - } 3 \text { Actors: } \mathrm{F} 1, \mathrm{~W} \text {, spring } \\
\text { balance }\end{array}$ & none \\
\hline Diagram: box & Narrative & $\begin{array}{l}\text { non-transactional } \\
\text { action }\end{array}$ & $\begin{array}{l}\text { - } 5 \text { participants: } \\
\text { - } 5 \text { Actors: } R, \mathrm{~W}, \text { resul- } \\
\text { tant, friction, box }\end{array}$ & $\begin{array}{l}\text { abstracted slope: } \\
\text { angled line }\end{array}$ \\
\hline Diagram: block & Narrative & $\begin{array}{l}\text { non-transactional } \\
\text { action }\end{array}$ & $\begin{array}{l}\text { - } 4 \text { participants: } \\
\text { - } 4 \text { Actors: F, P, block, } \\
\text { balance }\end{array}$ & $\begin{array}{l}\text { abstracted table- } \\
\text { top: rectangle }\end{array}$ \\
\hline Drawing: tool & Conceptual & $\begin{array}{l}\text { exhaustive ana- } \\
\text { lytical, topog- } \\
\text { raphical accuracy }\end{array}$ & $\begin{array}{l}\text { - } 5 \text { participants: } \\
\text { - } 1 \text { Carrier: wrench } \\
\text { - } 4 \text { Possessive Attrib- } \\
\text { utes: D1, D2, F1, F2 }\end{array}$ & none \\
\hline
\end{tabular}

Garnet. The five visual elements in this analysis include two graphs and three photographs; Table 11 below summarizes the analysis by structure, process, participant, and circumstance. The three photographs are conceptual structures while the two graphs are narrative; thus, visual representations of reality slightly favour expressions of 'being' rather than expressions of 'doing'. The conceptual structures all show topographical accuracy but two are exhaustive, showing the entire participant, and one is inclusive, including only a part of the participant. All 
three establish two participants each, the Carrier, which differs in each case, and the Possessive Attribute, friction, which is the same in each case. This Attribute must be inferred from textual elements as no visual indication is provided.

Table 11

Garnet representation summary

\begin{tabular}{|c|c|c|c|c|}
\hline Visual & Structure & Process & Participant & Circumstance \\
\hline $\begin{array}{l}\text { Graph: } \\
\text { Figure } 1\end{array}$ & Narrative & $\begin{array}{l}\text { unidirectional } \\
\text { transactional action } \\
\text { (and } 1 \text { embedded } \\
\text { unidirectional } \\
\text { transactional proc- } \\
\text { ess) }\end{array}$ & $\begin{array}{l}\text { - } 4 \text { participants: } \\
\text { - } 2 \text { Actors: friction force, ap- } \\
\text { plied force } \\
\text { - } 2 \text { Goals: static friction, ki- } \\
\text { netic friction } \\
\text { - } 2 \text { embedded participants: } \\
\text { - } 1 \text { Actor: static friction } \\
\text { - } 1 \text { Goal: point of motion }\end{array}$ & none \\
\hline $\begin{array}{l}\text { Graph: } \\
\text { Figure } 2\end{array}$ & Narrative & $\begin{array}{l}\text { unidirectional } \\
\text { transactional action }\end{array}$ & $\begin{array}{l}\text { - } 5 \text { participants: } \\
\text { - } 2 \text { Actors: friction force, } \\
\text { normal force } \\
\text { - } 3 \text { Goals: rubber on asphalt, } \\
\text { steel on steel, ice on steel }\end{array}$ & $\begin{array}{l}\text { Means: } \mu=y / \\
x, x, y\end{array}$ \\
\hline $\begin{array}{l}\text { Photo: } \\
\text { top left }\end{array}$ & Conceptual & $\begin{array}{l}\text { exhaustive analyti- } \\
\text { cal, topographical } \\
\text { accuracy }\end{array}$ & $\begin{array}{l}\text { - } 2 \text { participants: } \\
\text { - } 1 \text { Carrier: steel ring } \\
\text { - } 1 \text { Possessive Attribute: fric- } \\
\text { tion }\end{array}$ & Setting: surface \\
\hline $\begin{array}{l}\text { Photo: } \\
\text { top right }\end{array}$ & Conceptual & $\begin{array}{l}\text { exhaustive analyti- } \\
\text { cal, topographical } \\
\text { accuracy }\end{array}$ & $\begin{array}{l}\text { - } 2 \text { participants: } \\
\text { - } 1 \text { Carrier: steel plate } \\
\text { - } 1 \text { Possessive Attribute: fric- } \\
\text { tion }\end{array}$ & Setting: surface \\
\hline $\begin{array}{l}\text { Photo: } \\
\text { bottom }\end{array}$ & Conceptual & $\begin{array}{l}\text { inclusive analytical, } \\
\text { topographical accu- } \\
\text { racy }\end{array}$ & $\begin{array}{l}\text { - } 2 \text { participants: } \\
\text { - } 1 \text { Carrier: machine } \\
\text { - } 1 \text { Possessive Attribute: fric- } \\
\text { tion }\end{array}$ & none \\
\hline
\end{tabular}

The two narrative structures, the graphs, both relate unidirectional transactional actions, or actions that emanate from one participant, an Actor, towards another, a Goal. In these cases, the Actors are realized as the $\mathrm{X}$-axis and $\mathrm{Y}$-axis. The Goals are the diagonal lines on the graph that represent the angle of convergence of the axes. The first graph represents two types of fric- 
tion as Goals while, in the second graph, the Goals are three different coefficients of friction. The first graph also relates an embedded process, another unidirectional transactional action, with one Actor and one Goal, thus bringing the total number of Actors to five and Goals to six. Circumstances are realized as two Settings, both surfaces, and one Means, the tool or method used to derive the ice on steel coefficient of friction. In this case, the tool is the mathematical expression $\mu=y / x$. The tool is further specified as the line $x$ and the line $y$.

Hibbeler: Forces. Six visual elements comprise this analysis, three photographs and three diagrams, with results summarized by structure, process, participant, and circumstance in Table 12, below. Of the six visuals, five show narrative structures while one is conceptual. The five narrative structures include three unidirectional transactional actions and two nontransactional actions. The three unidirectional processes are all represented in photographs, two of which are associated with matching diagrams. The first photograph, a full-colour representation opening the chapter, foregrounds the unidirectional process just mentioned and shows one Actor, a crane boom, and a shipping container, the Goal towards which the action is directed. Several minor narrative structures are also related through the boatyard Setting but as these processes and participants are very small and clearly backgrounded by their size, they will be omitted in this analysis. The other two photographs are each laid out on the page next to two matching diagrams such that the diagram 'translates' the photograph's unidirectional process that shows an action directed from one participant towards another into a non-transactional action that simply expresses the action of a participant. The photograph and its matching diagram are positioned in such a way that the spatial placement of each photographic participant is mirrored in the diagram. In this way, the concrete photographic representation is recontextualized into the abstract 
diagrammatic representation. In the first photograph/diagram pair, the photograph's participants, Actor and Goal, are a cable and a bucket; the unidirectional action shows the cable supporting or holding the bucket. The diagram modifies the process to a non-transactional action, an action not directed towards another participant and, in so doing, transforms the cable Actor and the bucket Goal into three Actors: T, or the force of the cable, W, or the bucket's weight, and the bucket itself. The second photograph/diagram set represents a similar movement from unidirectional process to non-transactional process. In this movement, the unidirectional participants, three Actors, all cables, and one Goal, a spool, are transformed into four non-transactional Actors: a ring and three forces. It is through this representational recontextualization that engineering's freebody diagram isolates a bucket, for example, so "the unknown cable force T becomes "exposed" (Hibbeler, 2007, p. 85) thereby accounting for forces which may not be readily apparent in the more representational photograph.

The conceptual structure, a diagram of three springs, shows an exhaustive analytical process with topographical accuracy. The diagram is analytical in that it relates the whole to its parts; it is exhaustive since it shows the entire whole and not just a section or portion of the whole; and it is topographical because it relates accurate spatial relationships among the parts. In this case, the drawing represents three springs which are the Carriers of eight Possessive Attributes, characteristics of the spring that we may recall from the verbal discussion: its stiffness, its deformed and undeformed length, and its deformed and undeformed distance (since not each attribute is realized by each spring, the total number of attributes is eight and not 15). Seven of the Attributes are related as mathematical expressions which are composed of the attribute's mathematical symbol, for example $l$ identifies deformed length, and its mathematical instantiation as a 
measurement, in this case $0.2 \mathrm{~m}$. One attribute is related only as its mathematical symbol.

Mathematical symbols, related as single alphabetic letters, are also used to name five forces, four cables, and one ring in the previously described photographs and diagrams, for a total of seven complexly identified mathematical participants and 11 simple mathematical participants. Across all six visual representations in this analysis, four Circumstances of Setting are realized. Of these, three are fully representational, such as the boatyard, while one is an abstracted ceiling.

Table 12

Hibbeler: Forces representation summary

\begin{tabular}{|c|c|c|c|c|}
\hline Visual & Structure & Process & Participant & Circumstance \\
\hline Photo: boat & Narrative & $\begin{array}{l}\text { unidirectional transac- } \\
\text { tional action }\end{array}$ & $\begin{array}{l}\text { - } 2 \text { participants: } \\
\text { - } 1 \text { Actor: crane or } \\
\text { cable } \\
\text { - } 1 \text { Goal: shipping } \\
\text { container }\end{array}$ & $\begin{array}{l}\text { Setting: boatyard } \\
\text { (with back- } \\
\text { grounded narrative } \\
\text { structures) }\end{array}$ \\
\hline $\begin{array}{l}\text { Diagram: } \\
\text { spring }\end{array}$ & Conceptual & $\begin{array}{l}\text { exhaustive analytical, to- } \\
\text { pographical accuracy }\end{array}$ & $\begin{array}{l}\text { - } 8 \text { participants: } \\
\text { - } 3 \text { Carriers: } \\
\text { springs } \\
\text { - } 8 \text { Attributes: } \\
\text { mathematical }\end{array}$ & $\begin{array}{l}\text { Setting: ceiling, } \\
\text { abstracted (with } 2 \\
\text { embedded non- } \\
\text { transactional proc- } \\
\text { esses) }\end{array}$ \\
\hline Photo: A & Narrative & $\begin{array}{l}\text { unidirectional transac- } \\
\text { tional action }\end{array}$ & $\begin{array}{l}\text { - } 2 \text { participants: } \\
\text { - } 1 \text { Actor: cable } \\
\text { - } 1 \text { Goal: bucket }\end{array}$ & Setting: sky \\
\hline $\begin{array}{l}\text { Diagram: } \\
\text { with A }\end{array}$ & Narrative & non-transactional action & $\begin{array}{l}\text { - } 3 \text { participants: } \\
\text { - } 3 \text { Actors: bucket, } \\
\text { T, W }\end{array}$ & none \\
\hline Photo: B & Narrative & $\begin{array}{l}\text { unidirectional transac- } \\
\text { tional action }\end{array}$ & $\begin{array}{l}4 \text { participants: } \\
\text { - } 3 \text { Actors: the } \\
\text { cables DA, AB, } \\
\text { AC } \\
\text { - } 1 \text { Goal: spool }\end{array}$ & $\begin{array}{l}\text { Setting: construc- } \\
\text { tion yard }\end{array}$ \\
\hline $\begin{array}{l}\text { Diagram: } \\
\text { with B }\end{array}$ & Narrative & non-transactional action & $\begin{array}{l}4 \text { participants: } \\
\text { - } 4 \text { Actors: the } \\
\text { ring } A, \text { the } \\
\text { forces } W, T b, T c\end{array}$ & none \\
\hline
\end{tabular}


Hibbeler: Friction. The six visual representations that comprise this analysis include four diagrams and two photographs with results summarized by structure, process, participant, and circumstance in Table 13, below. The two photographs open the chapter; a close-up shot of a bicycle's brake system is a full-page non-transactional action with one Actor, the brake system. A smaller photograph relates a unidirectional action with a partially visible Actor, a person, sharpening a metal blade, the Goal. The remaining four diagrams are part of a set of five used to illustrate aspects of the theory of dry friction (only four are analyzed here to maintain correspondence with the verbal analysis). The opening diagram in the set is a non-transactional action, relating three Actors: a block and two forces, $\mathrm{W}$ and $\mathrm{P}$. The remaining diagrams all represent the same block but highlight different 'goings-on' as unidirectional transactional actions. In this way, the original three Actors are complemented with additional Actors and new Goals such that the total number of participants across the four diagrams is 21 . All participants, aside from the block and the surfaces, are related as mathematical symbols, either single alphabetic letters, such as W for weight, or as more complex terms such as $\Delta \mathrm{Fn}$ for the distributed friction force. Diagrams $\mathrm{B}$ and C also relate their Goals in multiples to add further experiential information. Each Goal in Diagram $\mathrm{C}$ is mathematically identified three times to show a recurrent pattern while both Goals in Diagram B are represented with multiple arrowheads in order to show continuous distribution. As well, Diagram E shows three embedded processes, each a bidirectional transactional action which connects two Actors and, in these cases, produces a third Actor. With the inclusion of the embedded Actors, the total number of participants across the four diagrams is 24. Circumstances are related five times across all visual representations. Setting is shown three times while Means is shown twice. In Diagram E, the Means is identified lexically as Impending motion. 
Table 13

Hibbeler: Friction representation summary

\begin{tabular}{|c|c|c|c|c|}
\hline Visual & Structure & Process & Participant & Circumstance \\
\hline $\begin{array}{l}\text { Photo: brake } \\
\text { system }\end{array}$ & Narrative & non-transactional action & $\begin{array}{l}\text { - } 1 \text { participant: } \\
\text { - } 1 \text { Actor: brake system }\end{array}$ & none \\
\hline $\begin{array}{l}\text { Photo: } \\
\text { grinder }\end{array}$ & Narrative & $\begin{array}{l}\text { unidirectional transac- } \\
\text { tional action }\end{array}$ & $\begin{array}{l}\text { - } 2 \text { participants: } \\
\text { - } 1 \text { Actor: person } \\
\text { - } 1 \text { Goal: metal blade }\end{array}$ & $\begin{array}{l}\text { Means: } \\
\text { grinder } \\
\text { Setting: work- } \\
\text { shop }\end{array}$ \\
\hline Diagram: A & Narrative & non-transactional action & $\begin{array}{l}3 \text { participants: } \\
\text { - } 3 \text { Actors: block, W, P }\end{array}$ & $\begin{array}{l}\text { Setting: rough } \\
\text { surface }\end{array}$ \\
\hline Diagram: $\mathbf{B}$ & Narrative & $\begin{array}{l}\text { unidirectional transac- } \\
\text { tional action }\end{array}$ & $\begin{array}{l}\text { - } 5 \text { participants: } \\
\text { - } 3 \text { Actors: block, W, P } \\
\text { - } 2 \text { Goals: } \Delta \mathrm{Nn}, \Delta \mathrm{Fn}\end{array}$ & none \\
\hline Diagram: $\mathrm{C}$ & Narrative & $\begin{array}{l}\text { unidirectional transac- } \\
\text { tional action }\end{array}$ & $\begin{array}{l}\text { - } 5 \text { participants: } \\
\text { - } 2 \text { Actors: floor surface, } \\
\text { block surface } \\
\text { - } 3 \text { Goals: } \Delta \mathrm{F}, \Delta \mathrm{N}, \Delta \mathrm{R}\end{array}$ & $\begin{array}{l}\text { Setting: con- } \\
\text { tacting sur- } \\
\text { faces }\end{array}$ \\
\hline Diagram: E & Narrative & $\begin{array}{l}\text { unidirectional transac- } \\
\text { tional action (with } 3 \mathrm{em}- \\
\text { bedded bidirectional } \\
\text { transactional actions) }\end{array}$ & $\begin{array}{l}\text { - } 8 \text { participants: } \\
\text { - } 7 \text { Actors: block, surface, } \\
\text { W, P, F, N, R } \\
\text { - } 1 \text { Goal: equilibrium } \\
\text { - } 3 \text { embedded participants: } \\
\text { - } 3 \text { Actors: } x, h \text {, angle } ø\end{array}$ & $\begin{array}{l}\text { Means: im- } \\
\text { pending mo- } \\
\text { tion }\end{array}$ \\
\hline
\end{tabular}

Summary. The findings of the Representation analysis are summarized by structure, process, participant, and mathematical participant for each text in Table 14 below. The summary reveals that visuals in the texts are expressed differently and thus present different representations of reality. The Oxford and Garnet visuals, with almost equal proportions of narrative and conceptual structures, represent reality more or less equally in terms of 'doing' and 'being'. The Hibbeler visuals relate reality more as a matter of 'doing' than as a matter of 'being'. The Hibbeler visuals also show higher proportions of unidirectional transactional actions, actions that implicate more than 1 participant or entity in the doing of the process. The overall number of 
participants in Hibbeler is higher, too, both proportionally and in real numbers. Hibbeler: Forces shows 27 participants across six visuals while Hibbeler: Friction has 27 participants in six visuals. Garnet shows 17 participants in five visuals, while Oxford has 24 participants in eight visuals. These higher numbers and proportions of participants in Hibbeler establish well-populated visual environments concerned with the interactions of participants in environments specified by those participants. For example, in the Hibbeler: Friction Diagram C, 11 participants illustrate the development of three classes of friction in an environment specified by protuberances or contacting bumpy surfaces. In contrast, the most populated visuals in Oxford and Garnet contain five participants. In terms of expression of participant types, the Hibbeler visuals are distinctive. In Hibbeler: Forces, participant expression is realized in a pattern combining a high proportion of Actors, an attention to Attributes, and a representation of Goals. In Friction that realization is in the combined high proportion of Actors and high representation of Goals. The other two texts do not exhibit either of these patterns. Oxford fails to represent Goals while Garnet shows a considerably lower proportion of Actors to other participants. Participants are also much more frequently expressed as mathematical terms in Hibbeler. For example, of the 33 participants in Hibbeler: Friction, 23 are expressed mathematically, 12 in simple, one character terms such as W for weight, and 11 in complex terms such as $\Delta N_{1}$ for distributed normal force. Hibbeler: Forces names 18 of its 27 participants mathematically, in both simple and complex terms. All seven of the complex terms are mathematical expressions involving a process, for example $k=500 \mathrm{~N} / \mathrm{m}$. Garnet mathematicalizes three participants, twice simply as $x$ and $y$ and once complexly as $\mu=y /$ $x$. Oxford names 12 participants mathematically, most in simple terms such as $\mathrm{W}$ for weight, but several in slightly more complex, two character terms such as $F_{1}$ for upward force. This differ- 
ence in the mathematical expression of visual participants is one of the most noticeable differences between visual elements in Hibbeler and in Oxford and Garnet.

Table 14

Representation summary, by text

\begin{tabular}{|c|c|c|c|c|c|}
\hline \multicolumn{2}{|c|}{ Feature } & Oxford & Garnet & $\begin{array}{l}\text { Hibbeler: } \\
\text { Forces }\end{array}$ & $\begin{array}{l}\text { Hibbeler: } \\
\text { Friction }\end{array}$ \\
\hline \multicolumn{2}{|l|}{ Number of visuals } & 8 & 5 & 6 & 6 \\
\hline \multirow[t]{3}{*}{ Narrative processes } & non-transactional & 4 & $\mathbf{0}$ & 2 & 2 \\
\hline & unidirectional & 0 & 2 & 3 & 4 \\
\hline & Total & 4 & 2 & 5 & 6 \\
\hline \multirow[t]{3}{*}{$\begin{array}{l}\text { Conceptual proc- } \\
\text { esses }\end{array}$} & $\begin{array}{l}\text { topographical accu- } \\
\text { racy }\end{array}$ & 1 & 3 & 1 & 0 \\
\hline & $\begin{array}{l}\text { topological accu- } \\
\text { racy }\end{array}$ & 3 & 0 & 0 & 0 \\
\hline & Total & 4 & 3 & 1 & $\mathbf{0}$ \\
\hline \multirow[t]{5}{*}{ Participants } & actors & 15 & 5 & 13 & 20 \\
\hline & goals & 0 & 6 & 3 & 7 \\
\hline & carriers & 5 & 3 & 3 & 0 \\
\hline & attributes & 4 & 3 & 8 & 0 \\
\hline & Total & 24 & 17 & 27 & 33 \\
\hline \multirow{3}{*}{$\begin{array}{l}\text { Participants ex- } \\
\text { pressed mathemati- } \\
\text { cally }\end{array}$} & simple & 7 & 2 & 11 & 12 \\
\hline & complex & 5 & 1 & 7 & 11 \\
\hline & Total & 12 & 3 & 18 & 23 \\
\hline \multicolumn{2}{|l|}{ Embedded Processes } & 0 & 1 & 2 & 3 \\
\hline
\end{tabular}

\section{Coding orientation analysis}

Coding orientations describe how texts are coded for interpretation with four broad classifications describing the dominant interpretations. The four interpretations are distinguished as 
technological, sensory, abstract, and naturalistic orientations. In this analysis, each orientation is determined by reference to five visual criteria - colour saturation, colour differentiation, colour modulation, context, and representation - and distinguished by the scalar measurement of each criterion: in this analysis, high, medium, or low. In general, technological and abstract codings fall towards the lower end of the scales, showing reduced colour, context, and detail. The abstract coding is differentiated by its reduction of the individual to the general. Sensory codings fall towards the higher end of the scales with intense use of colour, often highly contextualized settings, and so on, while naturalistic codings tend to fall somewhere in between medium and high. Coding orientations are commonly used to describe the believability of visual information; however, the orientations may also be used, as here, to determine the type of reality a text wishes to encode. For this analysis, the Hibbeler texts are treated as one since the orientation is consistent throughout the text.

Oxford. The Oxford text shows a technological coding orientation since most criteria fall at the lower end of the continuum. Colour saturation, or the intensity of a colour, is uniformly medium-low; that is, colours are mostly pale or light-toned. Colour differentiation, or the variety of colour, is low: blue is the only colour used other than black. Colour modulation measures the range of tones within one colour; with two tones of blue and a range of grey tones, this measurement falls into the medium-low range. The context scale describes the level of detail in visual backgrounds. Here, that level is low: very little to no background detail is shown. The most contextualized visual shows a diagram of a ship and represents the water in which it sits with an abstracted wavy line. In this text, most drawings are almost fully abstracted, such as the ship, while one drawing of a wrench shows an almost naturalistic representation. Because the text represents 
its visuals in both manners, and with a greater proportion of abstracted visuals, this criterion is rated medium-low.

Garnet. The coding orientation in the Garnet text is technological. Colour saturation is medium-low since colours are usually pale, aside from one deeper blue tone. Colour differentiation is limited, and thus placed at medium-low, as the text relies primarily on three colours: blue, yellow, and beige. Of these three colours, only the blue is modulated, showing variety in shades, so this criterion also falls at medium-low on the scale. Three photographs, all extreme close-ups, omit contextual detail, as do tear-outs of note-paper, meant to represent student work, thus accounting for the low context measurement. Representation in this text is variable: the two graphs and the six tear-out notes are abstractions while the three photographs are almost hyper-realistic. This dual representation of visuals, with a higher proportion of abstracted items, explains the medium-low rating.

Hibbeler. A naturalistic orientation describes the coding of the Hibbeler text. Colour saturation, differentiation, and modulation are all medium-high. Colours show a true-to-life degree of saturation, with both paler and deeper tones in photographs, drawings, and layout elements such as borders and banners. The range of colours, or colour differentiation, is also realistic: photographs show scenes from working life in naturalistic tones, and other visual elements, such as diagrams, borders, and banners, use a range of natural-world colours: blues, greens, greys, oranges, and yellows. The photographs, by virtue of their realism, show colour modulation, or range in shades, as do the drawings and diagrams which are usually shaded to show shape, tension, or some other relevant characteristic. The context criterion, or background detail, varies in this text. The free-body diagrams, as abstractions that illustrate the forces acting on a 
body, show no context; other diagrams show limited context; most photographs establish considerable context as a result of their medium- to long-shot range. A final placement on the context scale is difficult to establish here since both ends of the scale are represented and the high number of visuals, with their varying salience, make counting impractical. At the same time, because each end is represented and because the high number of visuals allows for the realization of most variables (for example, salient and backgrounded diagrams with context and without), a final placement of medium seems appropriate. Visual elements in this text are represented both as abstractions and as fully detailed representations. The most common abstractions are the free-body diagrams while the photographs and other drawings illustrating real-world problems show full representational detail. Again, the difficulty here is in determining this criterion's scalar placement. As with the context criterion, a medium placing seems most appropriate, for the same reasons as mentioned above. Since many of the drawings, however, do show some level of representational detail, this measurement is placed slightly higher.

Summary. The coding orientations of the texts differ: the Oxford and Garnet texts show a technological orientation while Hibbeler's is naturalistic. A graph reporting the visual scale results summarizes this analysis in Figure 3. One of the most significant consequences of this difference relates to specificity. Technological orientations, with their low rankings on each of the visual scales, feature visual participants that tend to be generically generalized. Naturalistic codings call attention to more specified visual participants, portraying the precise qualities and characteristics associated with the participant. For example, the naturalistic coding of photographs, and the free-body diagrams associated with the photographs, works to illustrate the discrete forces acting on a body in the particular portrayed environment. In contrast, the Oxford text 
shows highly abstracted diagrams of a ship, a spring balance, and a slope that are each intended to illustrate a force as a generic experience associated with a generic setting. Although all three texts also demonstrate limited evidence of abstract orientations, in their use of diagrams and graphs, this abstract coding is not the primary orientation. It should be noted, too, that the six Oxford diagrams are extremely abstracted, almost naive in their representation, and the result of this perceived naivete is reduced credibility, especially in context of the otherwise technological orientation

This analysis also reveals interesting indications of disciplinary self-identification and authority. Hibbeler encodes itself naturalistically and thus establishes an identity and authority based on the natural world. The EAP textbooks encode engineering technologically, establishing their identity and authority in the world of technology. This suggests that the EAP textbooks, or textbook writers, see engineering as a technical interpretation of reality, a very different interpretation than the naturalistic encoding established in the engineering textbook itself.

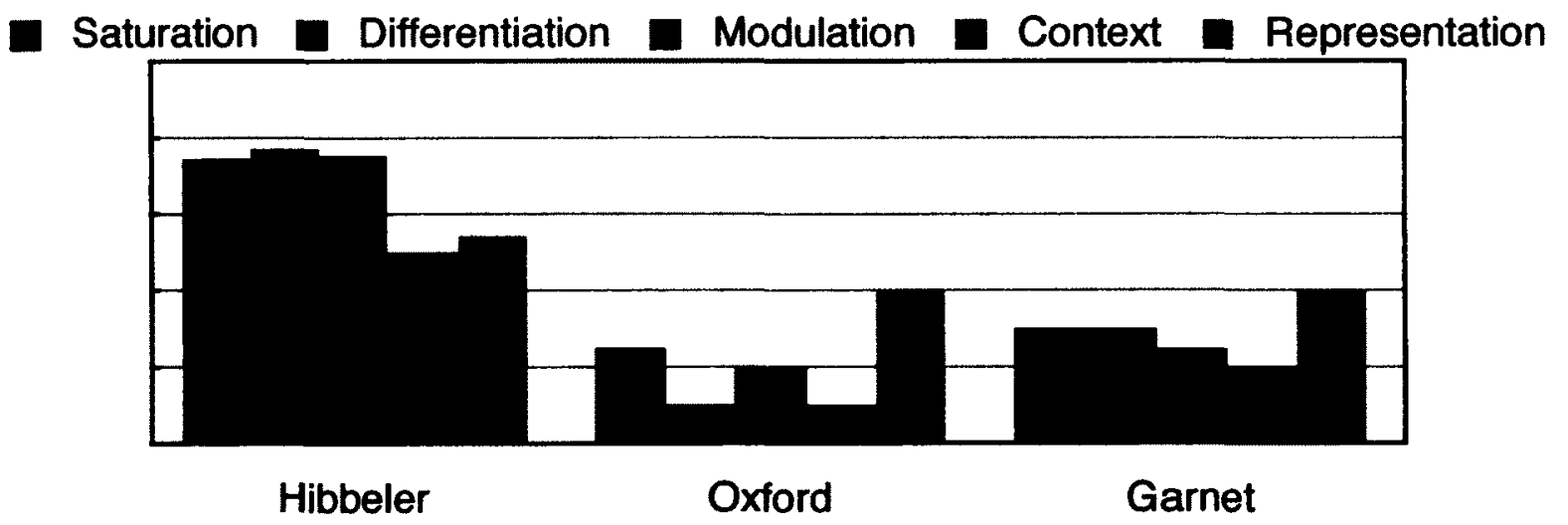

Figure 3: Coding orientation summary showing visual scales by text 


\section{Intersemiotic Analysis}

The intersemiotic analysis that I employed is the intermodal identification system set out by Unsworth and Cléirigh (2009). This system is based on the concept of relational grammatical identification and sees language and images as identifying one another in multimodal ensembles. When language identifies an image, the language is said to gloss the visual. Images identify language elements or language configurations. When identifying elements, three types of identification are possible: intensive, possessive, and circumstantial. When identifying configurations, the three types of identification are manner, cause: result or consequence, and cause: reason or condition.

Oxford. The 'spring balance' multimodal ensemble consists of one written paragraph and one accompanying diagram, appearing immediately below the written paragraph. The analysis reveals that intermodal identification is accomplished equally through the visualization of language elements and language configurations, as summarized in Table 15, below. The analysis shows two instances of possessive identification in which the visual identifies additional parts of a verbalized element. For example, the verbal element spring balance in the clause the spring balance can be calibrated in newtons is shown in the accompanying diagram with calibration marks. The two instances of images visualizing language configurations include one example in which a causal result is visualized and one example in which manner is visualized. In the causal result example, the verbalized configuration is the lengthy The weight on the balance pulls the spring down. To give equilibrium, the spring pulls up to oppose that weight. The diagram shows a spring balance with a weight attached to a hook on the bottom of the spring. The coils of the spring are not stretched out nor are they compressed; hence, they must be in equilibrium. The 
visual, then, is showing the result of equilibrium as a state in which the spring's coils are neither stretched out nor compressed. The difficulty with this example of intermodal identification, however, is a lack of indication to the reader as to which part of the verbal configuration is being visualized. Until the reader realizes that the diagram is visualizing such a lengthy piece of language (two sentences or four clauses), the reader may conclude that the diagram is inaccurate in showing the spring as not stretched out (as I did). The analysis also reveals two instances of glossing, in which language identifies an image. In both instances, an accompanying mathematical term also appears in the verbal text and in the image. For example, the verbalized upward force of the text identifies the image's upward arrow; the accompanying mathematical term, $F I$, appears immediately after the verbal element and as part of the visual diagram.

Garnet. The 'friction' multimodal ensemble consists of a unified cluster of three photographs and the opening paragraph of the 'friction' reading. Although the photographs and the reading are on facing pages, and not visually presented as a unified multimodal ensemble, the instructional text directs students to look at the photographs, read parts of the reading, and draw connections between the two. The analysis, presented in Appendix $\mathrm{H}$ and summarized in Table 15 below, shows that this ensemble uses intermodal identification to visualize language elements only. Language configurations are not visualized in this text. Of the five visualized language elements, two are intensive, two are possessive, and one is circumstantial. The text's opening clause When two objects are in contact shows all three types of identification with the accompanying photographs of various metal objects. For example, the photographs show the shape and texture of the verbalized objects, thus providing an intensive identification. A possessive identification is created with the photographs showing additional parts of the verbalized objects, such as 
the two interlocking rings that comprise one of the objects in the photograph. A circumstantial identification is also constructed with the photographs visualizing the location of the verbalized object's parts, such as the location of one of the interlocking rings as touching the inside of the other ring. The analysis also reveals that this text does not verbally gloss the images.

Hibbeler: Forces. In the 'springs' multimodal ensemble, one written paragraph with a diagram of three springs immediately below, the analysis shows that intermodal identification is achieved almost equally through visualization of language elements and configurations, as summarized in Table 15, below. The text employs five visualizations of language elements and seven of language configurations. Of the language element visualizations, three are intensive and thus visualize qualities of verbalized elements, and two are circumstantial, visualizing locations of verbal elements. All three intensive visualizations show the shape of the verbal element. For example, in the clause measured from its unloaded position (where its refers to the spring), the diagram reflects the verbalized unloaded position of the spring in the shape of its coils as neither stretched out nor compressed. In a similar example, in the clause the spring shown in Fig 3-1 has an undeformed length $l o=0.4 \mathrm{~m}$, the same diagram visualizes the verbalized undeformed length as coils that are neither stretched out nor compressed. In these two examples, intermodal identification constructs a semantic relationship of equality between unloaded position and undeformed length, since both verbalizations refer to the same coils of the diagram.

Of the seven visualized language configurations, six show causal results and one shows a causal reason. In the example of the verbal configuration If $s$ is positive (where $s$ refers to the distance that the spring is stretched out or that it is compressed), the diagram shows two different springs, one with $s$ as undeformed (that is, not stretched out or compressed) and the other with $s$ 
as deformed (stretched out in this case, since $s$ is positive). The image, then, visualizes the spatial difference of the distance between the undeformed spring's length and the stretched out, deformed length. This distance is also represented mathematically in the visual as $s=0.2 \mathrm{~m}$. The text's following clause, $F$ "pulls" on the string, is associated with the diagram's elongated spring, and the image again visualizes causal results of the language. In this case, the spring is shown with elongated coils, and as longer than the other two springs in the diagram. The analysis also reveals that this text shows no instances of verbal glosses.

Hibbeler: Friction. The 'friction' multimodal ensemble consists of one written paragraph and the three diagrams surrounding it. The analysis, presented in Appendix $\mathrm{H}$ and summarized in Table 15 below, reveals that this ensemble uses intermodal identification of visualized language elements and configurations, but favours visualization of configurations. Two language elements are visualized, both intensive identifications showing the texture of a surface. In one example, the language element rough horizontal surface is visualized in one of the diagram's as a pointillistic floor. Seven language configurations are visualized: three show manner, two show results, and two more show reason. In the verbal configuration pulling horizontally on a block of uniform weight, the visual shows the non-verbalized manner of pulling: an arrow emanates from the centre portion of the block's side, not from the top portion nor from the bottom portion of the block's side. In the verbal configuration For equilibrium, the normal forces must act upward, to balance the block's weight $W$, the diagram shows the causal result of the language: the box is sitting evenly, not tipping over or falling because upward arrows, representing the normal forces, and the downward arrow, representing the block's weight, are in balance. In the causal reason example, the diagram shows the reason for the verbal configuration each reactive force contrib- 
utes to both a frictional component $\Delta F n$ and a normal component $\Delta N n$. Reactive forces are able to contribute to frictional and normal components because the reactive forces develop in the exact same place - the protuberant points of contact between the surfaces - as the frictional and normal forces. This analysis also shows two examples of language gloss. The verbal uniform weight glosses the mathematical $W$ in the diagram.

Summary. A summary of intermodal relations is presented in Table 15 and shows relations by type and sub-type. The summary shows different patterns of intermodal identification in the EAP texts and in Hibbeler. Oxford visualizes language elements and configurations equally while Garnet visualizes only language elements. Both of these patterns are in contrast to Hibbeler which visualizes language configurations either more frequently, as in the case of the Hibbeler: Forces multimodal ensemble, or much more frequently, as with the Hibbeler: Friction ensemble. The Hibbeler: Forces ensemble relies strongly on causal result visualizations, showing six occurrences, while the Hibbeler: Friction ensemble uses all three sub-types of visual configuration. In contrast, Oxford visualizes one causal result and one manner. (The Garnet ensemble shows no instances of visualized configurations.) This pattern seems to show a far greater concern in the Hibbeler ensembles for causes and explanations. The visualization of language configurations is the more complex type of intermodal identification since it multiplies meaning, which suggests that Hibbeler is using this type of intermodal identification, and its multiplied meanings, as a pedagogic tool to aid in the understanding of difficult concepts. 
Table 15

Intermodal identification summary, by text

\begin{tabular}{|l|l|l|l|l|l|}
\hline \multicolumn{2}{|c|}{ Intermodal Feature } & Oxford & Garnet & \multicolumn{1}{c|}{$\begin{array}{c}\text { Hibbeler: } \\
\text { Forces }\end{array}$} & \multicolumn{1}{c|}{$\begin{array}{c}\text { Hibbeler: } \\
\text { Friction }\end{array}$} \\
\hline $\begin{array}{l}\text { Number of visual- } \\
\text { ized language ele- } \\
\text { ments, by type }\end{array}$ & intensive & - & 2 & 3 & 2 \\
\cline { 2 - 7 } & possessive & 2 & 2 & & \\
\cline { 2 - 7 } & circumstantial & - & 1 & 2 & 2 \\
\hline Total elements & 2 & 5 & 5 & 3 \\
\hline \multirow{2}{*}{$\begin{array}{l}\text { Number of visual- } \\
\text { ized language con- } \\
\text { figurations, by type }\end{array}$} & manner & 1 & - & & 2 \\
\cline { 2 - 7 } & causal: result & 1 & - & 6 & 2 \\
\hline & causal: reason & - & - & 1 & 7 \\
\hline Total configurations & & 2 & 0 & 7 & 2 \\
\hline Number of language glosses & 2 & - & - & \\
\hline
\end{tabular}

Concerning the visualization of verbal elements, again different patterns emerge among the texts. Oxford uses only possessive identification while Garnet uses all three types, almost equally. In contrast, the Hibbeler texts both prefer intensive identification. Intensive identifications visualize qualities of participants, showing that Hibbeler, again, is concerned with fuller specifications of its objects of study.

The Oxford ensemble and both Hibbeler ensembles share one pattern in which mathematical symbols or expressions are introduced in the text and then their location visualized in the accompanying diagrams. More specifically, all ensembles show a mathematical element in the written text and the same mathematical element in the visual diagram. For example, the symbol $W$, for weight, appears in the Oxford text and in its accompanying diagram. The placement of the mathematical item in the visual is important, however: each mathematical item is put in its most correspondingly concrete place in the diagram. Thus, $W$ is placed next to the block in the dia- 
gram. A Hibbeler example takes the mathematical $\Delta N n$, or normal forces, from the text and places the term in the diagram along the floor in which those forces are distributed, thus demonstrating the location of the mathematical concept. Unsworth and Cleirigh's (2009) system of intersemiotic relations allows us to see this placement of the mathematical term in the diagram not as simple repetition of the textual term but as a mathematical visualization of location of the mathematical concept.

One further point about the multimodal ensembles. In the Hibbeler ensembles, the visual elements are either placed directly below the written elements or are placed around the written ensembles such that, during reading, the reading path remains horizontal in its movement to the visual. In other words, if a line of text refers to the visual, the visual is to the immediate horizontal left or right, in the page margins. The eye is not required to search for the visual. The Oxford text integrates its visuals underneath their corresponding paragraphs but the Garnet ensemble, while showing the written and visual elements on facing pages, does not visually integrate the two modes. 


\section{Chapter Five \\ Discussion and Implications}

The purpose of this study was to compare verbal, visual, and intersemiotic representations of subject matter in EAP textbooks and an undergraduate engineering textbook in order to determine whether EAP publishers' claims that their textbooks help students access undergraduate, discipline-specific textbooks are realistic or not. The findings show significant differences in the representations constructed by the two types of textbooks, suggesting that publishers' claims are not realistic.

\section{Discussion}

The undergraduate Hibbeler Mechanical Engineering textbook represents its subject matter from a naturalistic standpoint; that is, Hibbeler presents its content as the phenomena, actions, and events of the natural world. In contrast, both EAP textbooks represent the world of engineering from a technical perspective that positions engineering subject content as the specialized information of a technological world. Interestingly, however, from an applied linguistics standpoint, the Hibbeler text is revealed as the technical textbook and the EAP textbooks as concerned with the everyday world. The technical features of the Hibbeler text include its consistent choice of material processes to encode technical actions and its considerably smaller focus on attributive processes that describe and specify. The EAP texts, on the other hand, choose material processes and identifying processes of definition, both characteristic of everyday language (Eggins, 2004). From a language teaching and learning perspective, one of the practical problems with representing subject matter in terms of different process types (material versus the attributive or identifying processes) is that process types are constructed differently in the grammar, as an SFL analysis is designed to show. In choosing different process types to represent experience, not only is a 
different reality represented, but that representation is grammatically different than the grammar of other processes. For example, linguistic material processes and visual unidirectional processes construct Goals, or in more traditional grammar terms 'objects', while identifying processes do not. Therefore, the representations in the EAP texts analyzed in the present study introduce and accustom learners not to the grammar of the analyzed engineering textbook but, it would seem, to the grammar of EAP texts.

Further evidence of Hibbeler's technical focus includes its use of nominalization in descriptions of phenomena and its development of class/sub-class taxonomies and part/whole meronymies (Eggins, 2004, Halliday, 1985/2004). The Hibbeler text also constructs complex technical collocations and signals non-technical lexical terms with distancing quotes. In contrast, the EAP texts tend not to represent subject matter as nominalizations, do not develop taxonomies, and show limited use of technical vocabulary. Linguistically and visually, the two types of texts also construct their topics and textual participants differently, again showing differences along technical/everyday dimensions. In the Hibbeler text, topics are specified with very focused areas of attention, and participants are highly characterized, verbally and mathematically, in ways specific to the discipline. In the EAP texts, topics are generalized, and participants are generic, both features of common language and everyday life (Eggins, 2004).

One of the more striking differences between the EAP texts and the Hibbeler text is how each deals with mathematics. In Hibbeler, mathematics plays a significant role. Mathematical elements are frequently and regularly constructed as textual participants in written text and in visuals, in simple and complex representations of phenomena and actions. The EAP textbooks have almost excised mathematics from their content, leaving only a few simple mathematical 
symbols and even fewer complex terms. Without a doubt, one of the reasons for this excision is because EAP books are 'taught' by language teachers, teachers who are probably untrained in mathematics, and, the assumption goes, either afraid of mathematics or too unknowledgeable to manage mathematics.

Yet multimodal theory highlights that meaning is a construction of all the modes that are present in a communication; it is not possible to remove, or almost remove, a mode and maintain the same meanings. Therefore, the removal of mathematics in the EAP texts dramatically affects how the texts mean. This concept is most evident in the intersemiotic analysis of multimodal ensembles in which new meanings are generated in the interaction of modes. In the Hibbeler text, mathematics plays an integral part in generating these new meanings. Mathematics' removal, of course, equals the removal of the intersemiotic meanings it helps develop.

The multimodal ensembles that the texts create are also different in other important ways. In the Hibbeler ensembles, the verbal and visual modes are highly integrated, even to the line of text in which the reference from one to the other occurs. Multimodal findings in the undergraduate textbook confirm Royce's $(2002,2007)$ focus on the significance of helping second language learners develop a multimodal communicative competence. In the Garnet EAP text, however, ensembles are not visually or spatially integrated; the verbal and visual modes of the ensemble are connected by instructional text that asks learners to refer from one to the other. The Oxford text shows integrated ensembles, although ensembles occasionally overflow the page: the written text occurs at the bottom of the left-hand page, while the visual with which its semantically integrated overflows to the top of the right-hand page. While neither of the multimodal layouts of the Garnet and Oxford texts would necessarily preclude the multiplied meanings available through 
intersemiosis, the analysis shows that these meanings were either limited, as with Oxford, or not produced at all, as with Garnet. These results, then, seem to validate Mayer's (2008) and Tufte's (2006) visual design principles that call for the complete integration of all modes in order to enhance learning and "assist understanding of the content" (Tufte, 2006, p. 88, italics in original) in that gaps in modal integration fail to capture opportunities for learning and understanding. As pedagogic resources helpful in the explication of difficult concepts, multimodal ensembles are under-used in the EAP texts. As well as being under-used, and therefore not reflective of how multimodal ensembles are used in the engineering text, the shortcomings of the EAP ensembles do not permit for explorations in how ensembles construct meanings in English.

The presence of visuals and multimodal ensembles affects the way a textbook page looks, a significant point when taken in consideration with Olive and Passerault's (2012) visuospatial representations. While this present study did not explicitly examine layout or page design, the integration of modes and the placement of visuals in Hibbeler points to highly specific uses of page space. For example, in Hibbeler, some diagrams are placed immediately next to photographs so that the visual content of the photograph can be mirrored in the diagram and thus represented diagrammatically. In this and other ways, Hibbeler's pages produce visuospatial representations that are highly distinctive. In contrast, the visuospatial representations developed by the EAP texts, neither of which place visuals or other information in this manner, are not reflective of patterns developed in the engineering textbook. This point is significant since it is theorized that visuospatial representations help learners find and retrieve information in a text, skills that are particularly relevant for users of textbooks. 


\section{Implications}

A number of important implications exist for EAP materials design with respect to Mechanical Engineering. At the most general level, rather than representing EAP's vision of Mechanical Engineering, EAP textbooks need to accurately represent how Mechanical Engineering represents itself. This study shows that the Hibbeler text uses what Applied Linguistics refers to as technical language (but is recognized by engineers as language that expresses the natural world), in both grammatical and lexical realizations. The subject matter of Mechanical Engineering needs to be represented in technical language using process types as found in originating texts, thus ensuring a similarity in representation semantically and grammatically. Highly specified topics and participants need to be constructed and specified through taxonomies and meronymies, complex and detailed collocations of technical terms, and the frequent use of nominalized entities. In order to help explicate complex concepts, mathematical symbols, terms, and expressions must be included and integrated into written text and visual elements. Although from a language perspective, English teachers may not know how mathematical terms clarify meaning, in Mechanical Engineering, they help to do just that. Multimodal ensembles that fully integrate verbal and visual modes must also be employed to aid in comprehension, especially in their capacity to develop meaning potential and multiply meanings through the process of intersemiosis. Finally, visuospatial representations need to be recognized; Hibbeler constructs distinctive patterns of representation and EAP textbooks should, at the very least, construct similar representations to acclimatize students to the discipline.

Each of the above points might act as yardsticks in helping to determine how much content is necessary in EAP textbooks for Mechanical Engineering students and in other subject con- 
tent texts. As noted earlier, this problem is a central issue in EAP today (Belcher, 2006). My study suggests that target-discipline content in EAP textbooks needs to be sufficient enough to address a range of important discipline-specific features. These features, as indicated by the present mechanical engineering study, include overall representations of the target discipline, levels of technicality as expressed grammatically and lexically in the construction of topics and participants, multimodal ensembles and their integration of modal resources, and the inclusion of simple and complex mathematical participants in written text and visual elements. Almost certainly the reason mathematics has been nearly excluded from the analyzed EAP textbooks is more to do with accommodating language teachers rather than accommodating the target discipline. However, this study suggests that mathematics cannot be eliminated from an EAP textbook without damage to meaning. What needs to be eliminated is the perception that language teachers are incapable of working with mathematics. Shaw's (2006) assertion that discourse analysts need to understand how mathematics and language work together, rather than investigating the mathematics itself, needs to be extended to include language teachers.

This point draws attention to the content that English language teachers need to teach and thus brings me back to the issues raised in my discussion of Content-Based Instruction. Since Engineering is a discipline informed by visual and mathematical principles, EAP must take its lead from its originating discipline and represent a multimodal conception of how Engineering English means. This means embracing the visuals and mathematics that help constitute Engineering, as well as tending to the more traditionally linguistic concerns of verbal language. This inclusion may necessitate some reconceptualizations of what it means to teach English and be an 
English teacher, reconceptualizations that might be captured by thinking of ourselves, not so much as English language teachers, but as English meaning teachers. 


\section{References}

Ackerley, K., \& Coccetta, F. (2007). Enriching language learning through a multimedia corpus. $\operatorname{ReCALL,~19(3),351-370.~}$

Atkinson, R. K. (2005). Multimedia learning of mathematics. In R. Mayer (Ed.), The Cambridge Handbook of Multimedia Learning (pp. 393 - 408). Cambridge: Cambridge University Press.

Belcher, D. (2006). English for specific purposes: Teaching to perceived needs. TESOL Quarterly, 40(1), 133-156.

Benesch, S. (2001). Critical English for academic purposes: Theory, politics, and practice. New Jersey: Lawrence Erlbaum.

Bezemer, J., \& Kress, G. (2008). Writing in multimodal texts: A social semiotic account of designs for learning. Written Communication, 25(2), 166-195.

Bezemer, J., \& Kress, G. (2009). Visualizing English: A social semiotic history of a school subject. Visual Communication, 8(3), 247-262.

Brown, V. (1993). Decanonizing discourses: Textual analysis and the history of economic thought. In W. Henderson, T. Dudley-Evans, \& R. Backhouse (Eds.), Economics and language (pp. 64-84). London: Routledge.

Chapelle, C. (2010). The spread of computer-assisted language learning. Language Teaching, $43(1), 66-74$.

Coffin, C., \& Donohue, J. P. (2012). English for academic purposes: Contributions from systemic functional linguistics and academic literacies. Journal of English for Academic Purposes, $1 I(1), 1-3$ 
Dun, M., Howey, D., Ilic, A., \& Regan, N. (2010). English for Mechanical Engineering in higher education studies. Reading: Garnet.

Dudley-Evans, T., \& St. John, M. (1998) Developments in English for specific purposes: multi-disciplinary approach. Cambridge: Cambridge University Press.

Eggins, S. (2004). An introduction to systemic functional linguistics (2nd ed.). London: Continuum.

Fairclough, N. (1989/2001). Language and power. Harlow: Pearson Education.

Fairclough, N. (1995/2010). Critical discourse analysis: The critical study of language. Harlow: Pearson Education.

Glendinning, E., \& Glendinning, N. (2001). Oxford English for Electrical and Mechanical Engineering. Oxford: Oxford University Press.

Halliday, M. A. K. (1985/2004). An introduction to functional grammar. London: Edward Arnold. Pittsburgh: University of Pittsburgh Press.

Halliday, M. A. K., \& Martin, J. R. (1993). Writing science: Literacy and discursive power. Pittsburgh: University of Pittsburgh Press.

Hammond, J. \& Macken-Horarik, M. (1999). Critical literacy: Challenges and questions for ESL classrooms. TESOL Quarterly, 33(3), 528-544.

Harwood, N. (2005). What do we want EAP teaching materials for? Journal of English for Academic Purposes, 4(2), 149-161.

Hibbeler, R. C. (2007). Engineering mechanics: Statics and dynamics (11 th ed.). Upper Saddle River: Pearson Prentice Hall. 
Hyland, K. (2006). English for academic purposes: An advanced resource book. London: Routledge.

Hyland, K. (2009). Academic discourse. London: Continuum.

Hyland, K., \& Hamp-Lyons, L. (2002). EAP: Issues and directions. Journal of English for Academic Purposes, 1(1), 1-12.

Hymes, D. (1972). On communicative competence. In J. B. Pride \& J. Holmes (Eds.), Sociolinguistics (pp. 269-293). Harmondsworth: Penguin Books.

Iedema, R. (2003). Multimodality, resemiotization: Extending the analysis of discourse as multisemiotic practice. Visual Communication, 2(1), 29-57.

Jewitt, C. (2008). Multimodality and literacy in school classrooms. Review of Research in Education, 32, 241-267.

Kress, G. (2000). Multimodality: Challenges to thinking about language. TESOL Quarterly, $34(2), 337-340$.

Kress, G. (2008) Meaning and learning in a world of instability and multiplicity. Studies in Philosophy and Education, 27(4), 253-266.

Kress, G. (2010). Multimodality: A social semiotic approach to contemporary communication. Routledge: London.

Kress, G. \& Burn, A. (2005). Pictures from a rocket: English and the semiotic text. English Teaching: Practice and Critique, 4(1), 95-105.

Kress, G., Charalampos, T., Jewitt, C., \& Ogborn, J. (2001). Multimodal teaching and learning: The rhetorics of the science classroom. London: Continuum. 
Kress, G., \& van Leeuwen, T. (2001). Multimodal discourse: The modes and media of contemporary communication. London: Arnold.

Kress, G., \& van Leeuwen, T. (1996/2006). Reading images: The grammar of visual design. London: Routledge.

Lemke, J. (1998). Multiplying meaning: Visual and verbal semiotics in scientific text. In J. R. Martin \& R. Veel (Eds.). Reading science: Critical and functional perspectives on discourses of science (pp. 87-113). London: Routledge.

Lemke, J. (2002). Multimedia semiotics: Genres for science education and scientific literacy. In M. Schleppegrell \& M. Cecilia Colombi (Eds.). Developing advanced literacy in first and second languages (pp. 21-44). New Jersey: Lawrence Erlbaum.

Lyster, R., \& Ballinger, S. (2011). Content-based language teaching: Convergent concerns across divergent contexts. Language Teaching Research, 15(3), 279-288.

Martinec, R., \& Salway, A. (2005). A system for image-text relations in new (and old) media. Visual Communication, 4(3), 337-371.

Mayer, R. (2005). Cognitive theory of multimedia learning. In R. Mayer (Ed.), The Cambridge handbook of multimedia learning (pp. 31-48).

Mayer, R. (2008). Multimedia literacy. In J. Coiro, M. Knobel, C. Lankshear, \& D. J. Leu (Eds.), Handbook of research on new literacies (pp. 359-376.

McDonough, J. (2010). English for specific purposes: A survey review of current materials. ELT Journal, 64(4), 467-477.

New London Group. (1996). A pedagogy of multiliteracies: Designing social futures. Harvard Educational Review, 66(1), 60-92. 
Olive, T., \& Passerault, J. (2012). The visuospatial dimension of writing. Written Communication, 29(3), 326-344.

O'Toole, M. (1994). The language of displayed art. London: Leicester University Press.

Richards, J. (2001). Curriculum development in language education. Cambridge: Cambridge University Press.

Richardson, P. (2004). Reading and writing from textbooks in higher education: A case study from Economics. Studies in Higher Education, 29(4), 505-521

Roth, W., Pozzer-Ardenghi, L., \& Han, J. Y. (2005). Critical graphicacy: Understanding visual representation practices in school science. Dordrecht: Springer.

Royce, T. (2002). Multimodality in the TESOL classroom: Exploring visual-verbal synergy. TESOL Quarterly, 36(2), 191-205.

Royce, T. (2007). Intersemiotic complementarity: A framework for multimodal discourse analysis. In T. Royce \& W. Bowcher (Eds.). New directions in the analysis of multimodal discourse. (pp. 63-109). New Jersey: Lawrence Erlbaum.

Royce, T. (2007). Multimodal communicative competence in second language contexts. In T. Royce \& W. Bowcher (Eds.). New directions in the analysis of multimodal discourse. (pp. 361-390). New Jersey: Lawrence Erlbaum.

Saint-Martin, F. (1994). Verbal and visual semantics. In T. Sebeok \& J. Umiker-Sebeok (Eds.), Advances in visual semiotics: The semiotics web 1992-93. Berlin: Mouton de Gruyter. Shaw, P. (2006). Relations between text and mathematics across disciplines. In K. Hyland \& M. Bondi (Eds.). Academic discourse across disciplines (pp. 103-122). Bern: Peter Lang. Swales, J. (1980). ESP: The textbook problem. The ESP Journal, 1(1), 11-23. 
Swales, J. (1995). The role of the textbook in EAP writing research. English for Specific Purposes, 14, 3-18.

Tomlinson, B. (2012). Materials development for language learning and teaching. Language Teaching, 45(2), 143-179.

Tribble, C. (2009). Writing academic English-a survey review of current published resources. ELT Journal, 63(4), 400-417.

Tufte, E. (2006). Beautiful evidence. Cheshire: Graphics Press.

Unsworth, L. (2006). Towards a metalanguage for multiliteracies education: Describing the meaning-making resources of language-image interactions. English Teaching: Practice and Critique, 5(1), 55-76.

Unsworth, L. (2008). Multimodal semiotic analyses and education. In L. Unsworth (Ed.), Multimodal semiotics: Functional analysis in contexts of education (pp. 1-12). London: Continuum.

Unsworth, L., \& Cléirigh, C. (2009). Multimodality and reading: The construction of meaning through image-text interaction. In C. Jewitt (Ed.), The Routledge handbook of multimodal analysis (pp. 151-163). New York: Routledge.

van Leeuwen, T. (1999). Speech, music, sound. London: Macmillan.

van Leeuwen, T. (2005). Introducing social semiotics. London: Routledge.

Wakefield, J. F. (June, 1998). A brief history of textbooks: Where have we been all these years?

Paper presented at Text and Academic Authors Meeting, St. Petersburg, FL.

Wilson, J. (2010). Recent IELTS materials. ELT Journal, 64(2), 219-232. 


\section{Appendix A \\ Verbal Data from Oxford English for Electrical and Mechanical Engineering}

\section{Forces in engineering}

To solve the ship problem, we must look at the forces on the ship (Fig. 1). The weight, W, acts downwards. That is the gravity force. The buoyancy force, B, acts upwards. Since the ship is in equilibrium, the resultant force is zero, so the magnitudes of $B$ and $W$ must be the same.

Another very important force in engineering is the one caused by elasticity. A good example of this is a spring. Springs exert more force the more they are stretched. This property provides a way of measuring force. A spring balance can be calibrated in newtons, the unit of force. The block in Fig. 2 has a weight of 10 newtons. The weight on the balance pulls the spring down. To give equilibrium, the spring pulls up to oppose that weight. This upward force, F1, equals the weight of the block, W.

It is important to get the distinction between mass and weight absolutely clear. Mass is the quantity of matter in an object. Weight is the force on that object due to gravity. Mass is measured in kilograms, whereas weight, being a force, is measured in newtons.

We have looked at buoyancy, elasticity, and gravity. There is a fourth force important in engineering, and that is friction. Friction is a help in some circumstances but a hindrance in others. Let us examine the forces on the box (Fig. 3). Firstly, there is its weight, W, the gravity force, then there is the reaction, $R$, normal to the plane. $R$ and $W$ have a resultant force trying to pull the box down the slope. It is the friction force, F, acting up the slope, that stops it sliding down. (all 4 paragraphs from p. 32) 


\section{Appendix B \\ Verbal Data from Garnet's English for Mechanical Engineering in Higher Education}

\section{Coefficient of Friction}

The coefficient of friction, $\mu$, is a scalar value. It is the ratio between the normal force, $\mathrm{N}$, of one object on another and the force of resistance, $R$. This ratio differs according to the materials in contact. A rubber tyre on a road has a high coefficient, whereas a piston inside a cylinder has a low one. There are two basic kinds of friction.

\section{Static Friction: $\mu s$}

Static friction is what makes stationary objects difficult to move. The direction of this force is along the contact surface and it is equal and opposite to the applied force. The magnitude of the static friction force is given by $f \mathrm{~s}=\mu \mathrm{sN}$, where $\mu$ is the coefficient of friction and $\mathrm{N}$ is the normal force. (both preceding paragraphs from p. 46)

\section{Friction: blessing and curse}

When two objects are in contact and one object is moved past the other, a force is created which resists the movement of the objects: it is called friction. Friction occurs in all machines and is usually considered detrimental to the machine's efficiency, but there are times when it is required for the correct functioning of a machine. Although the effect that friction has on an engineering system depends on the nature of the materials that are in contact, certain facts are known to be consistent for all materials.

In many mechanical design specifications, engineers must bear in mind several principles of friction. These are, specifically: Friction is a force that opposes movement between two objects. There is always some friction when two touching surfaces move relative to one another. To initiate movement from a standstill, more force is needed to overcome friction than the force required to maintain a constant speed when the objects are already in motion. Generally speaking, the smoother the surfaces, the lower the friction. Friction can be reduced by lubrication and by good design. (both preceding paragraphs from p. 49) 


\section{Appendix C \\ Verbal Data for Hibbeler: Forces from Engineering Mechanics: Statics \& Dynamics}

Mass. Mass is a property of matter by which we can compare the action of one body with that of another. This property manifests itself as a gravitational attraction between two bodies and provides a quantitative measure of the resistance of matter to a change in velocity. (p. 4)

Force. In general, force is considered as a "push" or "pull" exerted by one body on another. This interaction can occur when there is direct contact between the bodies, such as a person pushing on a wall, or it can occur through a distance when the bodies are physically separated. Examples of the latter type include gravitational, electrical, and magnetic forces. In any case, a force is completely characterized by its magnitude, direction, and point of application. (p. 4)

Springs. If a linearly elastic spring is used for support, the length of the spring will change in direct proportion to the force acting on it. A characteristic that defines the "elasticity" of a spring is the spring constant or stiffness $\mathbf{k}$. The magnitude of force exerted on a linearly elastic spring which has a stiffness $k$ and is deformed (elongated or compressed) a distance $s$, measured from its unloaded position, is $\mathrm{F}=\mathrm{ks}$. Here $\mathrm{s}$ is determined from the difference in the spring's deformed length and its undeformed length lo, i.e., $s=1$ - lo. If $s$ is positive, $F$ "pulls" on the spring; whereas if $\mathrm{s}$ is negative, F must "push" on it. For example, the spring shown in Fig 3-1 has an undeformed length $l o=0.4 \mathrm{~m}$ and stiffness $\mathrm{k}=500 \mathrm{~N} / \mathrm{m}$. To stretch it so that $1=0.6 \mathrm{~m}$, a force $\mathrm{F}=$ $\mathrm{ks}=(500 \mathrm{~N} / \mathrm{m})(0.6 \mathrm{~m}-0.4)=100 \mathrm{~N}$ is needed. (both "Springs" paragraphs from p. 84) 


\section{Appendix D \\ Verbal Data for Hibbeler: Friction from Engineering Mechanics: Statics \& Dynamics}

\section{Characteristics of Dry Friction}

Friction can be defined as a force of resistance acting on a body that prevents or retards slipping of the body relative to a second body or surface with which it is in contact. This force always acts tangent to the surface at points of contact with other bodies and is directed so as to oppose the possible or existing motion of the body relative to these points. (p. 393)

Theory of Dry Friction. The theory of dry friction is best explained by considering the effects caused by pulling horizontally on a block of uniform weight $W$ which is resting on a rough horizontal surface, Fig. 8-1a. To properly develop a full understanding of the nature of friction, it is necessary to consider the surfaces of contact to be nonrigid or deformable. The upper portion of the block, however, can be considered rigid. As shown on the free-body diagram of the block, Fig. 8-1b, the floor exerts a distribution of both normal force $\Delta \mathrm{Nn}$ and frictional force $\Delta \mathrm{Fn}$ along the contacting surface. For equilibrium, the normal forces must act upward to balance the block's weight $\mathrm{W}$, and the frictional forces act to the left to prevent the applied force $\mathbf{P}$ from moving the block to the right. Close examination of the contacting surfaces between the floor and block reveals how these frictional and normal forces develop, Fig. 8-1c. It can be seen that many microscopic irregularities exist between the two surfaces and, as a result, reactive forces $\Delta R n$ are developed at each of the protuberances. These forces act at all points of contact, and, as shown, each reactive forces contributes both a frictional component $\Delta \mathrm{Fn}$ and a normal component $\Delta \mathrm{Nn}$. (p. 394)

Impending Motion...Experimentally, it has been determined that the limiting static frictional force $F s$ is directly proportional to the resultant normal force $N$. This may be expressed mathematically as $F s=\mu s N$ where the constant of proportionality, $\mu s$ (mu "sub" s), is called the coefficient of static friction. Thus, when the block is on the verge of sliding, the normal force $\mathbf{N}$ and frictional force Fs combine to create a resultant Rs, Fig. 8-1e. The angle Øs that Rs makes with $\mathbf{N}$ is called the angle of static friction. (p. 395) 


\section{Appendix E \\ Selected Verbal Analyses of Transitivity}

Transitivity analysis: Oxford, by clause, paragraph 1

\begin{tabular}{|l|l|l|l|l|}
\hline 1 & & & To solve & the ship problem \\
\hline & & & Mental:cognition & Phenomenon \\
\hline
\end{tabular}

\begin{tabular}{|l|l|l|l|l|l|}
\hline 2 & & we & must look & at the forces & on the ship (Fig. 1). \\
\hline & & Sensor & Mental:perception & Phenomenon & Circ:location \\
\hline
\end{tabular}

\begin{tabular}{|l|l|l|l|l|}
\hline 3 & & The weight, $W$, & acts & downwards. \\
\hline & & Actor & Material & Circ:manner \\
\hline
\end{tabular}

\begin{tabular}{|l|l|l|l|l|}
\hline 4 & & That & is & the gravity force. \\
\hline & & Token & Identifying & Value \\
\hline
\end{tabular}

\begin{tabular}{|l|l|l|l|l|}
\hline 5 & & The buoyancy force, $B$, & acts & upwards. \\
\hline & & Actor & Material & Circ:manner \\
\hline
\end{tabular}

\begin{tabular}{|l|l|l|l|l|}
\hline 6 & Since & the ship & is & in equilibrium, \\
\hline & & Carrier & Attributive & Attribute \\
\hline
\end{tabular}

\begin{tabular}{|l|l|l|l|l|}
\hline 7 & & the resultant force & is & zero, \\
\hline & & Carrier & Attributive & Attribute \\
\hline
\end{tabular}

\begin{tabular}{|l|l|l|l|l|}
\hline 8 & so & $\begin{array}{l}\text { the magnitudes of } \\
\text { B and } W\end{array}$ & must be & the same. \\
\hline & & Carrier & Attributive & Attribute \\
\hline
\end{tabular}


Transitivity analysis: Hibbeler: Forces, by clause, paragraph 3

\begin{tabular}{|l|l|l|l|l|}
\hline 1 & If & a linearly elastic spring & is used & for support, \\
\hline $\mathbf{P}$ & & Goal & Material & Circ:cause \\
\hline
\end{tabular}

\begin{tabular}{|l|l|l|l|l|}
\hline 2 & & the length of the spring & will change & $\begin{array}{l}\text { in direct proportion to the force [acting } \\
\text { on it]. }\end{array}$ \\
\hline & & Actor & Material & Circ:manner \\
\hline
\end{tabular}

\begin{tabular}{|l|l|l|l|}
\hline 3 & $\begin{array}{l}\text { A characteristic [that defines the } \\
\text { "elasticity"] of a spring }\end{array}$ & is & the spring constant or stiffness $k$ \\
\hline & Carrier & Attributive & Attribute \\
\hline
\end{tabular}

\begin{tabular}{|l|l|l|}
\hline$\ldots$ & The magnitude of force [exerted on a linearly elastic spring]... & \\
\hline & & \\
\hline
\end{tabular}

\begin{tabular}{|l|l|l|l|l|}
\hline 4 & & which & has & a stiffness $k$ \\
\hline & & & Attribute:possess & Attribute \\
\hline
\end{tabular}

\begin{tabular}{|l|l|l|l|l|}
\hline 5 & and & $\begin{array}{l}\text { is deformed } \\
\text { (elongated or } \\
\text { compressed })\end{array}$ & a distances, \\
\hline $\mathbf{P}$ & & & Material & Range \\
\hline
\end{tabular}

\begin{tabular}{|l|l|l|l|l|}
\hline 6 & & & measured & from its unloaded position, \\
\hline & & & Material & Circ:manner \\
\hline
\end{tabular}

\begin{tabular}{|l|l|l|l|}
\hline 7 & $\begin{array}{l}\text { The magnitude of force [exerted } \\
\text { on a linearly elastic spring] } \ldots\end{array}$ & $\ldots$ is & {$[F=k s]$.} \\
\hline & Value & Identifying & Token \\
\hline
\end{tabular}




\begin{tabular}{|l|l|l|l|l|}
\hline 8 & Here & $s$ & is determined & $\begin{array}{l}\text { from the difference in the spring s deformed } \\
\text { length l and its undeformed length lo, }\end{array}$ \\
\hline $\mathbf{P}$ & & Phenomenon & Mental:cog & Circ:manner \\
\hline
\end{tabular}

\begin{tabular}{|l|l|l|l|l|}
\hline 9 & i.e., & & {$[s=l-l o]$.} & \\
\hline & & & Math process & \\
\hline
\end{tabular}

\begin{tabular}{|l|l|l|l|l|}
\hline 10 & If & $s$ & is & positive, \\
\hline & & Carrier & Attributive & Attribute \\
\hline
\end{tabular}

\begin{tabular}{|l|l|l|l|l|}
\hline 11 & & F & “pulls" & on the spring; \\
\hline & & Actor & Material & Circ:location \\
\hline
\end{tabular}

\begin{tabular}{|l|l|l|l|l|}
\hline 12 & whereas if & $s$ & is & negative, \\
\hline & & Carrier & Attributive & Attribute \\
\hline
\end{tabular}

\begin{tabular}{|l|l|l|l|l|}
\hline 13 & & $F$ & must "push" & on it. \\
\hline & & Actor & Material & Cir:location \\
\hline
\end{tabular}

\begin{tabular}{|l|l|l|l|l|}
\hline 14 & $\begin{array}{l}\text { For exam- } \\
\text { ple, }\end{array}$ & $\begin{array}{l}\text { the spring [shown } \\
\text { in Fig 3-1] }\end{array}$ & has & $\begin{array}{l}\text { an undeformed length }[l o=0.4 m] \text { and } \\
\text { stiffness }[k=500 \mathrm{~N} / \mathrm{m}] .\end{array}$ \\
\hline & & Carrier & Attributive & Attribute \\
\hline
\end{tabular}

\begin{tabular}{|l|l|l|l|l|}
\hline 15 & & & To stretch & it \\
\hline & & & Material & Goal \\
\hline
\end{tabular}




\begin{tabular}{|l|l|l|l|l|}
\hline 16 & so that & & {$[l=0.6 \mathrm{~m}]$,} & \\
\hline & & & Math process & \\
\hline
\end{tabular}

\begin{tabular}{|l|l|l|l|}
\hline 17 & $\begin{array}{l}\text { a force }[F=k s=(500 \mathrm{~N} / \mathrm{m})(0.6 \mathrm{~m} \\
-0.4)=100 \mathrm{~N}]\end{array}$ & is needed. & \\
\hline $\mathbf{P}$ & Phenomenon & Mental:affect & \\
\hline
\end{tabular}




\section{Appendix F}

\section{Selected Verbal Analyses of Reference}

Reference analysis: Oxford, paragraph 3

\begin{tabular}{|l|l|l|l|}
\hline Clause & $\begin{array}{c}\text { Presuming } \\
\text { Item }\end{array}$ & Type of Reference & \multicolumn{1}{|c|}{ Clause (participants bolded) } \\
\hline 1 & it & cataphoric & It is important \\
\hline 2 & the & cataphoric & to get the distinction between mass and weight absolutely clear. \\
\hline 3 & the / an & esphoric & Mass is the quantity of matter in an object. \\
\hline 4 & the / that & $\begin{array}{l}\text { cataphoric/ } \\
\text { anaphoric }\end{array}$ & Weight is the force on that object due to gravity. \\
\hline 5 & & & Mass is measured in kilograms, \\
\hline 6 & a & & whereas weight, being a force, is measured in newtons. \\
\hline
\end{tabular}

Reference analysis: Hibbeler: Forces, paragraph 1

\begin{tabular}{|l|l|l|l|}
\hline Clause & $\begin{array}{c}\text { Presuming } \\
\text { Item }\end{array}$ & Type of Reference & \multicolumn{1}{|c|}{ Clause (participants bolded) } \\
\hline 1 & a & & Mass is a property of matter \\
\hline 2 & $\begin{array}{l}\text { we, the, that, } \\
\text { another }\end{array}$ & $\begin{array}{l}\text { exophoric, espho- } \\
\text { ric, anaphoric x } 2\end{array}$ & $\begin{array}{l}\text { by which we can compare the action of one body with that of } \\
\text { nother. }\end{array}$ \\
\hline 3 & this, itself, a & $\begin{array}{l}\text { bridging, ana- } \\
\text { phoric x 2 }\end{array}$ & $\begin{array}{l}\text { This property manifests itself as a gravitational attraction } \\
\text { between two bodies }\end{array}$ \\
\hline 4 & a, the, a & esphoric & $\begin{array}{l}\text { and provides a quantitative measure of the resistance of mat- } \\
\text { ter to a change in velocity. }\end{array}$ \\
\hline
\end{tabular}


Appendix G

Selected Verbal Analyses of Lexical Relations

Lexical relations analysis: Garnet

\begin{tabular}{|c|c|c|c|c|}
\hline Taxonomic & Paragraph 1 & Paragraph 2 & Paragraph 3 & Paragraph 4 \\
\hline \multicolumn{5}{|l|}{ Classification } \\
\hline \multicolumn{5}{|l|}{ co-hyponymy } \\
\hline class/sub-class & $\begin{array}{l}\text { - scalar value: } \\
\text { - coefficient of } \\
\text { friction } \\
\text { - materials: } \\
\text { - rubber tyre, } \\
\text { road, piston, } \\
\text { cylinder }\end{array}$ & $\begin{array}{l}\text { - friction: } \\
\text { - static, kinetic }\end{array}$ & $\begin{array}{l}\text { - force: } \\
\text { - friction }\end{array}$ & $\begin{array}{l}\text { - force: } \\
\text { - friction }\end{array}$ \\
\hline contrast & - high:low & - move:stationary & & $\begin{array}{l}\text { - movement:standstill } \\
\text { - maintain:overcome }\end{array}$ \\
\hline synonymy & $\begin{array}{l}\text { coefficient of } \\
\text { friction:u }\end{array}$ & $\begin{array}{l}\text { - coefficient of } \\
\text { friction:u } \\
\text { - N:normal force }\end{array}$ & & - lower:reduced \\
\hline repetition & $\begin{array}{l}\text { - friction } \times 2 \\
\text { - force } \times 2\end{array}$ & $\begin{array}{l}\text { friction } \times 3 \\
\text { - force } \times 4\end{array}$ & $\begin{array}{l}\text { - object } \times 3 \\
\text { - friction } \times 3 \\
\text { - machine } \times 3 \\
\text { - materials } \times 2\end{array}$ & $\begin{array}{l}\text { - friction x } 6 \\
\text { - force } \times 3 \\
\text { - object x } 3\end{array}$ \\
\hline \multicolumn{5}{|l|}{ Composition } \\
\hline meronymy & $\begin{array}{l}\text { - coefficient of fric- } \\
\text { tion: } \\
\text { - normal force } \\
\mathrm{N}, \text { force of } \\
\text { resistance } \mathrm{R}\end{array}$ & $\begin{array}{l}\text { - static friction: } \\
\text { direction, magni- } \\
\text { tude (this compo- } \\
\text { sition isn't obvi- } \\
\text { ous, though) }\end{array}$ & & \\
\hline \multicolumn{5}{|l|}{ co-meronymy } \\
\hline \multicolumn{5}{|l|}{ Collocation } \\
\hline technical & $\begin{array}{l}\text { - coefficient of fric- } \\
\text { tion, scalar value } \\
\text { - N, R }\end{array}$ & $\begin{array}{l}\text { static friction force, } \\
\text { coefficient of friction } \\
\text { - } f \mathrm{~S}=\mu \mathrm{sN}, \mathrm{N}, \mu\end{array}$ & & \\
\hline non-technical & $\begin{array}{l}\text { - tyre:road } \\
\text { - piston:cylinder }\end{array}$ & - magnitude:force & & $\begin{array}{l}\text { - speed:motion } \\
\text { - smoother:lubrication }\end{array}$ \\
\hline
\end{tabular}


Lexical relations analysis: Hibbeler: Friction

\begin{tabular}{|c|c|c|c|}
\hline Taxonomic & Paragraph 1 & Paragraph 2 & Paragraph 3 \\
\hline \multicolumn{4}{|l|}{ Classification } \\
\hline \multicolumn{4}{|l|}{ co-hyponomy } \\
\hline $\begin{array}{l}\text { class/sub-class } \\
\text { (general- } \\
\text { specific OR } \\
\text { kinds of) }\end{array}$ & $\begin{array}{l}\text { - force of resis- } \\
\text { tance: } \\
\text { - friction }\end{array}$ & $\begin{array}{l}\text { - friction: } \\
\text { - dry friction } \\
\text { - surface: } \\
\text { - contacting, floor }\end{array}$ & \\
\hline contrast & & $\begin{array}{l}\text { - left:right } \\
\text { - rigid:nonrigid }\end{array}$ & \\
\hline synonymy & - prevent:retard & $\begin{array}{l}\text { nonrigid:deformable } \\
\text { - uniform weight:W } \\
\text { - normal force: } \Delta \mathrm{Nn} \\
\text { - frictional force: } \Delta \mathrm{Fn} \\
\text { - applied force:P } \\
\text { - reactive force: } \Delta \mathrm{Rn} \\
\text { - irregularities:protube } \\
\text { rances }\end{array}$ & $\begin{array}{l}\text { - limiting static frictional force:Fs } \\
\text { - normal force: } N \\
\text { - resultant:R } \\
\text { - angle: Øs } \\
\text { - constant of proportionality:u, coefficient of } \\
\text { static friction }\end{array}$ \\
\hline repetition & $\begin{array}{l}\text { - body } \times 5 \\
\text { - force } \times 2 \\
\text { - points } \times 2 \\
\text { - surface } \times 2 \\
\text { - relative } \times 2\end{array}$ & $\begin{array}{l}\text { - friction } \times 6 \\
\text { - block } \times 6 \\
\text { - surface } \times 5 \\
\text { - force } \times 9\end{array}$ & $\begin{array}{l}\text { - force } \times 4 \\
\text { - static friction } \times 3\end{array}$ \\
\hline \multicolumn{4}{|l|}{ Composition } \\
\hline $\begin{array}{l}\text { meronymy } \\
\text { (parts of) }\end{array}$ & & $\begin{array}{l}\text { - frictional forces: } \\
\text { effects of mechanics, } \\
\text { temperature, density, } \\
\text { cleanliness, atomic } \\
\text { attraction }\end{array}$ & - resultant: normal force, frictional force \\
\hline \multicolumn{4}{|l|}{ co-meronymy } \\
\hline \multicolumn{4}{|l|}{ Collocation } \\
\hline technical & - tangent & $\begin{array}{l}\text { nonrigid, deform- } \\
\text { able, distribution, } \\
\text { free-body diagram }\end{array}$ & $\begin{array}{l}\text { - limiting static frictional force, constant of pro- } \\
\text { portionality, coefficient of static friction, angle } \\
\text { of static friction } \\
\text { - Fs }=\mu \mathrm{sN}, \mu \mathrm{s}, \mathrm{N}, \mathrm{Fs}, \mathrm{R}\end{array}$ \\
\hline non-technical & & - equilibrium:balance & \\
\hline
\end{tabular}




\section{Appendix H}

\section{Selected Intersemiotic Analyses of Intermodal Identification}

Intermodal identification analysis: Garnet, by clause, paragraph 1

\begin{tabular}{|l|l|l|l|}
\hline Clause & Rank & \multicolumn{1}{|c|}{ Type of intermodal identification } & \multicolumn{1}{|c|}{ Clause } \\
\hline 1 & element & $\begin{array}{l}\text { intensive: images show shape \& texture of } \\
\text { objects } \\
\text { possessive: images show additional parts of } \\
\text { objects } \\
\text { circumstantial: image show location of parts of } \\
\text { object }\end{array}$ & When two objects are in contact \\
\hline 2 & & & and one object is moved past the other, \\
\hline 3 & & & a force is created \\
\hline 4 & & & $\begin{array}{l}\text { which resists the movement of the ob- } \\
\text { jects: }\end{array}$ \\
\hline 5 & element & $\begin{array}{l}\text { possessive: image visualizes parts of a ma- } \\
\text { chine }\end{array}$ & Friction occurs in all machines \\
\hline 6 & & & it is called friction. \\
\hline 7 & & & $\begin{array}{l}\text { and is usually considered detrimental to } \\
\text { the machines' efficiency, }\end{array}$ \\
\hline 10 & element & $\begin{array}{l}\text { intensive: image shows the nature of the mate- } \\
\text { rials (steel as hard and shiny, etc) }\end{array}$ & $\begin{array}{l}\text { Although the effect that friction has on an } \\
\text { engineering system depends on the nature } \\
\text { of the materials that are in contact, }\end{array}$ \\
\hline 9 & & $\begin{array}{l}\text { but there are times } \\
\text { certain facts are known }\end{array}$ \\
\hline 11 & & to be consistent for all materials. \\
\hline 10 & & & \\
\hline
\end{tabular}


Intermodal identification analysis: Hibbeler: Frictioin, by clause, paragraph 2

\begin{tabular}{|c|c|c|c|}
\hline Clause & Rank & Type of intermodal identification & Clause \\
\hline 1 & & & $\begin{array}{l}\text { The theory of dry friction is best ex- } \\
\text { plained }\end{array}$ \\
\hline 2 & & & by considering the effects \\
\hline 3 & $\begin{array}{l}\text { configu- } \\
\text { ration }\end{array}$ & $\begin{array}{l}\text { language gloss: pulling horizontally identifies } \\
\text { the visual's arrow / uniform weight glosses the } \\
W \text { in the text and in the visual } \\
\text { manner: image of arrow shows that pulling is } \\
\text { directed from the middle of the block, not from } \\
\text { the top or bottom }\end{array}$ & $\begin{array}{l}\text { caused by pulling horizontally on a block } \\
\text { of uniform weight } W\end{array}$ \\
\hline 4 & element & $\begin{array}{l}\text { intensive: image shows texture of verbal rough } \\
\text { surface } \\
\text { language gloss: the visual's label rough surface } \\
\text { identifies the located participant }\end{array}$ & $\begin{array}{l}\text { which is resting on a rough horizontal } \\
\text { surface, Fig. 8-1 a. }\end{array}$ \\
\hline 5 & & & $\begin{array}{l}\text { To properly develop a full understanding } \\
\text { of the nature of friction, }\end{array}$ \\
\hline 6 & & & it is necessary to consider \\
\hline 7 & & & $\begin{array}{l}\text { the surfaces of contact to be nonrigid or } \\
\text { deformable. }\end{array}$ \\
\hline 8 & & & $\begin{array}{l}\text { The upper portion of the block, however, } \\
\text { can be considered rigid. }\end{array}$ \\
\hline 9 & & $\begin{array}{l}\text { language gloss: identifies the visual, Fig. 8-1b, } \\
\text { as a free-body diagram }\end{array}$ & $\begin{array}{l}\text { As shown on the free-body diagram of } \\
\text { the block, Fig. 8-1b, }\end{array}$ \\
\hline 10 & $\begin{array}{l}\text { configu- } \\
\text { ration }\end{array}$ & $\begin{array}{l}\text { manner: the repeated arrows show the direction } \\
\text { of the exertion, that the exertion is even, the } \\
\text { extent of the distribution, its continuity }\end{array}$ & $\begin{array}{l}\text { the floor exerts a distribution of both } \\
\text { normal force } \Delta \mathrm{Nn} \text { and frictional force } \\
\Delta \mathrm{Fn} \text { along the contacting surface. }\end{array}$ \\
\hline 11 & $\begin{array}{l}\text { configu- } \\
\text { ration }\end{array}$ & \multirow{2}{*}{$\begin{array}{l}\text { Cause: result: image of upward arrows and } \\
\text { downward arrows shows the result of the lan- } \\
\text { guage, which is the block not falling or tipping }\end{array}$} & $\begin{array}{l}\text { For equilibrium, the normal forces must } \\
\text { act upward, }\end{array}$ \\
\hline 12 & & & to balance the block's weight $\mathrm{W}$ \\
\hline 13 & $\begin{array}{l}\text { configu- } \\
\text { ration }\end{array}$ & \multirow{3}{*}{$\begin{array}{l}\text { Cause: result: image of left arrows and right } \\
\text { arrows shows the result of the language, which } \\
\text { is the block not falling or tipping (can see that } \\
\text { arrows counterbalance one another) }\end{array}$} & and the frictional forces act to the left \\
\hline 14 & & & to prevent the applied force $P$ \\
\hline 15 & & & from moving the block to the right. \\
\hline 16 & $\begin{array}{l}\text { configu- } \\
\text { ration }\end{array}$ & \multirow{2}{*}{$\begin{array}{l}\text { Causal: reason/condition: visual shows reason } \\
\text { for development of the } 2 \text { forces is the bumpi- } \\
\text { ness and contact }\end{array}$} & $\begin{array}{l}\text { Close examination of the contacting sur- } \\
\text { faces between the floor and block reveals }\end{array}$ \\
\hline 17 & & & $\begin{array}{l}\text { how these frictional and normal forces } \\
\text { develop, Fig. 8-1c. }\end{array}$ \\
\hline
\end{tabular}




\begin{tabular}{|l|l|l|l|}
\hline Clause & Rank & \multicolumn{1}{|c|}{ Type of intermodal identification } & \multicolumn{1}{c|}{ Clause } \\
\hline 18 & element & $\begin{array}{l}\text { intensive: image shows the irregularities' qual- } \\
\text { ity: bumpiness and shape texture }\end{array}$ & It can be seen \\
\cline { 4 - 5 } & & $\begin{array}{l}\text { that many microscopic irregularities exist } \\
\text { between the two surfaces }\end{array}$ \\
\hline 20 & $\begin{array}{l}\text { configu- } \\
\text { ration }\end{array}$ & $\begin{array}{l}\text { Causal: manner: visual shows that reactive } \\
\text { forces develop at same point along surface as } \\
\text { frictional and normal forces }\end{array}$ & $\begin{array}{l}\text { and, as a result, reactive forces } \Delta R n \text { are } \\
\text { developed at each of the protuberances. }\end{array}$ \\
\hline 21 & & & These forces act at all points of contact, \\
\hline 22 & $\begin{array}{l}\text { configu- } \\
\text { ration }\end{array}$ & $\begin{array}{l}\text { Causal: reason: reactive forces contribute to } \\
\text { frictional and normal because they develop in } \\
\text { the same place as frictional and normal forces }\end{array}$ & $\begin{array}{l}\text { and, as shown, each reactive force con- } \\
\text { tributes to both a frictional component } \\
\Delta \text { Fn and a normal component } \Delta N n .\end{array}$ \\
\hline
\end{tabular}

